

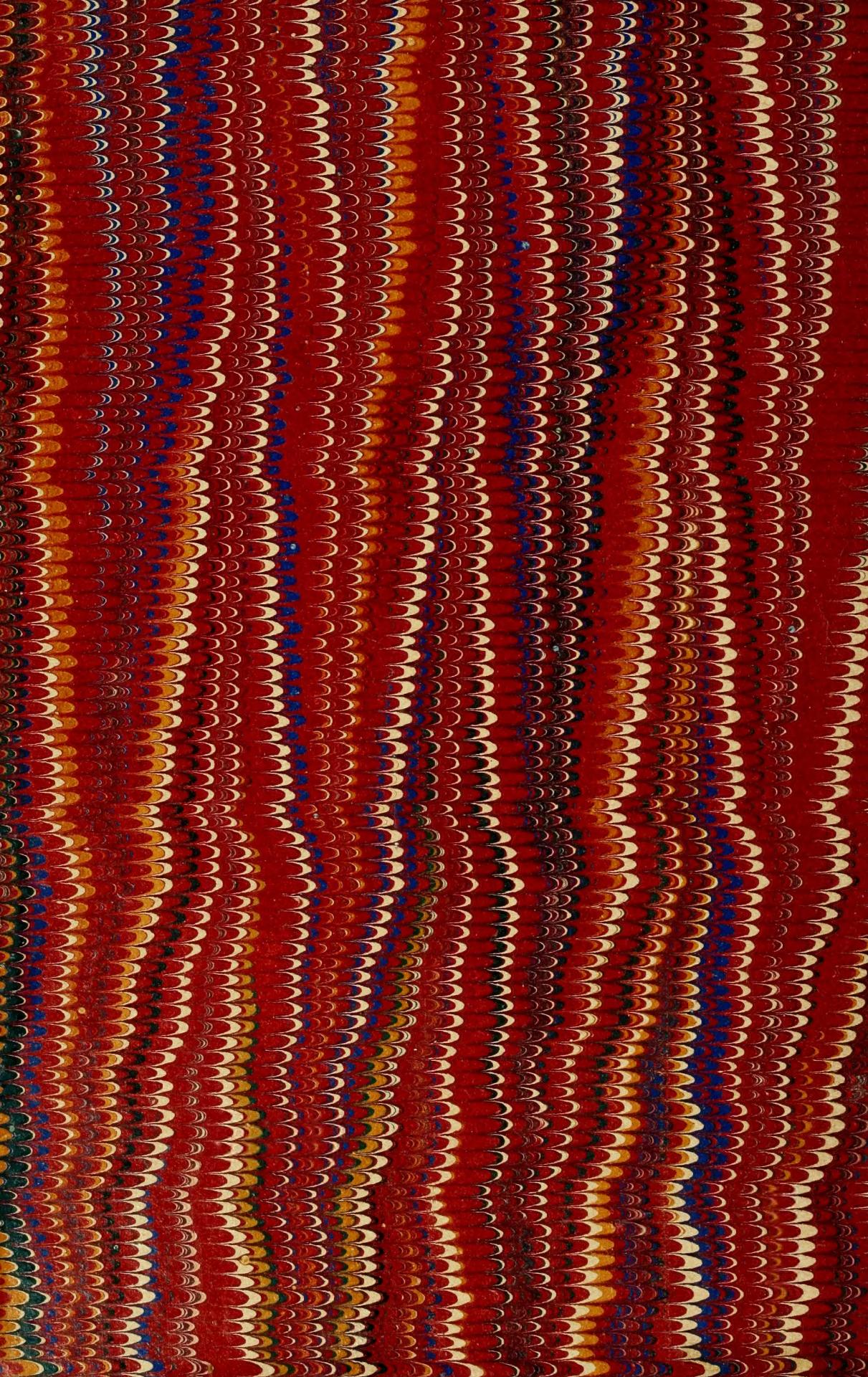




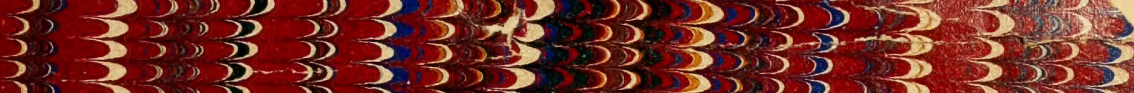

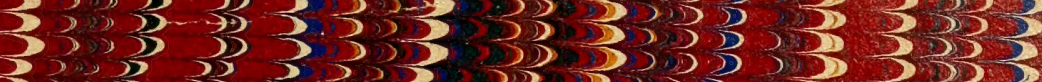

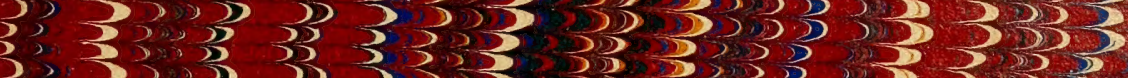

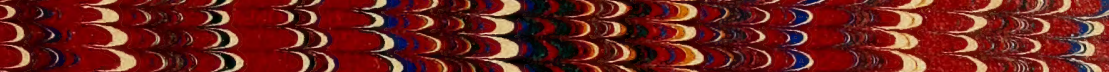

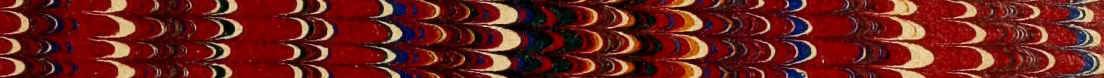

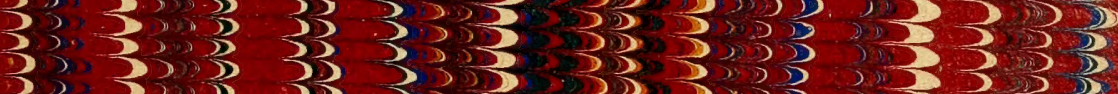

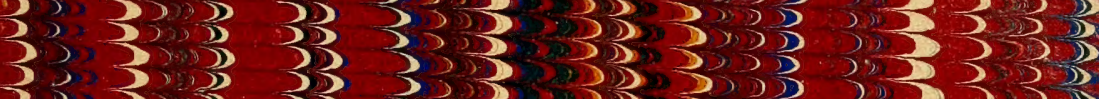

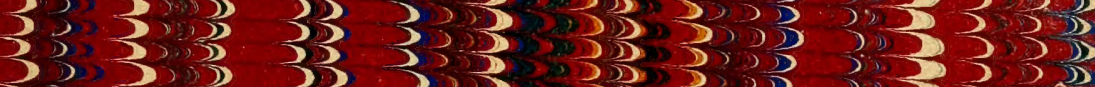

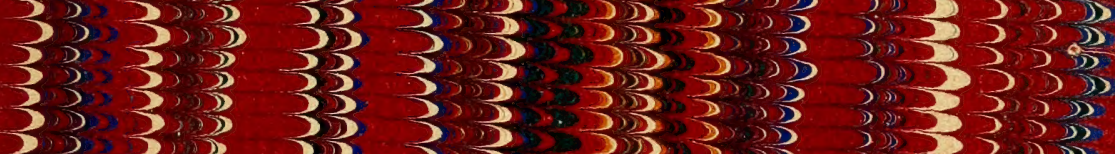

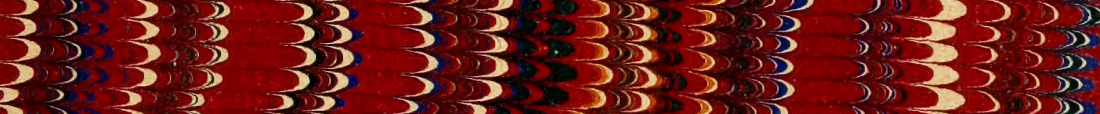

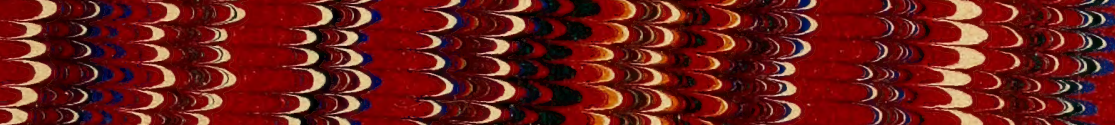

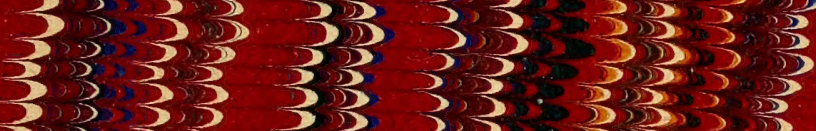

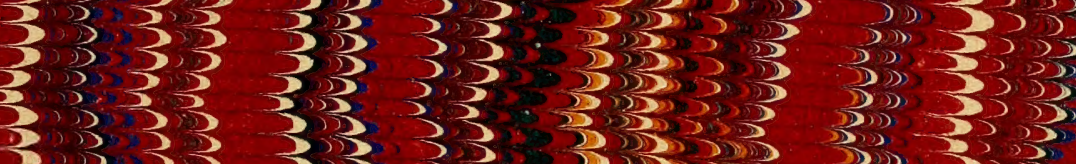

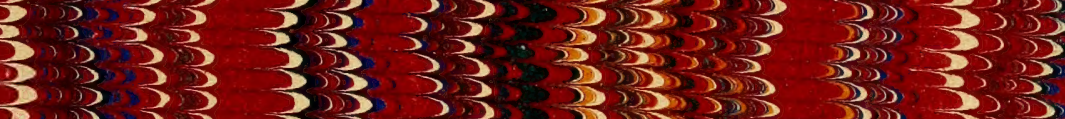

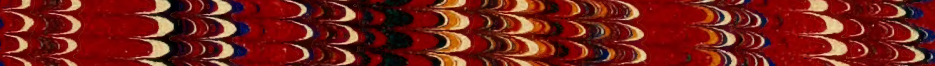

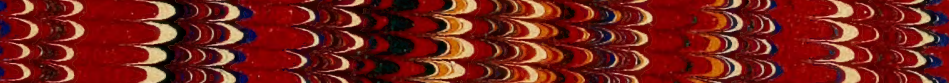

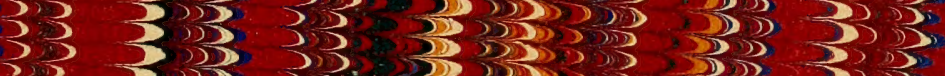

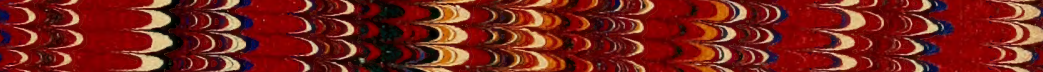

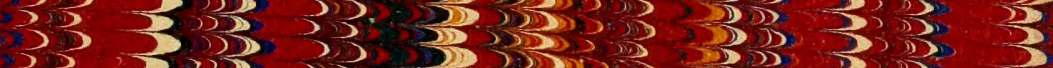

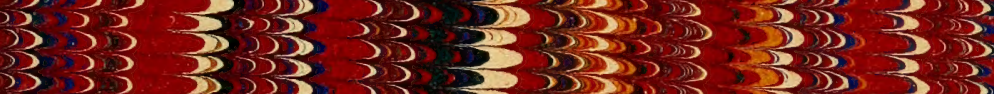

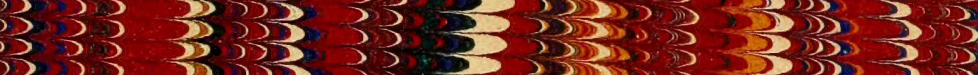

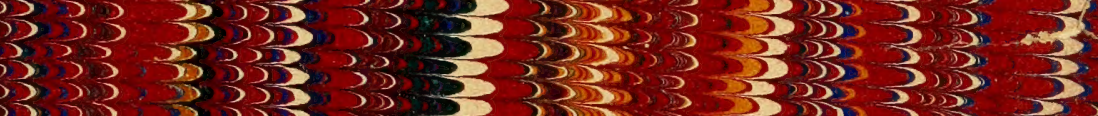

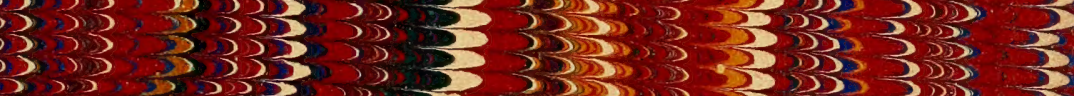

$3>5,3035$

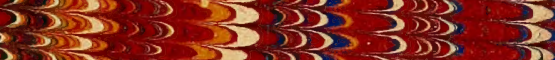

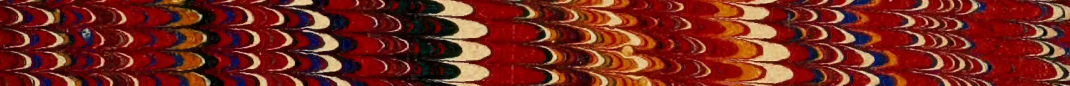

353333

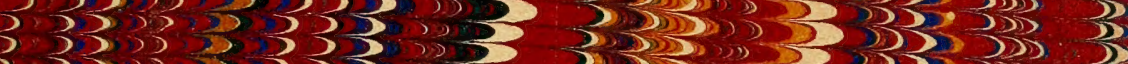

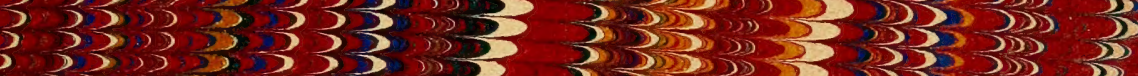

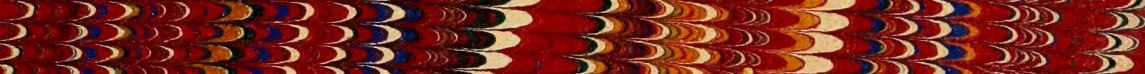

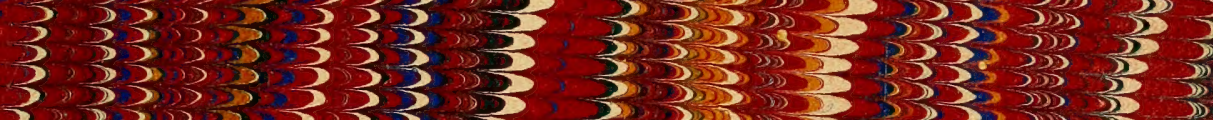

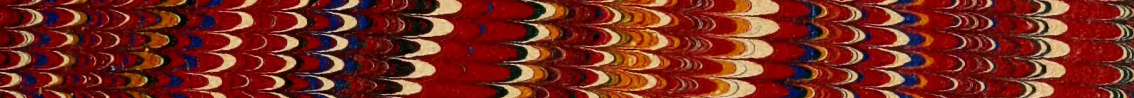

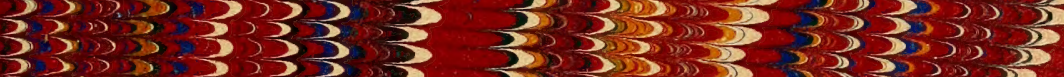

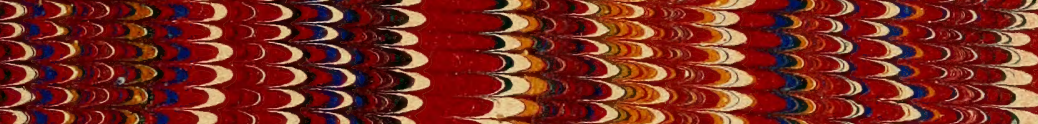

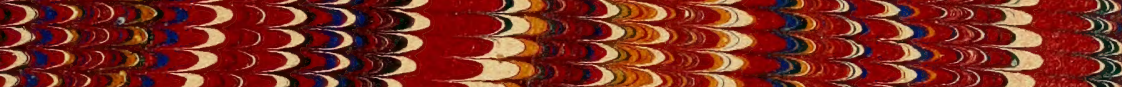

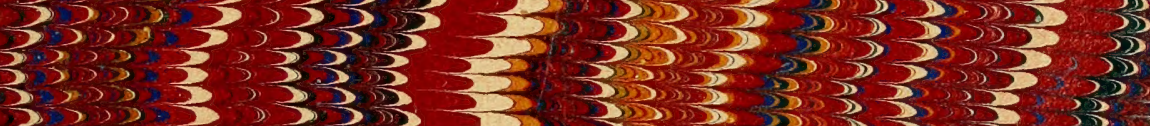

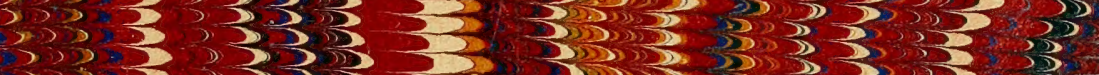

$3,3,3,33 \pi 35,35,353$

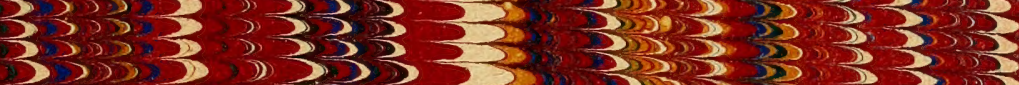

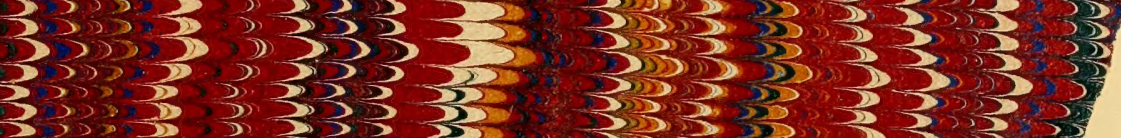




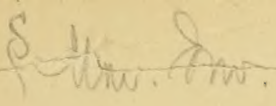

I

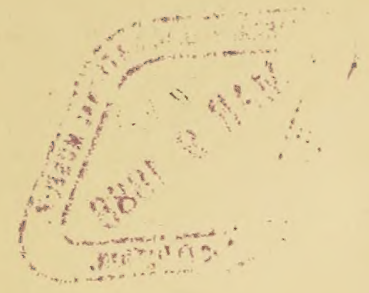



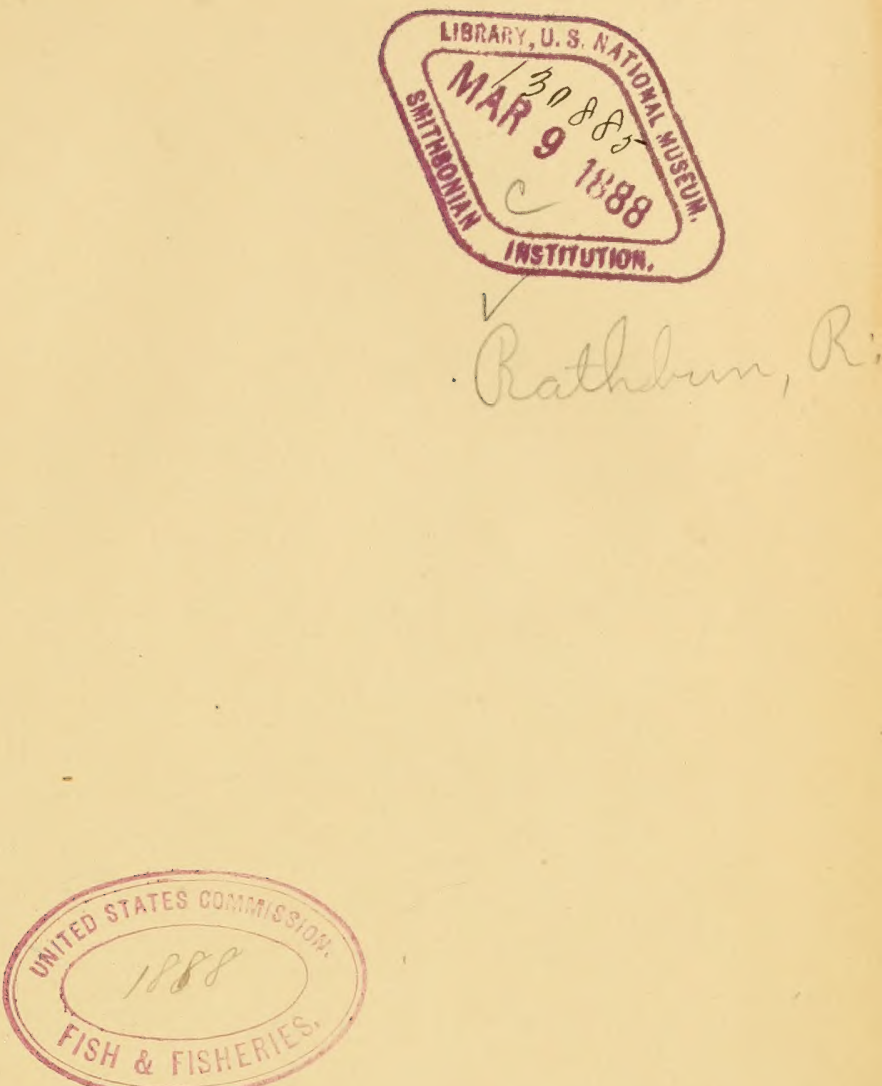




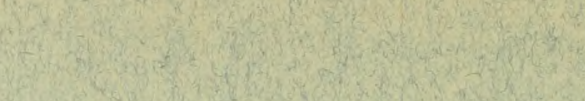

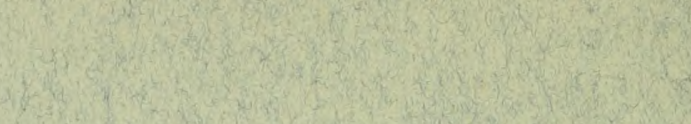


OF THE

\section{DREDGING STATIONS}

or $\operatorname{mix}$

UNITED STATES FISH COMYISSION

FROM

1871 TO 1879 INCLUSIVE,

WIIH

TEMPERATURE AND OTHER OBSERVATIONS.

[ ARRANGED FOR PUBLICATION BY SANDERSON SMITH AND RICHARD RATHBUN.]

[EXTRACTED FROM THE ANNUAL REPORT OF THE COMMISSIONER OF FISH AND FISHERIES FOR 1879.]

WASHINGTON:

GOVERNMENT PRINTING OFFICE.

1882 . 

000.-LISTS OF THE DREDGING STATIONS OF THE UNITED STATES FISH COMINISSION FROM 1871 TO 1879, INCLUSIVE, WITH TEMPERATURE AND OTHER OBSERVATIONS.

[Arranged for publication by SANDERson Surtu and Richard RATHuUN.]

The following lists include all the recorded dredging stations made by, or in connection with, the United States Fish Commission, from its organization up to date. The stations are, for the nost part, arranged chronologically, and are designated by four series of numbers or letters, as follows: One series of numbers, from 1 to 87 , with letters appended, represents the stations for 1871 . The 1872 stations (in the Bay of Fundy) are designated by letters from $t$ to $z$. Those for 1873 are indicated by a second series of numbers, from 1 to 212, with B. (Bache) or Bl. (Bluelight) added, according as the dredgings were carried on from the steamers Bache or Bluelight. In this series, however, are also included the stations of the Bache for 1872 and 187t, as well as those for 1873. The last series combines all the stations from 1874 to 1879 , inclusice (omitting 1876 , during which year sea-rork was suspended), in numbers rumning from 1 to 769 . For the sake of obtaining greater uniformity in recording the stations on charts, as explained further on, the stations for 1874 and 1875 , originally numbered separately, have been united with those from 1877 to 1879 , and giren numbers following 1879 . The numbers for these later years rum as follows: 187 , from 400 to $580 ; 1875$, from 600 to 769 ; 1877 , from 1 to $128 ; 1878$, from 129 to 238 ; 1879 , from 239 to 378 .

A chart has been prepared by $\mathrm{MI}$. Smith to illustrate the dredgings of the Commission north of Cape Cod, exclusive of those of 1572 in the Bay of Fundy; those of the Bache, on the Banks, in 1872; and of the Speedwell, off the coast of Nova Scotia, in 1877. On this chart the stations of the speedwell for 1877, 1879, and 1879 are indicated by numbers only, and are readily distinguished from those of the Bache and Bluelight, which have B. or Bl. afixed to them. In such of the following atables as refer to this chart, the localities given are taken from the original record books, whenerer such exist (i.e., for all the work of the Speedwell and much of that of the Bluelight-101 Bl. to $166 \mathrm{BI}$.), with some other notes added to facilitate the finding of the localities on the chart. In many eases the positions were marked, at the time, on the steamers' charts by the commanding officer, and all such positions have been adopted, even though diflering somewhat from those giren by the record looks. From the nature of the operations of dredging and trawling, it becomes almost impossible to estimate exactly the changes of position cansed by emrents, sce, especially when out of sight of land, and in at 
few cases the positions were not placed on the charts at the time, and the bearings given do not suffice to fix them very accurately. It is believed, however, that but few positions laid down on the chart are rendered uncertain to any great extent by either of these causes. A large part 0 :" the positions determined by the Bache were originally given by latitude and longitude. The other latitudes and longitudes given in the tables are taken from the accompanying chart, and are intended to serve as the readiest means of finding the localities, all of which are either thus designated or are referred to as being near others, which are so. Of the dredging stations north of Cape Cod, Nos. 79 B to 97 B, 37 to 123 are outside of the limits of the chart. These, and all others of the northern stations, not placed upon the chart, are marked with + before the serial number. The bearings given for the Speedwell's work in 1879 are true; the others, with a few (unrecognizable) exceptions, are magnetic.

In the last column of the tables the letter indicates the apparatus employed in dredging: D., Dredge; Ag. D., Agassiz Dredge; R. D., Rake Dredge; T., Trawl; Ag. T., Agassiz Trawl; O. T., Otter Trawl; Tan., Tangles.

STATIONS FOR 1871, IN AND ABOUT VINEYARD SOUND, MASSACHUSET'T'S.

During this, the first year of the Commission, the dredgings in shallow water were made partly from a sail-boat, and partly from a steamlaunch, and those in the deeper waters, from the United States revenuecutter Moceasin, Capt. J. G. Baker. The dredging stations numbered in all about 250 , but to avoid confusion in laying them out on the chart, they were combined into 87 groups or lines, each including from 2 to 9 statious, the lines being designated by numbers, the stations by letters. In this mamer they were represented on the large chart accompanying the Report of the United States Fish Commissioner for 1871-72. In making up the present list the same arrangement has also been followed, and where all the stations of a group were of the same nature, they hare been located collectively; otherwise the exact position of each station has been given.

Dates are not prefixed to all of the inner groups, as many of these include stations made on different days. Temperature observations (with Miller-Casella self-registering thermometers) were taken at most of the outer stations, as recorded in the list, but were omitted at the inner ones. The dredge was the implement most commonly used for scraping the bottom, but the beam-trawl was also frequently emplojed on the smooth inner grounds. The rake-dredge was worked a fer times off Gay Head, and the tangles very rarely, in only a few places. The characters of the many localities gone over in 1871, as well as the species of animals found inhabiting them, are fully discussed in the "Report upon the Invertebrate Animals of Vineyard Sound and the adjacent waters, with an acconnt of the physical characters of the region," by Prof. A. E. Verrill; contained in the Report of the United States Fish Commissioner, Part I, for 1871-'72. 


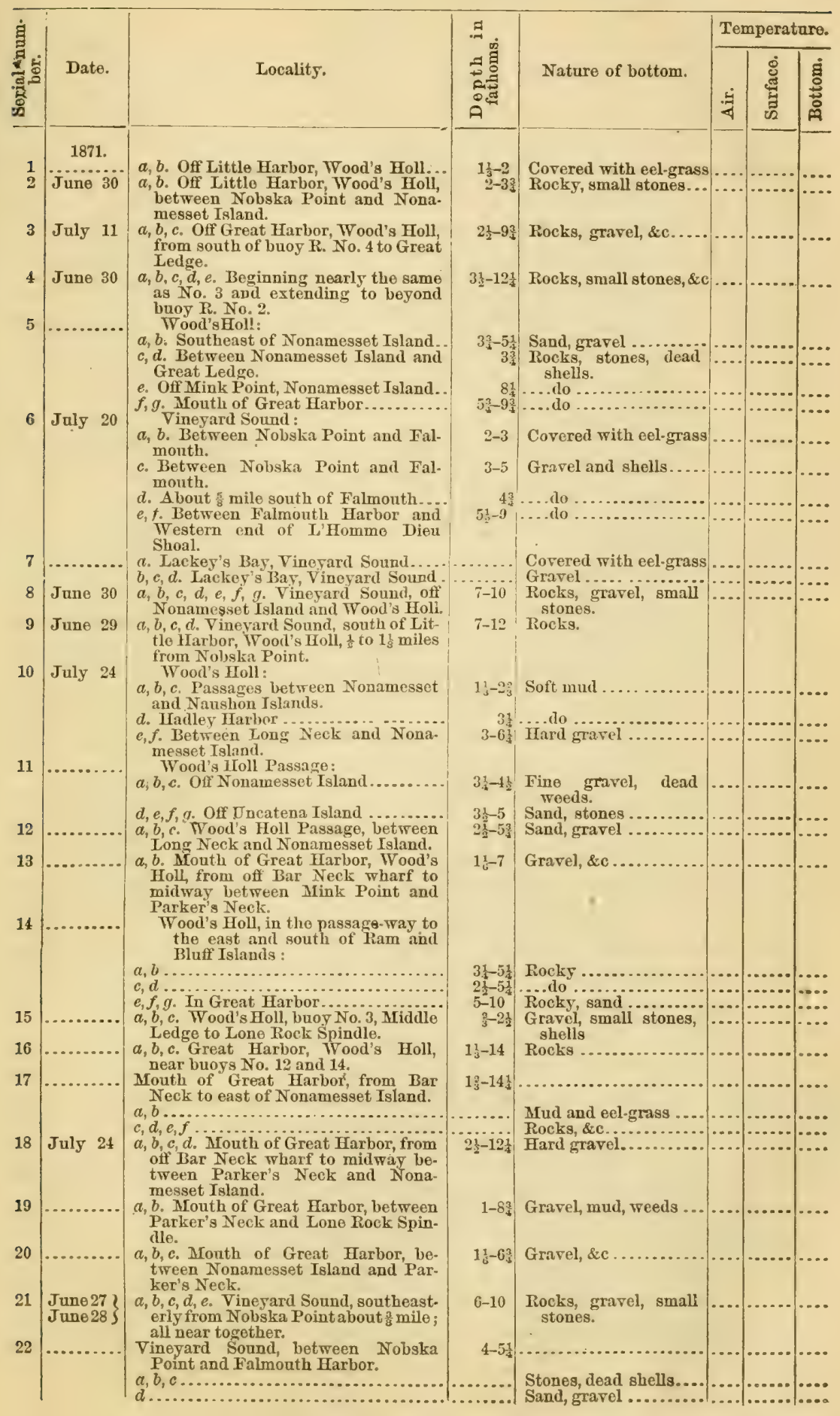




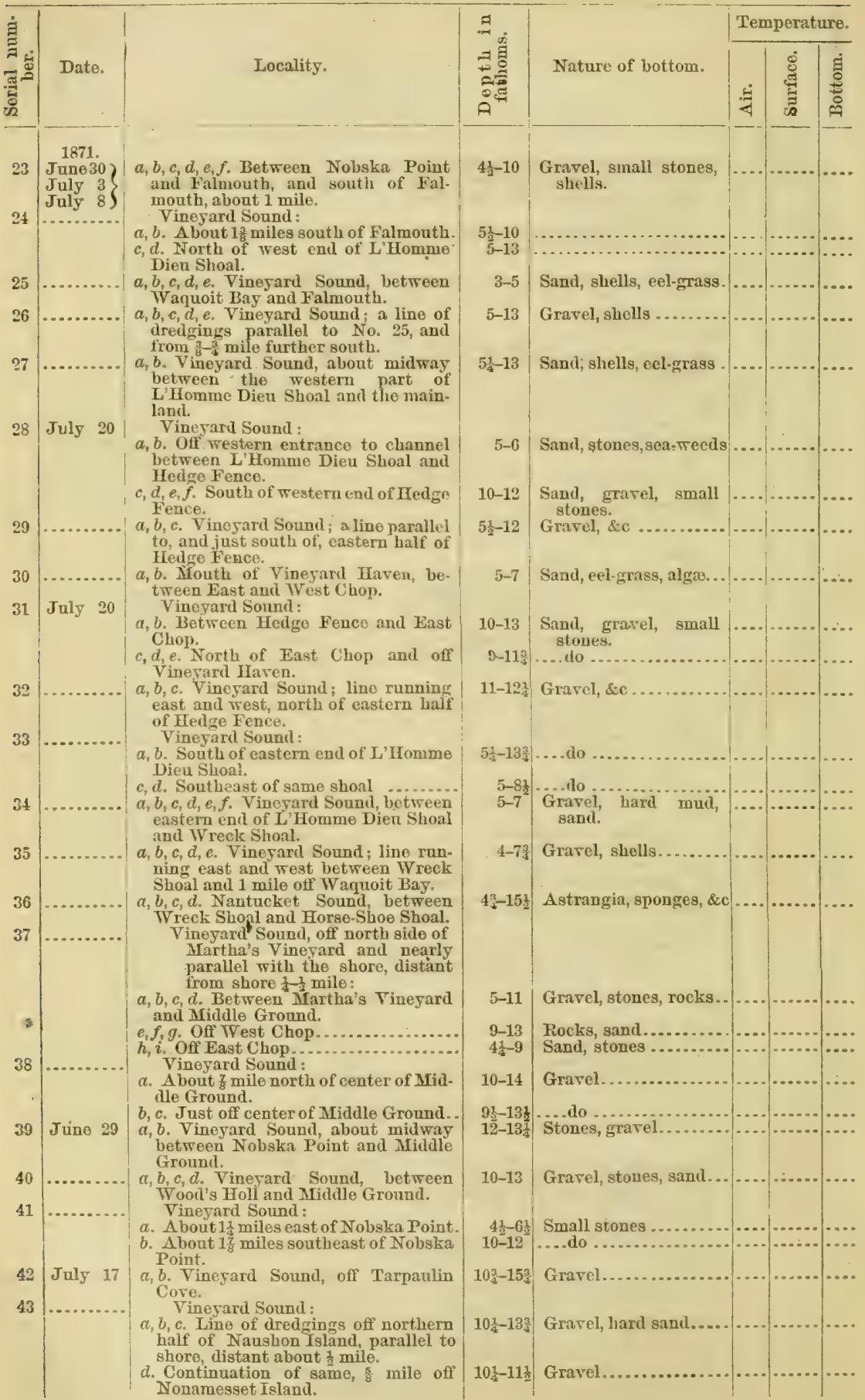




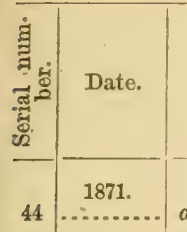

45

July 24
Locality.

$a, b, c, d, e$. Vineyard Sound; line about parallel to last, off northern half of Naushon Island, about 1 milo from shore.

$a$. b. Vinesard Sound, off Quick's Hole. Vineyard Sound, off the Elizabeth Islands.

$a, b$. Off Pasque Island

c. Off Robinson's Hole

$d, e$. Off south end of Naushon Island $a, b, c, d, e$. Vineyard Sound, off west side of Martha's Vineyard, between Menemsha Bight and Cedar Tree Neck, $\frac{1}{2}$ to $1 \frac{1}{4}$ miles from shore, and nearly parallel to it.

$a, b, c, d$

e.

$a, b, c, d$. Vineyard sound, same as last $\frac{1}{4}-\frac{1}{2}$ mile from shore, and extending about $1 \frac{1}{2}$ miles both north and south of Cape Higgon.

Vineyard Sound:

a. About northeast of Gay Head, $4 \frac{1}{6}$ miles.

b. About west of Lucas Shoal, $1 \frac{1}{8}$ miles

$a, b$. Vineyard Sound, Menemsha Bight Vineyard Sound, Menemsha Bight:

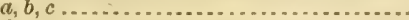
d.

$e$

July 14$\} \quad a, b, c, d$. Vineyard Sound, north of July 17$\}$ southwestern extremity of Martha's Vineyard, $\frac{1}{2}-1$ mile from shore $(c, d$, oft' Menemsha IBight).

July $14 \mid a, b, c, d, e$. Vineyard Sound, north and northeast of Deril's Bridge, Gay Head, $\frac{3}{8}$ to 1 mile from shore.

Vineyard Sound, nortl of Gay Head:

a. About $1 \frac{1}{2}$ miles from shore

b. About 2t miles from shore

July $22 \mid a, b, c$. Vineyard Sound, north of Devil's Bridge, Gay Head, $\frac{3}{4}$ mile.

July $22 a, b, c, d$. Vinerard Sound, northwest erly from Gay Head, about 1 mile.

$a, b, c, d, e$. About same position as last, forming a line about $\frac{1}{5}$ mile further off.

$a, b, c, d, e$. Vineyard Sound, northwesterly from Gay Head, $13-2$ miles.

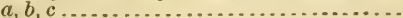

$a, b, c$. Vineyard Sound, northwesteriy from Gay Head, $\frac{1}{2}-15$ miles.

$a, b, c$. Vineyard Sound, northwesterly from Gay Head, 1-2 miles.

$a, b, c$. Vineyard Sound, northwesterly from Gay Head, 1-2 miles; more easterly than 60 .

$a, b, c$, Vineyard Sound, northwesterly from Gay Head, 1-2 miles; more easterly than 61 .

$a, b, c, d$. Vineyard Sound, north of Devil's Bridge, Gay Head, $\frac{1}{8}-\frac{1}{4}$ mile.

July 12 Buzzard's Bay, Cataumet Harbor: a. In harbor

b. At mouth of harbor

$a, b$. Buzzard's Bay, off Cataumet Har. bor.

$a, b$. Buzzard's Bay, between Long Neck and Quamquisset Harbor. $a, b$. Just outside of 66 .

Buzzard'sBay, west of Quamquisset Harbor:

a. About 2 miles

b. A About 1 mile

c. About 3 mile

$a, b$. Brzzard's Bay, west of Quamquis.

set Harbor, about $1 \frac{1}{2}$ miles.

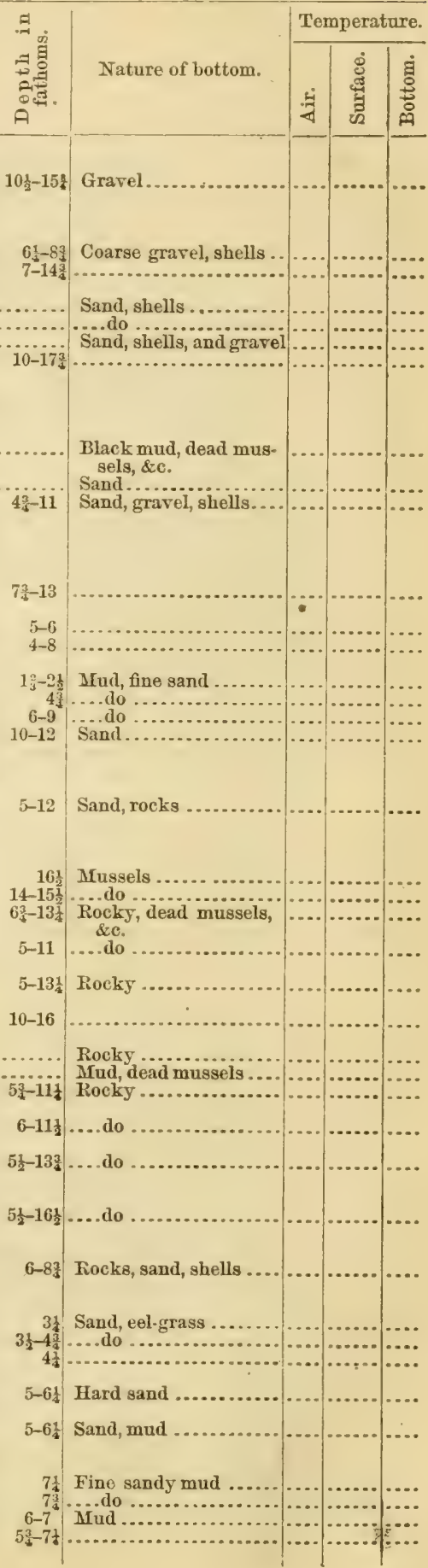




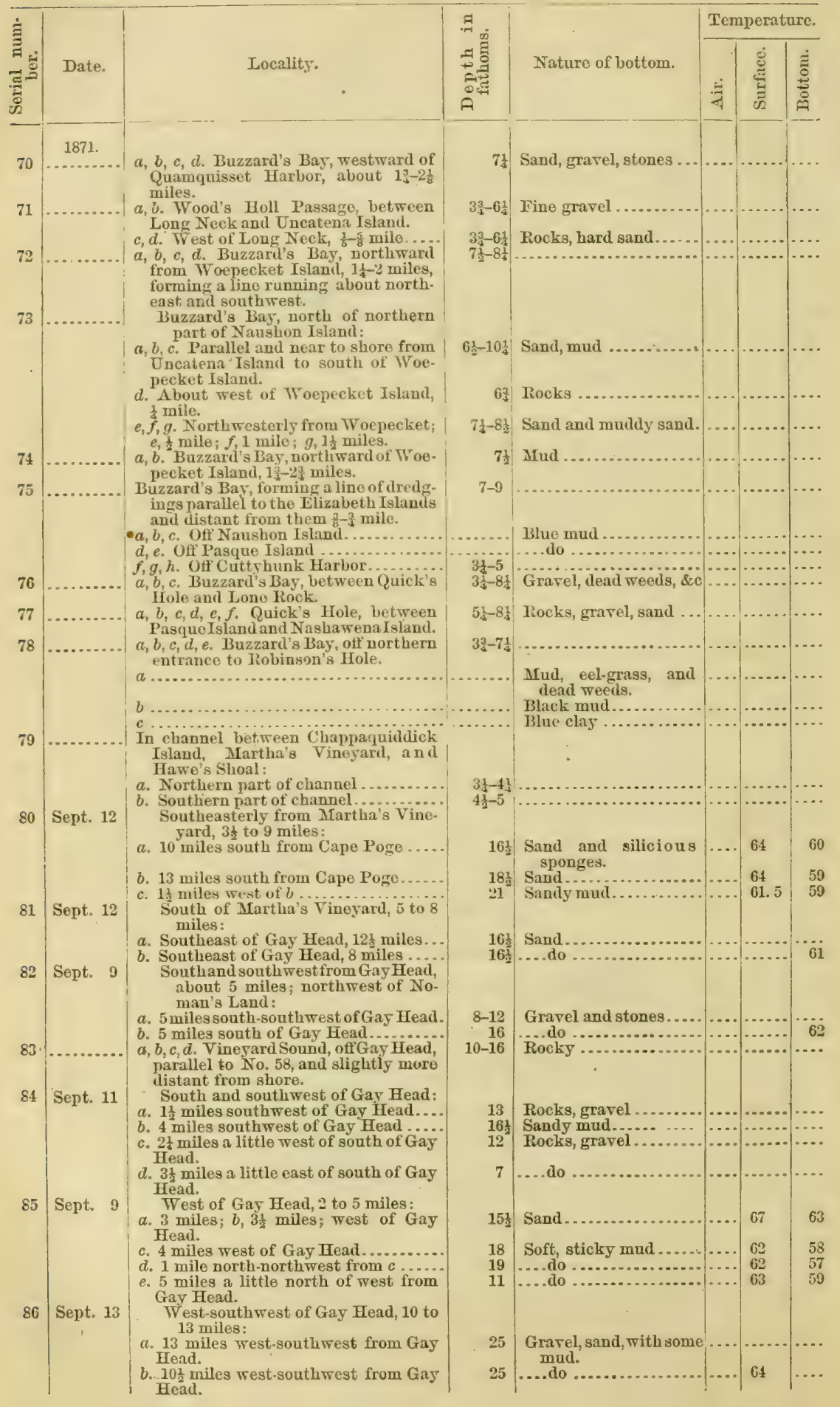




\begin{tabular}{|c|c|c|c|c|c|c|c|c|}
\hline \multirow{2}{*}{ 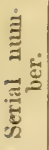 } & \multirow[b]{2}{*}{ Date. } & \multirow{2}{*}{\multicolumn{2}{|c|}{ Locality. }} & \multirow{2}{*}{ 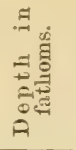 } & \multirow[b]{2}{*}{ Nature of bottom. } & \multicolumn{3}{|c|}{ Temperature. } \\
\hline & & & & & & $\ddot{z}$ & 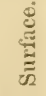 & 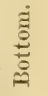 \\
\hline 87 & $\begin{array}{l}1871 . \\
\text { Sept. } 14\end{array}$ & $\begin{array}{l}\text { a. } 19 \frac{1}{2} \text { miles } \\
\text { Head. } \\
\text { b. } 18 \frac{1}{2} \text { miles } \\
\text { Head. }\end{array}$ & $\begin{array}{l}\text { west-southwest of Gar } \\
\text { west-southrest of Gay }\end{array}$ & $\begin{array}{l}29 \\
29\end{array}$ & $\begin{array}{l}\text { Sandy mud........ } \\
\text {....do ............... }\end{array}$ & $\ldots$ & 62 & $\begin{array}{r}\cdots \\
59\end{array}$ \\
\hline
\end{tabular}

STATIONS FOR 1872, WITII HEADQUARTERS AT EASTPORT, ME.

The dredgings for 1872 were mostly carried on from a large sail-boat; but those in the deeper waters of the Bay of Fundy were made from the United States revenue-cutter Mosswood, Captain Hodgdon. The regions explored were about as follows: Eastport Harbor, South Bay, and Passamaquoddy Bay, all of which are comparatively shallow-water areas; the sliallow waters about the island of Grand Menau, especially those among the small islands to the east of Grand Menan; and the deeper waters east of Campobello Island, west of Grand IIenan; and toward the center of the Bay of Fundy, between Grand Menan and Nova Scotia.

In connection with the shallow-water dredgings no complete record of observations was kept, but the collections made were appropriately labeled with the precise locality, depth of water, nature of bottom, \&c. The more important hauls in deep rater, mostly accompanied by temperature observations, are as follows, the letters used to designate them being the same as were employed in the original records:

\begin{tabular}{|c|c|c|c|c|c|c|}
\hline \multirow{2}{*}{ 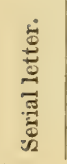 } & \multirow[b]{2}{*}{ Date. } & \multirow[b]{2}{*}{ Locality. } & \multirow{2}{*}{ 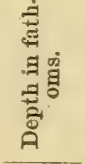 } & \multicolumn{3}{|c|}{ Temperatures. } \\
\hline & & & & $\ddot{\nexists j}$ & 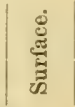 & 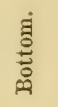 \\
\hline$+t$ & $\begin{array}{l}\text { 1872. } \\
\text { Ang. } 24\end{array}$ & $\begin{array}{l}\text { Bay of Fundy, off Grand Menan, beginning at a point } 8 \\
\text { miles SE. by E. of north end of White Head Island, and } \\
\text { running NE. for a distance of nearly } 3 \text { miles. (Tem- } \\
\text { peratures taken at the beginning and close, and the same } \\
\text { at both.) }\end{array}$ & $106-30$ & $\begin{array}{c}0 \\
\cdots \cdots\end{array}$ & $\circ 8$ & $\stackrel{\circ}{38}$ \\
\hline$+t^{\prime}$ & ... do ... & $\begin{array}{l}\text { Bay of Fundy, off Grand Menan, north of last; beginning } \\
8 \frac{1}{3} \text { miles } \mathbf{E} \text {. of White Head Island, and extending about } \\
2 \text { miles SW. }\end{array}$ & $96-100$ & & ........ & $37 \frac{3}{4}$ \\
\hline$+u$ & Aug. 23 & $\begin{array}{l}\text { Bay of Fundy, E. of Grand Menan, about } 2 \frac{1}{2} \text { miles E. of } \\
\text { north end of White Head Island. }\end{array}$ & $28-52$ & & 53 & 391 \\
\hline$u u^{\prime}$ & ... do & $\begin{array}{l}\text { Bay of Fundy, E. of Grand Menan, } 15 \text { miles E. by S. } \frac{3}{4} \text { S. of } \\
\text { north end of White Head Island. }\end{array}$ & 29 & & ...... & 44 \\
\hline$+v$ & Aug. 28 & $\begin{array}{l}\text { Grand Menan channel, west of Grand Menan Island ; } 2 \frac{2}{3} \\
\text { miles } \mathrm{N} \text {. by W. } \frac{1}{2} \mathrm{~W} \text {. of Southern Head, G. M. }\end{array}$ & 40 & & 48 & $45 \frac{1}{2}$ \\
\hline$+v^{\prime}$ & ... do . & $\begin{array}{l}\text { Grand Menan channel, west of Grand Menan Island; } 4 \frac{1}{4} \\
\text { miles NNW. } \frac{1}{2} \text { W. of Southern Head, G. M. }\end{array}$ & 54 & & 47 & 40 \\
\hline$\therefore v^{\prime \prime}$ & ... do & $\begin{array}{l}\text { Grand Menan channel, west of Grand MI nan Island; } 6 \\
\text { miles N. } \frac{1}{8} \text { WV of Southern Head, G. MI. }\end{array}$ & 55 & & & 40 \\
\hline$+2 v$. & Ang. 16 & $\begin{array}{l}\text { Bay of Fundy, about } 3 \frac{1}{2} \text { miles east of Herring Cove Head, } \\
\text { Campobello Island. (Soft muddy bottom.) }\end{array}$ & 60 & & & 43 \\
\hline$+2 w^{\prime}$ & ... do ... & Bay of Fundy, just off Herring Cove, Campobello Island.. & 27 & & $\cdots$ & 46 \\
\hline$+x$ & Aug. 2 & $\begin{array}{l}\text { Bay of Fundy, } 2 \frac{1}{2} \text { miles, about SE. of Head Harbor Light, } \\
\text { Campolvello Island. }\end{array}$ & 90 & & $48 \frac{1}{2}$ & $\cdot 39 \frac{3}{4}$ \\
\hline$+x^{\prime}$ & ... do & $\begin{array}{l}\text { About } 21 \text { miles ENE. of Heat Harbor Light, Campobello } \\
\text { Island. }\end{array}$ & 77 & & (........ & 42 \\
\hline$+x^{\prime \prime}$ & ... do ... & About $1 \frac{2}{3}$ miles NE. of Head Harbor Light ............... & 30 & & $\ldots$ & 46 \\
\hline $\begin{array}{l}+x^{\prime \prime \prime} \\
+y\end{array}$ & Aug. & $\begin{array}{l}\text { Midway between Head Harbor Light and Spruce Island.. } \\
\text { Passamaguoddy Bay oft' North Harbor. Deer Island.... }\end{array}$ & $\begin{array}{l}73 \\
25\end{array}$ & & 48 & $\begin{array}{l}45 \\
47\end{array}$ \\
\hline T20. & ... do ... & Passamaquoddy Bay, $1 \frac{1}{4}$ miles north of last................ & 32 & & 58 & 46 \\
\hline
\end{tabular}


STATIONS FOR 18\%3, WITI ILEADQUALTERS AT PEAK'S ISLAND, CASCO BAY, MAINE; AND ALSO STATIONS OF THE LNITED STATES COAST SURVEY STEAMER BACHE FOR 1872, 1873, AND 1874, IN THE GULF OF MAINE, \&C.

In this list the dredgings indicated by the above heading have been grouped together, as they appear on the chart prepared for publication. Numbers ranging from $1 \mathrm{~B}$. to $7 \mathrm{~S} \mathrm{B.} \mathrm{were} \mathrm{originally} \mathrm{assigned} \mathrm{to}$ the Bache dredgings for 1873 and 1874, in papers jublished by Professor. Verrill in the American Jourual of Seience for. April, 1874, and June, 1875, and elsewhere. To these the dredging stations of the Bache for 1872,18 in number, have been added, thus increasing the list to $97 \mathrm{~B}$. As to the regular series of dredgings marle by the Bluelight, under command of Lieut. Commander L. A. Beardslee, in and off Casco Bay, no serial numbers were given to the hauls until the commencement of the temperature observations, July 21 . To the numbers ( 1 to 66 ), given to such of the subsequent hauls as were accompanied by temperature observations, 100 has been added (101 L1. to $166 \mathrm{Bl}$.), and numbers from 167 Bl. to 190 Bl. have been given to the hauls from July 12 to July 21, and from 191 Bl. to 212 Bl. to those taken after July 20, but not included in the temperature series. The descriptions of localities from $101 \mathrm{Bl}$. to $166 \mathrm{Bl}$ are taken from the record books for temperatures, with some additions, and from 167 I3l. to $212 \mathrm{Hl}$. from the eight books of dredging lists, which were kept. Additions to $101 \mathrm{Bl}$. to $166 \mathrm{Bl}$., taken from the dredging books, are marked D. L.

The dredging stations of the Bache for 1872 were on and about Saint George's Bank and La Have Bank, and extended as far as Halifax, N. S.; in 1873 they were mostly in the Gulf of Maine, especially in the region of Jeffrey's and Cashe's Ledges, a few being made in Massachusetts Bay; those for 1874 were entirely in the Gulf of Maine. 
"pasn

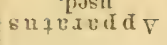

\begin{tabular}{|c|c|c|c|c|c|}
\hline$\stackrel{0}{\Xi}$ & ·тонұод & 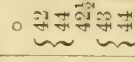 & 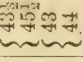 & : & 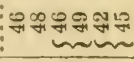 \\
\hline 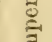 & 'әәвуп & $\circ$ 15 & เै & 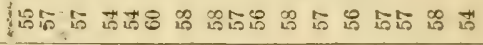 & 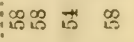 \\
\hline है & $\cdot$ & $\circ$ ○ & 8 & 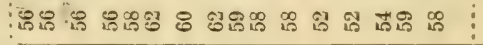 & 约苗 8 \\
\hline
\end{tabular}

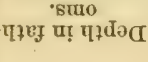

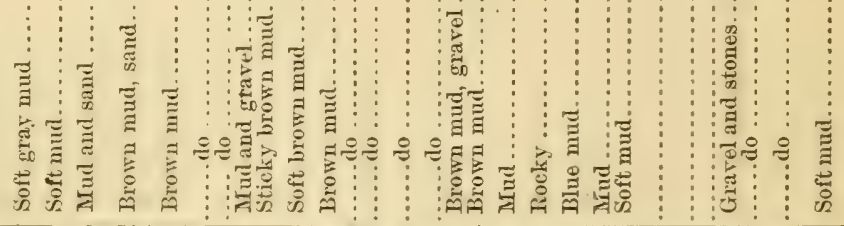

产

긴
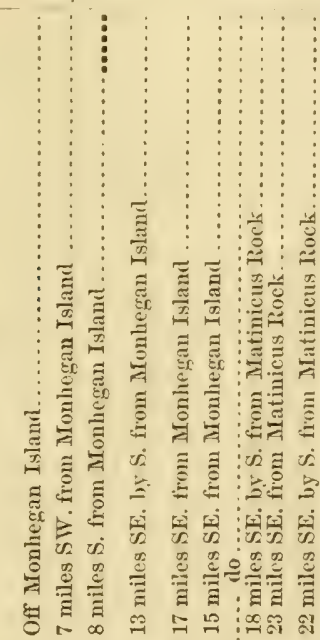

芦

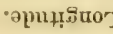

든 늑

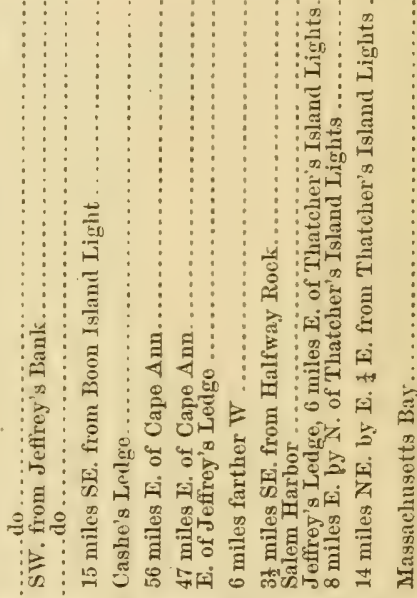

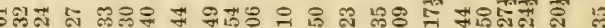

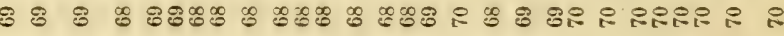

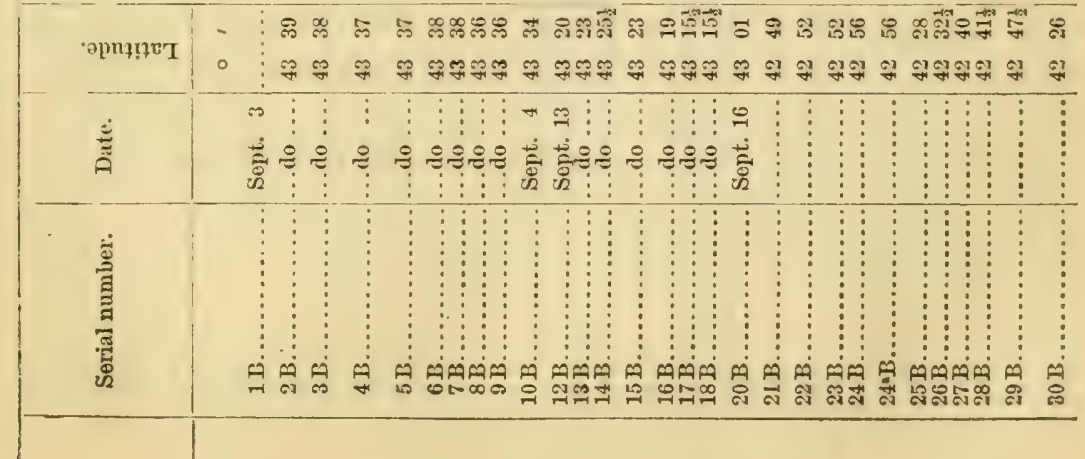


10 REPORT OF COMMISSIONER OF FISH AND z FISHERIES.

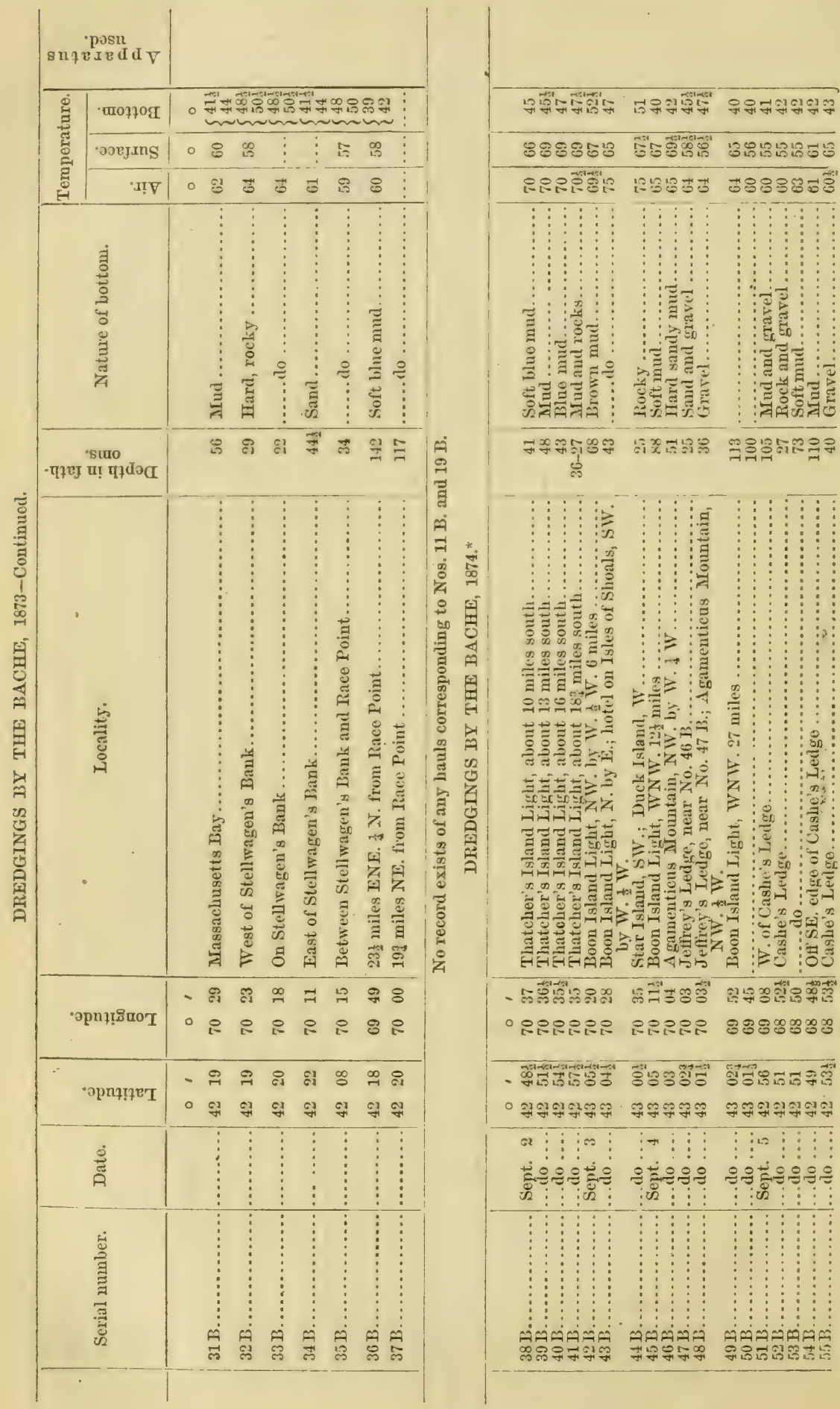




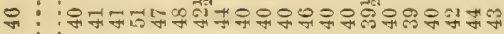

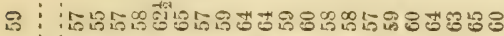

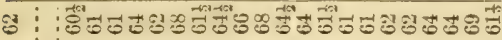
ค่。ี 婂:

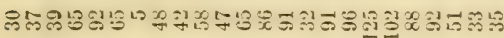

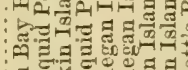

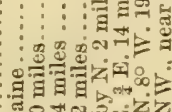

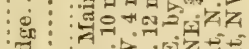

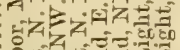

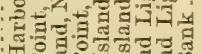
:

윙ㅇํㅇ: : 泣: : : :

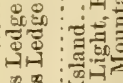
in

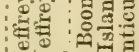
$\operatorname{lom}_{-\infty}^{\infty} 0$ कै० a

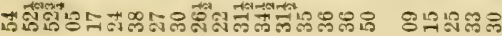

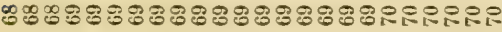

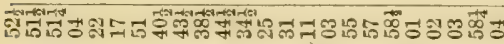

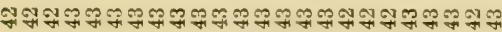

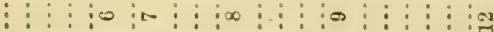

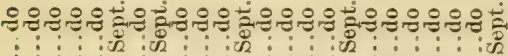

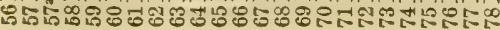

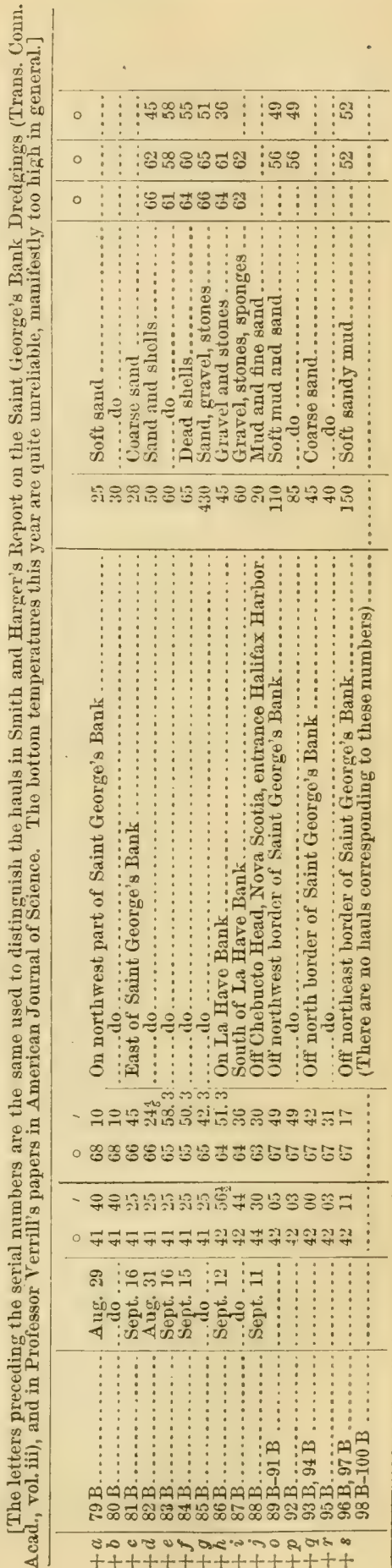




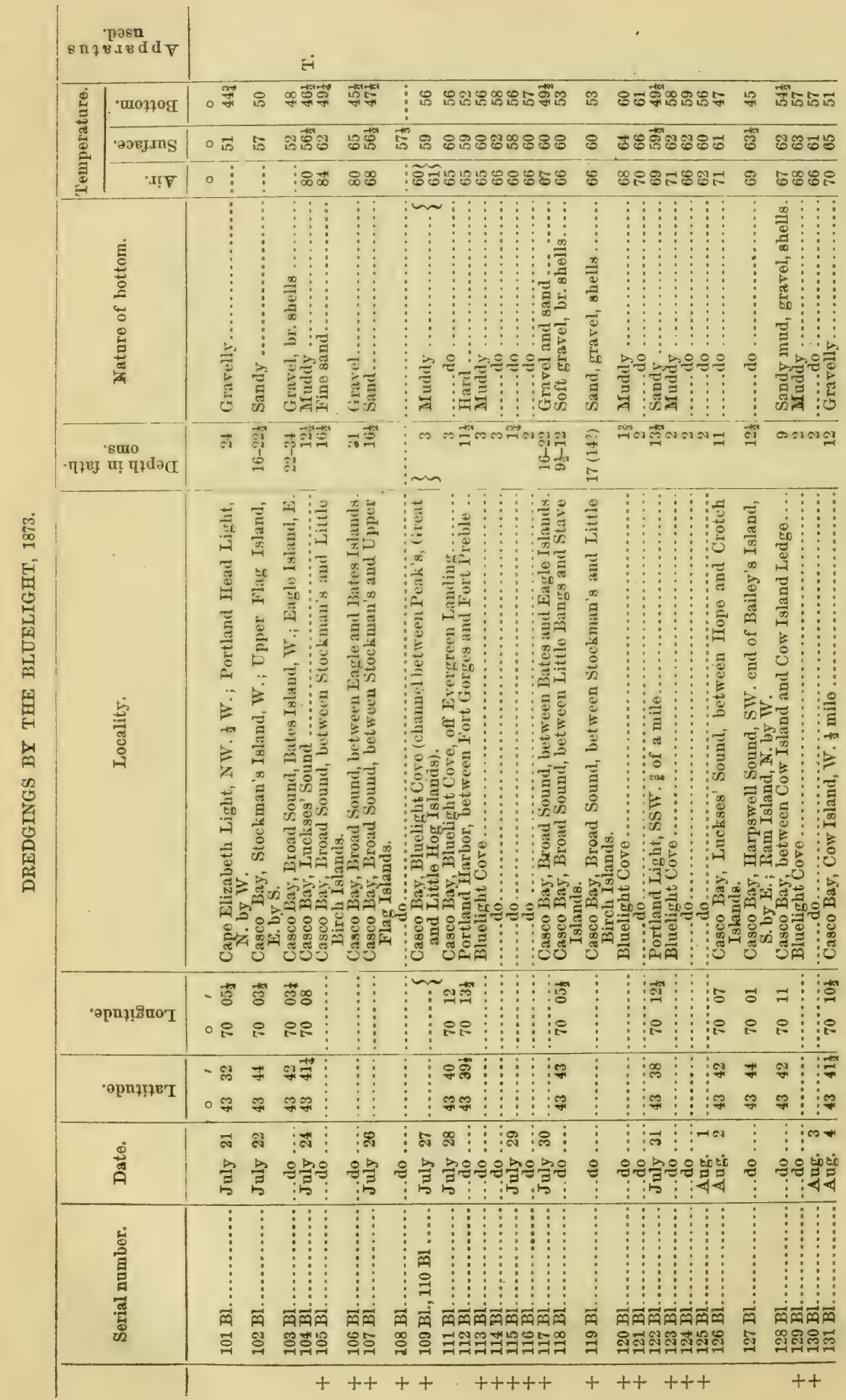




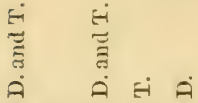

छं

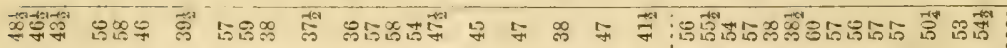

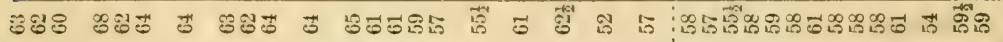

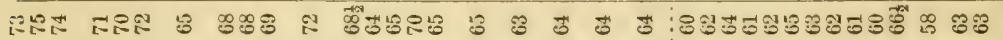

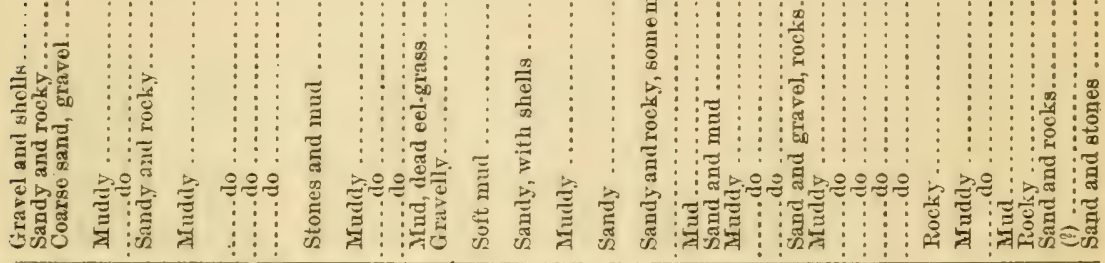

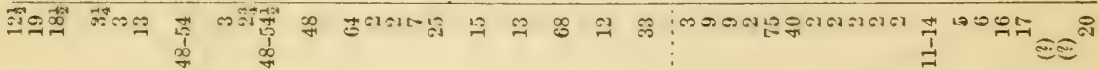
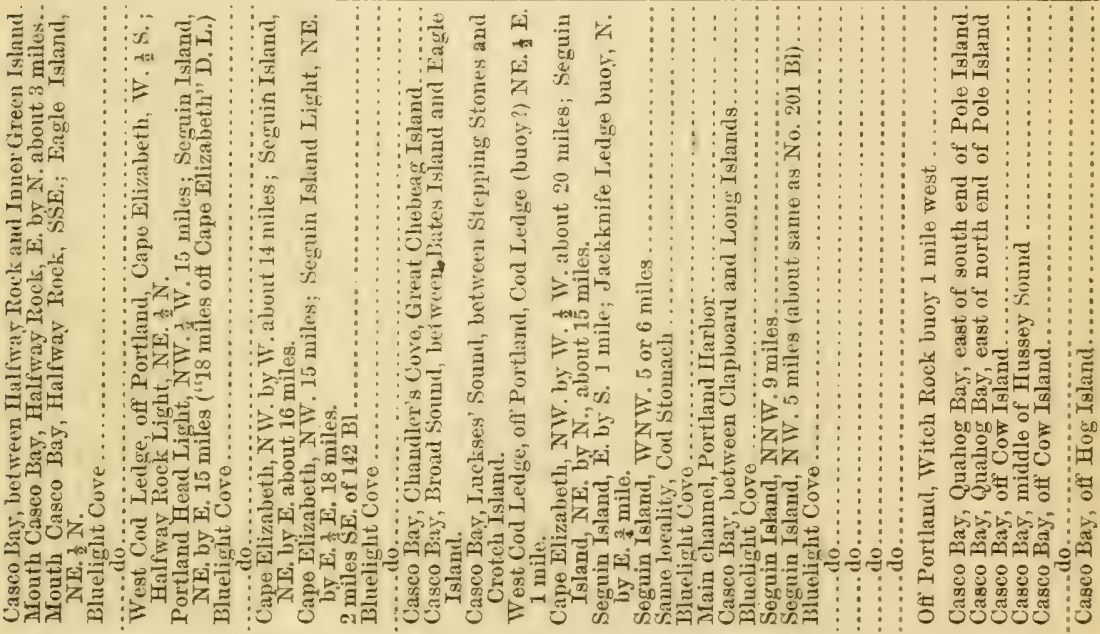

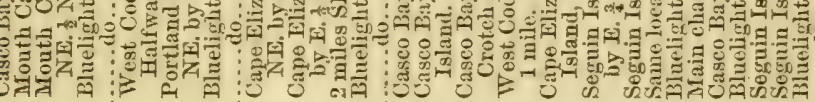

రు

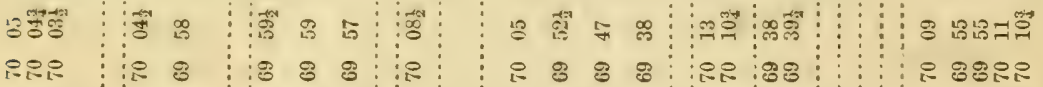

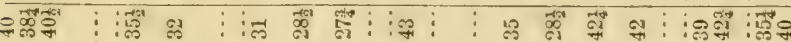

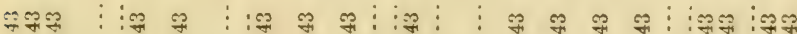

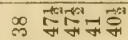

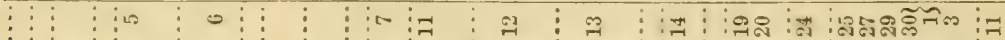

อง

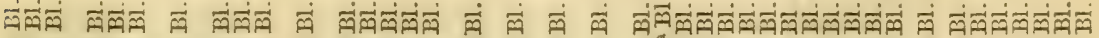

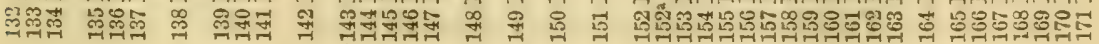




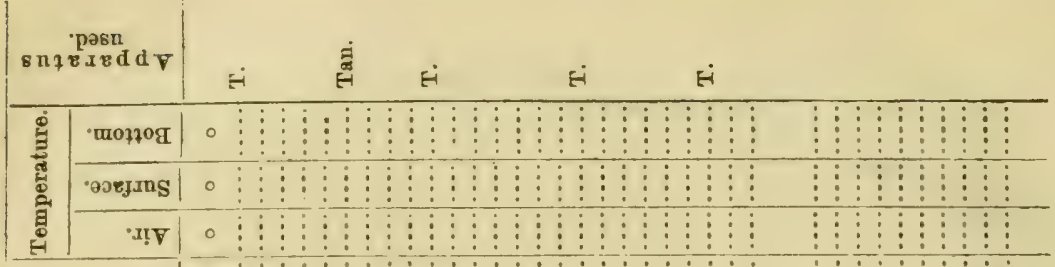

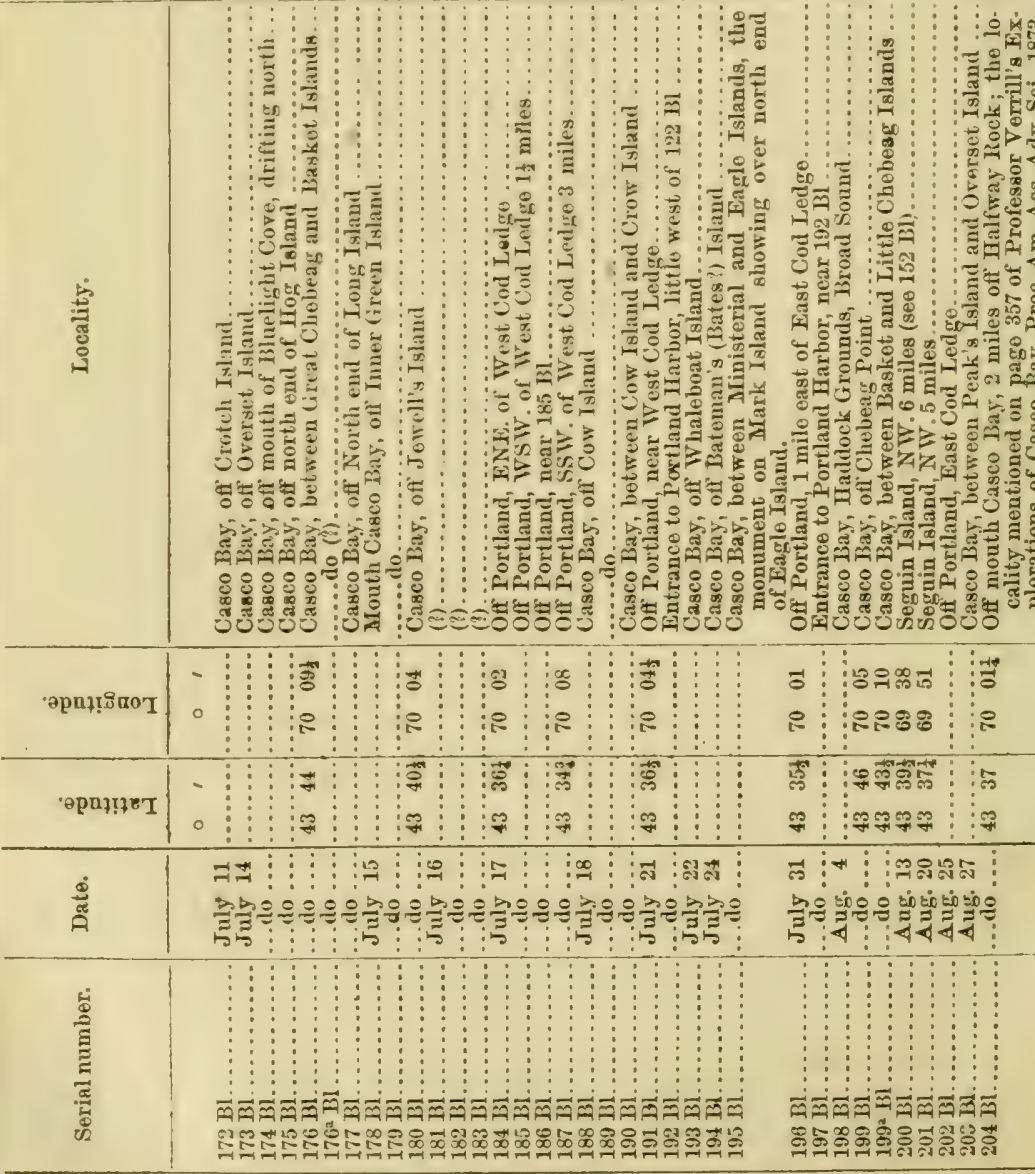




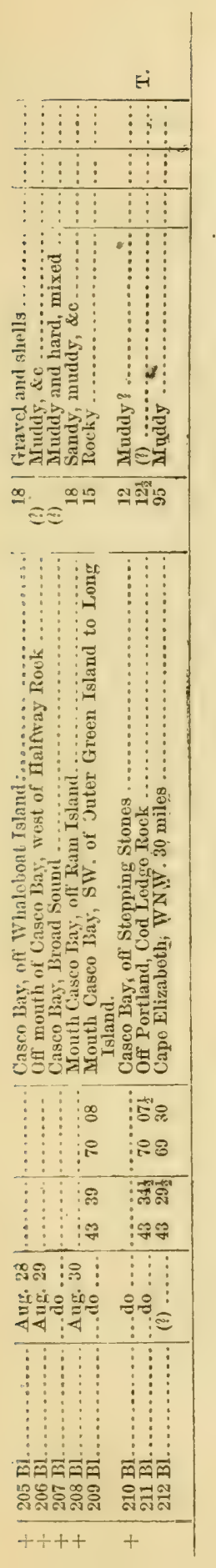


STATIONS FOR 1874 AND 1875, WITH HEADQUARTERS AT NOANK, CONN., AND WOOD'S HOLL, MASS.

In 1874, the headquarter's of the United States Fish Commission were established at Noank, Conn., and the area covered by its dredgings included Fisher's Island Sound; the eastern part of Long Island Sound; Block Island Sound; and Gardiner's and Peconic Bays; and also extended some distance to the east, sonth, and southwest of Block Island. In 1875, with headquarters at Wood's Holl, Mass., dredgings were carried on in Vineyard and Nantucket Sounds; Buzzard's Bay; over a portion of Nantucket Shoals; to the sonthward of Nantucket Island and Martha's Vineyard; and also on and about Southwest Shoal. The dredgings were all made by the United States steamer Bluelight, Commander L. A. Beardslee, and a separate series of numbers, to designate the stations, was employed for each jear. To facilitate the recording of all the dredging stations of the United States Fish Commission on charts, and to bring the sonthern ones into uniformity with those made to the north of Cape Cod in more recent years, and already recorded both on charts and in reports prepared for publication in a single series of numbers ranging from 1 to 375,400 has been added to the 1874 dredgings and 600 to those of 1875 . In this way all the dredging stations from 1874 to 1579 , inclusive, are included in a single series.

The temperature observations recorded in the two following tables were mostly taken with much care. Former txperiences had proved that the Miller-Casella thermometers were slow in acting, requiring from three to ten minutes (according to the rlepth of water) to obtain a correct reading, and they were, therefore, always left down a suitable length of time. The bottom and surface temper'atures, in nearly all eases, were taken with Miller-Casella self-registering thermometers; occasionally a United States naral thermometer was employed for surface temperatures, and the same instrument was generally employed for the air.

STATIONS FOR 1874.

\begin{tabular}{|c|c|c|c|c|c|c|c|c|}
\hline \multirow{2}{*}{ 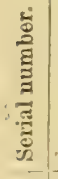 } & \multirow[b]{2}{*}{ Date. } & \multirow[b]{2}{*}{ Locality. } & \multirow{2}{*}{ 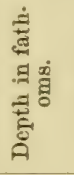 } & \multirow[b]{2}{*}{ Nature of bottom. } & \multicolumn{3}{|c|}{ Temperatures. } & \\
\hline & & & & & $\frac{\pi}{4}$ & 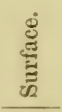 & 苛 & \\
\hline 401 & $\begin{array}{l}1874 . \\
\text { July } 13\end{array}$ & $\begin{array}{l}\text { Fishers Island Sound, West } \\
\text { Clump, bearing S. }\end{array}$ & $7 \frac{1}{2}$ & Mrud ....... & $\circ$ & - & 。 & \\
\hline & $\begin{array}{l}13 \\
13 \\
13\end{array}$ & $\begin{array}{l}\text { Fisher's Island Sound } \\
\text {. . . }\end{array}$ & $\begin{aligned} 9 \frac{2}{2} \\
11 \frac{1}{2}\end{aligned}$ & $\begin{array}{l}\text { Sand.. } \\
\ldots \text { do. }\end{array}$ & & & & \\
\hline $\begin{array}{l}405 \\
406\end{array}$ & July it & $\begin{array}{l}\text { Fislier's Isliund Sound, off Lati- } \\
\text { mer's libef. } \\
\text { (No record.) } \\
\text { Eisher's Istand Sound, N. of } \\
\text { Young's Rock. }\end{array}$ & 11 & Rocky.. & & & & \\
\hline 407 & 14 & Fisher's Island Sound, NW. of & 9 & Sand, stones & & & & \\
\hline 408 & 14 & $\begin{array}{l}\text { Fisher's Island Sound, N. by E. } \\
\text { of W icopessit. }\end{array}$ & $11 \frac{1}{2}$ & Clay ..... & & & & D. \\
\hline 409 & 14 & $\begin{array}{l}\text { Fisher's Island Sound, Lord's } \\
\text { Channel. }\end{array}$ & $11 \frac{1}{3}$ & Rocky .... & & & & Tan \\
\hline 410 & 14 & $\begin{array}{l}\text { Fishor's Island Sound, off Nap- } \\
\text { atree Point }\end{array}$ & $2 \frac{1}{2}$ & Sand..... & & & & $\mathbf{T}$. \\
\hline 411 & 16 & $\begin{array}{l}\text { atree Point. } \\
\text { Watch Hill Light-House, R. I., } \\
\text { NN W., distaut about S' mile. }\end{array}$ & 11 & ....do ... & & & & \\
\hline
\end{tabular}


STATIONS FOR 1874-Continued.

\begin{tabular}{|c|c|c|c|c|c|c|c|c|}
\hline : & & & & & Tem & peratr & ires. & \\
\hline$\underset{\Xi}{\tilde{\Xi}}$ & Date. & Locality. & हैं & Nature of bottom. & यี่ & 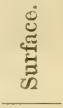 & $\underset{\tilde{n}}{\stackrel{\Xi}{\tilde{n}}}$ & 宷 \\
\hline 412 & $\begin{array}{l}1874 . \\
\text { July } 16\end{array}$ & $\begin{array}{l}\text { Watch Hill Light NNE., distant } \\
\text { nearly } \frac{1}{2} \text { mile. }\end{array}$ & 5 & Rocky ... & $\circ$ & 0 & ० & Tan. \\
\hline $\begin{array}{l}413 \\
414\end{array}$ & $\begin{array}{l}16 \\
16\end{array}$ & $\begin{array}{l}\text { Fisher's Island Sound, off Gro. } \\
\text { ton Long Point. }\end{array}$ & $\begin{array}{l}5 \\
7\end{array}$ & Gravel.... & & & & $\begin{array}{l}\text { Tan. } \\
\text { D. }\end{array}$ \\
\hline 415 & 16 & $\begin{array}{l}\text { Fisher's Island Sound, Groton } \\
\text { Long Point NW. by N. } \frac{1}{2} \text { mile. }\end{array}$ & 7 & ....do & & & & D. \\
\hline 116 & 17 & $\begin{array}{l}\text { Fisher's Island Sound, mile W. } \\
\text { by N. of N. Hammock Light- } \\
\text { House. }\end{array}$ & 6 & Sand, mud. & & & & D. \\
\hline 417 & 17 & $\begin{array}{l}\text { Long Island Sound, New Lon- } \\
\text { don Light } \mathrm{N} \text {. by } \mathrm{W} \text {., distant } \\
\text { about } 2 \frac{1}{4} \text { miles. }\end{array}$ & 8 & Sand... & & & & D. \\
\hline 418 & 17 & $\begin{array}{l}\text { Long Island Sound, New Lon- } \\
\text { don Light N., distant } 17 \text { miles. }\end{array}$ & 9 & Sand, muat.. & & & & $\mathbf{T}$. \\
\hline 419 & 17 & $\begin{array}{l}\text { Long Island Sound, Little Gull } \\
\text { Island Light bearing S. by. E. } \\
2 \text { miles. }\end{array}$ & 40 & Gravel. & & & & D. \\
\hline 420 & 20 & $\begin{array}{l}\text { Fisher's Island Sound, } \frac{1}{4} \text { mile N. } \\
\text { of West Clump. }\end{array}$ & $11_{\frac{1}{2}}^{1}$ & ...do ....... & ...... & & & D. \\
\hline 421 & 20 & $\begin{array}{l}\text { Fisher's Island Sound, N. Ham- } \\
\text { mock Light W. by S. } 1 \text { mile. }\end{array}$ & $12 \frac{1}{2}$ & Sand, gravel .. & & & & D. \\
\hline 422 & 20 & $\begin{array}{l}\text { Fisher's Island Sound, N. Ham. } \\
\text { mock Light S. by W. } \mathrm{W} \text {. W. } \frac{1}{4} \\
\text { mile. }\end{array}$ & 13 & .... do . & & $\cdots$ & & D. \\
\hline 423 & 20 & $\begin{array}{l}\text { Fisher's Island Sound, N. Ham. } \\
\text { mock Light E. } \frac{1}{3} \text { mile. }\end{array}$ & 17 & Gravel...... & & & & D. \\
\hline 424 & 20 & $\begin{array}{l}\text { Fisher's Island Sound, N. Ham- } \\
\text { mock Light E. by N. } 1 \text { mile. }\end{array}$ & $7 \frac{1}{2}$ & Sand, mud ... & & & & D. \\
\hline 425 & 20 & 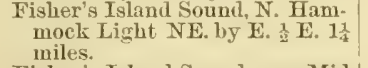 & $10_{4}^{3}$ & Mud... & & $\cdots$ & & D. \\
\hline 426 & 20 & $\begin{array}{l}\text { Fisher's Island Sound, near Mid- } \\
\text { dle Clump. }\end{array}$ & 8 & Sand.......... & & & & T. \\
\hline 127 & 22 & $\begin{array}{l}\text { Fisher's Island Sound, } \frac{1}{4} \text { mile } \\
\text { NW. of Middle Clmmp. }\end{array}$ & $11 \frac{1}{2}-9 \frac{x}{2}$ & Sand, shells ...... & 65 & 64 & 62.5 & D. \\
\hline $4 \div 8$ & 22 & $\begin{array}{l}\text { Fisher's Island Sound, } 1 \text { mile } \\
\text { NNTW. of Middle Clump. }\end{array}$ & 11 & .....do ........ & 65 & 64 & 62.5 & D. \\
\hline 429 & 22 & $\begin{array}{l}\text { Fisher's Island Sound, } 1 \text { milo } \\
\text { NNE. of W. Clump. }\end{array}$ & 8 & ... do .. & 65 & 64 & 63 & D. \\
\hline 430 & 23 & $\begin{array}{l}\text { Fisher's Island Sound, Eelgrass } \\
\text { Light-Ship E. by } \mathrm{W}^{\mathrm{V}} \text {, distant } \frac{1}{2} \\
\text { mile. }\end{array}$ & 7 & Sand, gravel ...... & 66 & 64 & 62.5 & D. \\
\hline 431 & 23 & $\begin{array}{l}\text { Fisher's Island Sound, between } \\
\text { Latimer's Ieef and Young's } \\
\text { Rock. }\end{array}$ & $10 \frac{1}{2}$ & Sand, gravel, shells. & 65.5 & $\begin{array}{l}62.5 \\
.\end{array}$ & 61.5 & D. \\
\hline 432 & 23 & $\begin{array}{l}\text { Fisher's Island Sound, eastward } \\
\text { of Latimer's Reef. }\end{array}$ & 11 & $\begin{array}{l}\text { Coarse sand, shells, } \\
\text { rocks. }\end{array}$ & 65 & 62.5 & 61 & D. \\
\hline 433 & 24 & $\begin{array}{l}\text { Tisher's Island Sound, Groton } \\
\text { Long Point NE. by N., distant } \\
\frac{1}{8} \text { mile. }\end{array}$ & 8 & Sand, shells ....... & 72 & 66 & 63 & D. \\
\hline 131 & 24 & $\begin{array}{l}\text { Fisher's Island Sound, between } \\
\text { Sea-Flower Reef and Groton } \\
\text { Long Point. }\end{array}$ & 7 & . . . do ................ & 71 & 65.5 & 62.5 & D. \\
\hline 435 & 24 & $\begin{array}{l}\text { Long Island Sound, Raco Point } \\
\text { bearing E, distant } 2 \frac{1}{3} \text { miles. }\end{array}$ & 50 & $\begin{array}{l}\text { Rocky, with mus. } \\
\text { sels. }\end{array}$ & 72 & 68 & 59 & D. \\
\hline 436 & 24 & $\begin{array}{l}\text { Long Island Sound, about } \frac{1}{4} \text { milo } \\
\text { SW of } 435 \text {. }\end{array}$ & 50 & Rocks, gravel..... & 68 & 68 & 58 & D. \\
\hline 437 & 24 & $\begin{array}{l}\text { Block Island Sound, off Culloden } \\
\text { Point, Long Islaud. }\end{array}$ & $12 \frac{1}{2}$ & Sand, mud ........ & 74 & 60 & 61 & $\mathrm{~T}$. \\
\hline 438 & 24 & $\begin{array}{l}\text { Block Island Sound, N TV of Cul-1 } \\
\text { loden Point, Long Island. }\end{array}$ & 12 & Sand... & & & & D. \\
\hline 439 & 27 & $\begin{array}{l}\text { Fisher's Island Sound, eastern } \\
\text { part of Sweeper Sound. }\end{array}$ & 4 & Sand, shells. & 70.5 & 66.5 & 65 & D. \\
\hline 440 & 27 & $\begin{array}{l}\text { Fisher's Island Sound, house on } \\
\text { Ram Island bearing NE. } 1 \text { E. }\end{array}$ & 4 & Sand... & 68.5 & 66.5 & 65.5 & D. \\
\hline 441 & 27 & $\begin{array}{l}\text { Fisher's Island Soumd, SW. of } \\
\text { Ram Island } \frac{1}{4} \text { mile. }\end{array}$ & $3 \frac{1}{2}$ & ....do. & 68.5 & 66.5 & 65 & D. \\
\hline 443 & 27 & $\begin{array}{l}\text { Fisher's Island Sound, off Middle } \\
\text { Clump. }\end{array}$ & 14 & Stones, grarel..... & 68.5 & 66.5 & 61 & D. \\
\hline $\begin{array}{l}443 \\
444\end{array}$ & $\begin{array}{l}27 \\
29\end{array}$ & $\begin{array}{l}\text { Fisher's Island Sound, NW. of } \\
\text { Eel grass Light-Ship, distant } \\
\text { about } 8 \text { mile }\end{array}$ & $\frac{10 \frac{1}{2}}{7}$ & Sand, gravel, shells & $\begin{array}{l}67 \\
\cdots \cdots\end{array}$ & 66 & 64.5 & D. \\
\hline
\end{tabular}


STATIONS FOR 1874-Continued.

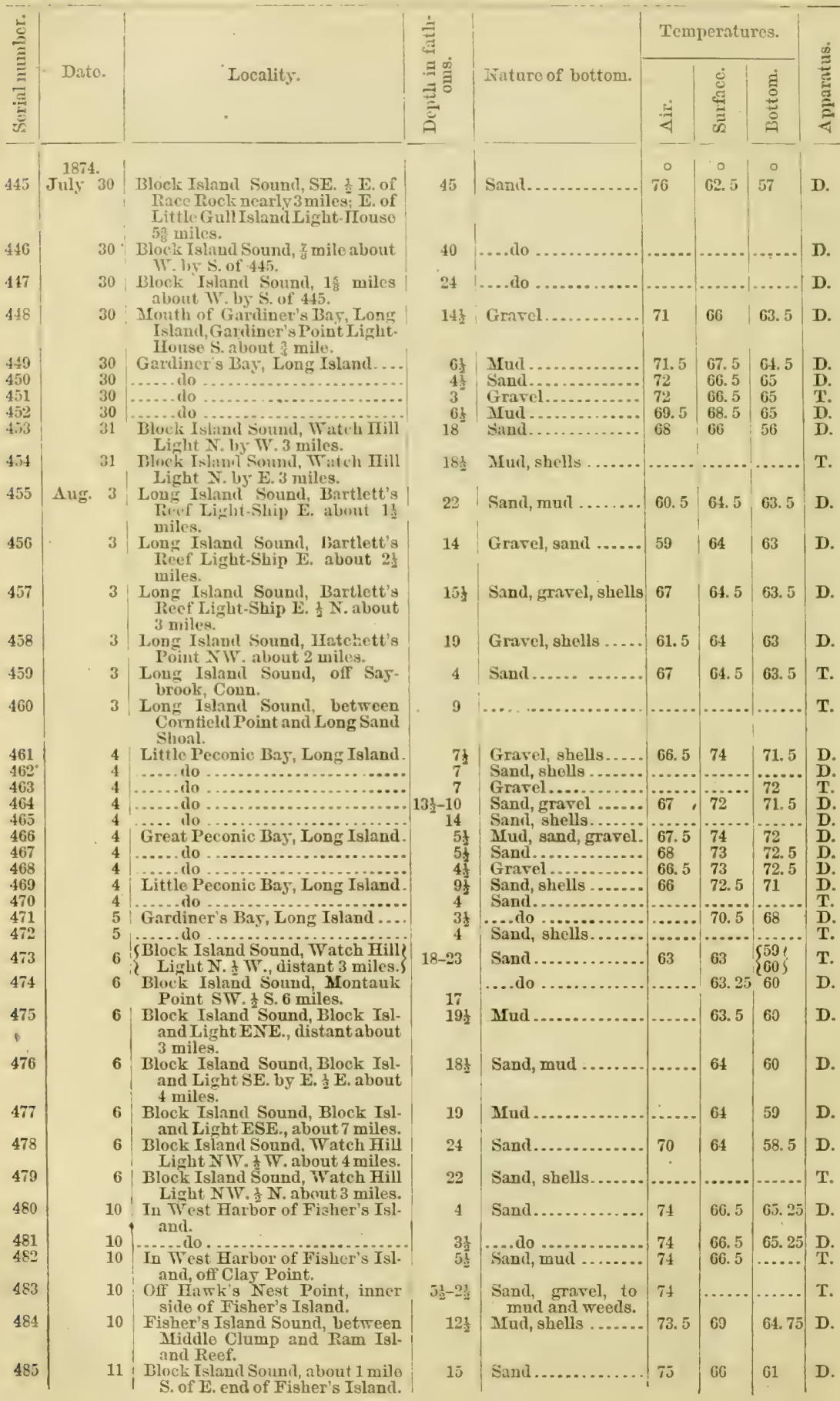


STATIONS FOR 1874-Continued

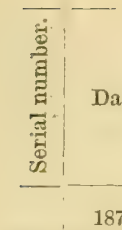

1874.

486 Ang. 11 Block Islund Sound, about mile S. of E. end of Fisher's Island. Block Island Sound, about $\frac{1}{2}$ mile

\begin{tabular}{ll|l} 
& ott' centre of Fisher's Island.
\end{tabular} Prospect, Fisher's Island, about $\frac{1}{4}$ mile from land.
Block Island Sound, about $\frac{1}{2}$ mile westward of 488 .

11 Block Island Sound, about $\frac{3}{8}$ mile SE. of Race Point.

11 Block Island Sound, about $1 \frac{1}{4}$ miles S. of Mount Prospect.

12 Noank Harbor.................... Sea-Flower and Horse-Shoe Reefs.

12 Fisher's Island Flower Reef Beacon.

12 Fisher's Island Sound, TV. of SeaFlower Reef Beacon about 1 mile.

13 Block Island Sound, Montauk Point Light SSE. about 6 miles.

13 Block Island Sound. Montauk Point Light SSE. about $6:$ miles.

13 Block Island Sound, Montauk Point Light SSE. about $7 \frac{1}{3}$ miles.

13 Block Island Sound, Montauk Point Light $\mathrm{S}$. by E. 48 miles.

13 Block Island Sound, Montauk Point Liglit S. by iV sabout 3 miles.

13 Bloek Island Sound, Montauk Point Light SSIT. about 23 miles.

13 OffMontauk Point, Light-House WSW. about 2 miles.

13 Off Montauk P'oint, Light-House IV. about '2 miles.

14 Fisher's Island Sound, between Eelgrass Liglit-Ship and White Rock

14 Fisher's Island Sound, about 1 mile E. by $\mathbf{N}$. from Eelgrass Light-Ship.

14 Fisher's Island Sound, Stonington Iight NE. $\frac{1}{2}$ E about 1 mile.

14 Fisher's Islaud Sound, Eelgrass Light-Ship IVNW. mile.

17 Fislier's Island Sound, Eelgrass Light-Ship NT. by W. about $\frac{3}{4}$ mile.

17 Fisher's Island Sound, Eelgrass Light.Ship IV N W. 1 mile.

17 Fishex's Island Sound, Eelgrass Light-Ship W. by N. about $1 \frac{1}{4}$ miles.

17 Fisher's Island Sound, Stonington Light ENE, about $1 \frac{1}{4}$ miles.

17 Fisher's Island Sound, Eelgrass Light-Ship WV. $\frac{1}{3} \mathrm{~N}$. about 1 mile.

17 Fishtr"s Island Sound, Eelgrass Limht-Ship $\mathrm{E}$. about 1 mile.

18 Off Block Island, Montauk Point TV. about 9 miles.

18 Off Block Island, Montauk Point NTV. by W. $\frac{1}{2}$ W. about 11 miles.

18 off lo Off Block Islani, old Harbor
Point, Block Island N. 5 miles.
Fisher's Island Sound, between

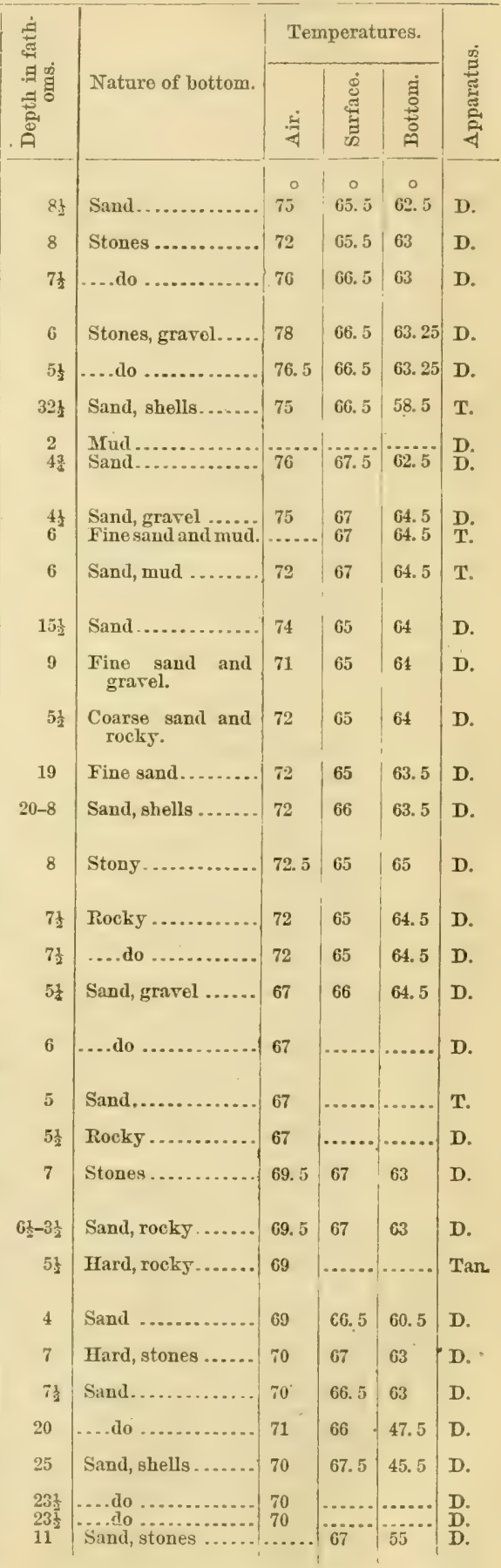


STATIONS FOR 1874-Continued.

\begin{tabular}{|c|c|c|c|c|c|c|c|c|}
\hline$\ddot{\leftrightarrows}$ & & & & & Tem & peratu & res. & \\
\hline$\underset{\pi}{3}$ & Dato. & Locality: & 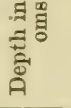 & Nature of bottom. & $\stackrel{ت}{4}$ & 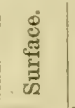 & $\frac{d}{0}$ & 蒫 \\
\hline 520 & $\begin{array}{l}1874 . \\
\text { Aug. } 18\end{array}$ & $\begin{array}{l}\text { Off Block Island; Old Harbor } \\
\text { Point, Block Island, N. } 5 \text { miles. }\end{array}$ & 11 & Sand, stones. & $70^{\circ}$ & o & $\circ$ & D. \\
\hline $\begin{array}{l}521 \\
522\end{array}$ & 18 & off New Shoreham, Block Island & 14 & Gravel, stones & 70 & 66 & 57.5 & D. \\
\hline $\begin{array}{l}522 \\
523\end{array}$ & $\begin{array}{l}18 \\
19\end{array}$ & $\begin{array}{l}\text { Off Block Island, New Shoreham } \\
\text { NIV. by N. about } 6 \text { miles. }\end{array}$ & $\begin{array}{l}18 \\
14\end{array}$ & 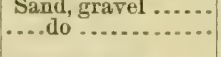 & $\begin{array}{l}70 \\
73\end{array}$ & $\begin{array}{l}66.5 \\
66.5\end{array}$ & $\begin{array}{l}52.5 \\
54\end{array}$ & $\begin{array}{l}\text { D. } \\
\text { D. }\end{array}$ \\
\hline 521 & 19 & $\begin{array}{l}\text { Otir Block Island, New Shoreham } \\
\text { NNW. }\end{array}$ & $14 \frac{1}{2}$ & Coarse sand & 73 & 66 & 50.5 & D. \\
\hline 525 & 19 & Off Block Island, SE. side ....... & $14 \frac{1}{2}$ & Gravel....... & 69.5 & 66.5 & 53 & D. \\
\hline 526 & 19 & $\begin{array}{l}\text { SE. from Point Judith, Rhodo } \\
\text { Island, aboút } 4 \text { miles. }\end{array}$ & $13 \frac{1}{2}$ & Saud, gravel. & 75 & 67.5 & 54.5 & D. \\
\hline 527. & 19 & $\begin{array}{l}\text { S. from Point Judith, Rhode Isl- } \\
\text { and, about } 2 \frac{1}{2} \text { miles. }\end{array}$ & 9 & Stones. & & 69.5 & 61 & D. \\
\hline 528 & 19 & $\begin{array}{l}\text { W. from Point Juditb, Rhode } \\
\text { Island, about } 3 \text { miles. }\end{array}$ & 4 & Rocks, sand... & 76 & 67.5 & 63 & D. \\
\hline 529 & 19 & $\begin{array}{l}\text { Oti Yarragansett Beach, Rhode } \\
\text { Issland. }\end{array}$ & $8 \frac{1}{2}$ & Sand, gravel. & & & & T. \\
\hline 530 & 19 & ..... do do....................... & $10 \frac{1}{2}$ & Stones, gravel..... & & & & T. \\
\hline 531 & 21 & $\begin{array}{l}\text { Block Island Sound, Watch Hill } \\
\text { Light } N . \frac{1}{2} \mathrm{E}_{0} \text {, distant } 3 \text { miles. }\end{array}$ & 21 & Sami & 80 & 67.25 & 56.5 & D. \\
\hline 532 & 21 & $\begin{array}{l}\text { Block Island Sound, S IV. } 3 \text { S. of } \\
\text { No. 531, distant t mile. }\end{array}$ & 20 & .... do .. & 80 & 67.25 & & T. \\
\hline 533 & 21 & $\begin{array}{l}\text { Block Island Sound, WVSW. of } \\
\text { No. } 531 \text {, distant } \frac{5}{8} \text { mile. }\end{array}$ & $17 \frac{1}{2}$ & ....do . . & 79.5 & 67.25 & & T. \\
\hline 534 & 21 & $\begin{array}{l}\text { Block Island Sound about S. } ~ E \text {. } \\
\text { of east point of Fisher's Island } \\
7 \text { milo. }\end{array}$ & 9 & Gravel........ & 78 & 66.5 & 63.5 & D. \\
\hline 535 & 21 & $\begin{array}{l}\text { Block Island Sound, east end of } \\
\text { Fisher's Island N. by E. about } \\
2 \text { miles. }\end{array}$ & $19 \frac{1}{2}$ & Sand... & 78 & 67 & 57.5 & D. \\
\hline 536 & 24 & $\begin{array}{l}\text { Fort Pond Bay, east end of Long } \\
\text { Island. }\end{array}$ & $7 \frac{1}{2}$ & Mud........ & 76 & 73.5 & 65.5 & \\
\hline 537 & 24 & $\begin{array}{l}\text { Otf Fort Pond Bay, east end of } \\
\text { Long Ishand. }\end{array}$ & $6 \frac{1}{2}$ & Sand, gravel & & & $\ldots \ldots$ & T. \\
\hline 538 & 24 & $\begin{array}{l}\text { Napeague Bay, off Culloden } \\
\text { Point, Long Tsland. }\end{array}$ & $8 \frac{1}{2}$ & Sayd.. & & 67.5 & 65.5 & D. \\
\hline 539 & 24 & $\begin{array}{l}\text { Napeague Bay, east end of Long } \\
\text { Island. }\end{array}$ & $5-8$ & Mo 0 & & 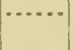 & .... & $\mathrm{T}$. \\
\hline $\begin{array}{l}540 \\
541\end{array}$ & $\begin{array}{l}24 \\
24\end{array}$ & Block Islani Souni, Irace Point & $\begin{array}{r}6-7 \\
42\end{array}$ & Stony $\ldots$............ & 70.5 & 66 & & $\begin{array}{l}\text { D. } \\
\text { D. }\end{array}$ \\
\hline 542 & 25 & $\begin{array}{l}\text { N. about } 1 \frac{1}{8} \text { mile. } \\
\text { Off Hay Harbor, west end of } \\
\text { Fisher's Island. }\end{array}$ & $4 \frac{1}{2}$ & Sand... & 70 & 65.5 & 64.5 & D. \\
\hline 543 & 25 & $\begin{array}{l}\text { Off west end of Fisher's Island, } \\
\text { liace Point about S., distant } \frac{1}{2} \\
\text { mile. }\end{array}$ & $7 \frac{1}{2}$ & Mud, sand... & 70 & 65.5 & 64.5 & D. \\
\hline 544 & 25 & $\begin{array}{l}\text { Off' west end of Fisher's Island, } \\
\text { Race Point SSE. } 1 \text { mile. }\end{array}$ & $8 \frac{1}{2}$ & Fine sand.... & & & & $\mathbf{T}$ \\
\hline 545 & 25 & $\begin{array}{l}\text { Off west end of Fisher's Island, } \\
\text { Race Point about S. mile. }\end{array}$ & $5 \frac{1}{2}$ & Rocks & & & & D. \\
\hline 546 & 25 & $\begin{array}{l}\text { Fisher's Island Sound, between } \\
\text { Cast Clump and Ram Island } \\
\text { buoy, }\end{array}$ & $7 \frac{1}{2}$ & Hard . & 74.5 & 65.5 & 65 & D. \\
\hline 548 & 25 & $\begin{array}{l}\text { Fisher's Island Sound, ESE. } \\
\text { from house on Ram Island. }\end{array}$ & $7 \frac{1}{2}$ & & & & & $\mathrm{D}$. \\
\hline 549 & 27 & $\begin{array}{l}\text { Off Niantic 13ay, Connecticut, } \\
\text { WV of Two-Tree Island. }\end{array}$ & 5 & Sand... & 70.5 & 65 & 64 & D. \\
\hline 550 & 27 & $\begin{array}{l}\text { Ofr Niantic Bay, Connecticut, } \\
\text { between Black Point and Two- } \\
\text { Tree Island. }\end{array}$ & $5 \frac{1}{2}$ & ....do ... & $\ldots$ & $\ldots \ldots$ & & T. \\
\hline $\begin{array}{l}551 \\
552\end{array}$ & $\begin{array}{l}27 \\
27\end{array}$ & Long Island Sound, off Saybrook & $5 \frac{1}{2}$ & ... do .. & & & & $\mathrm{D}$. \\
\hline 553 & & $\begin{array}{l}\text { Long Island Sound, Saybrook } \\
\text { Light NE. } 2 \text { miles. }\end{array}$ & $7 \frac{1}{2}$ & .... do ... & & & & $\mathrm{T}$. \\
\hline 554 & 27 & $\begin{array}{l}\text { Long Island Sound, Plum Island } \\
\text { Light SE. by E. } 3 \text { miles. }\end{array}$ & 22 & Gravel.... & 73.5 & 66 & 65 & D. \\
\hline $\begin{array}{l}555 \\
556\end{array}$ & .27 & Off CorLedge ESE, from Block & 26 & \#...do... & 73.5 & 66 & 65 & D. \\
\hline & & $\begin{array}{l}\text { Island about } 20 \text { miles. } \\
\text { (The shallowest part of Cox } \\
\text { Ledge lies in about } 11^{1} / \mathrm{N} \text {. } \\
\text { Lat. and } 71^{\circ} 02^{\prime} \mathrm{W} \text {. Long.) }\end{array}$ & 20 & Gom - & & ..... & & D. \\
\hline $\begin{array}{l}557 \\
558\end{array}$ & $\begin{array}{l}30 \\
30\end{array}$ & $\begin{array}{l}\text { Off Cox Ledge } \ldots \ldots \ldots \\
\ldots\end{array}$ & $\begin{array}{l}21_{\leftarrow} \\
21\end{array}$ & $\begin{array}{l}\text { Sand, rocks ........ } \\
\text {.... do ............. }\end{array}$ & 67 & 62 & 51.5 & $\begin{array}{l}\text { D. } \\
\text { Tan }\end{array}$ \\
\hline 559 & 30 & ...... do ............ & 21 & & & & & \\
\hline
\end{tabular}


STATIONS FOR 1874-Coneluded.

\begin{tabular}{|c|c|c|c|c|c|c|c|c|c|}
\hline \multirow{2}{*}{ 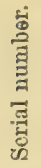 } & \multirow{2}{*}{\multicolumn{2}{|c|}{ Date. }} & \multirow[b]{2}{*}{ Loeality. } & \multirow{2}{*}{ 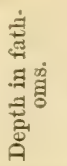 } & \multirow[b]{2}{*}{ Nature of bottom. } & \multicolumn{3}{|c|}{ Temperatures. } & \multirow{2}{*}{ 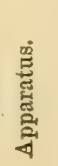 } \\
\hline & & & & & & $\ddot{4}$ & 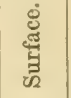 & 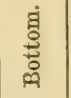 & \\
\hline 560 & $\begin{array}{r}187 \\
\text { Aug. }\end{array}$ & & & 21 & Rack & ○ & 0 & 0 & Tan. \\
\hline 561 & & 30 & $\begin{array}{l}\text { Old Harbor Point, Block Island. } \\
\text { About } 10 \text { miles SE. by E. from } \\
\text { Old Harbor Point, Block Island. }\end{array}$ & 34 & 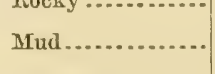 & 70 & 64 & 52 & D. \\
\hline 562 & & 30 & 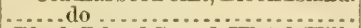 & 34 & $\ldots$ do $\ldots \ldots \ldots . . . . .$. & 70 & 64 & 52 & D. \\
\hline 563 & & 31 & $\begin{array}{l}\text { Block Tsland Sound, Watch Hill } \\
\text { Light } \mathrm{N} \text {. by W. abont } 3 \frac{1}{3} \text { miles. }\end{array}$ & 19 & Sand ................... & $\ldots \ldots$ & $\ldots \ldots$ & $\ldots \ldots$ & $\mathrm{T}$ \\
\hline 564 & & 31 & $\begin{array}{l}\text { Block Island Sound, Watch Hill } \\
\text { Light N. by W. } \mathrm{W} \text {. about } 3 \frac{1}{4} \\
\text { miles. }\end{array}$ & 18 & Sand.............. & $\ldots \ldots$ & $\ldots \ldots$ & $\ldots \ldots=$ & O. $\mathbf{T}$. \\
\hline 565 & & 31 & $\begin{array}{l}\text { Block Island Sound, Watch Hill } \\
\text { Light NNIV.about } 3 \frac{3}{8} \text { miles. }\end{array}$ & 17 & 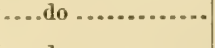 & $\ldots \ldots$ & $\ldots \ldots$ & $\ldots \ldots$ & O. T. \\
\hline 566 & & 31 & $\begin{array}{l}\text { Block Island Sound, W atch Hill } \\
\text { Light NN W. about } 3 \frac{2}{s} \text { miles. }\end{array}$ & 18 & $\ldots$. do $\ldots . . . . . . . . .$. & $\ldots \ldots$ & $=-\infty$ & $=-\infty \ldots$ & D. \\
\hline 567 & & 31 & . & 18 & ....do ................ & & & & D. \\
\hline 568 & Sept. & 2 & On Cox Ledge.................. & $19 \frac{1}{2}$ & Rocky ............. & $\ldots \ldots$ & $\ldots \ldots$ & 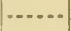 & D. \\
\hline 569 & & 2 & 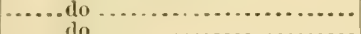 & 20 & Sand................... & $\cdots \cdots$ & (n...... & (....... & D. \\
\hline $\begin{array}{l}570 \\
571\end{array}$ & & $\begin{array}{l}2 \\
2\end{array}$ & . do & $\begin{array}{l}18 \frac{1}{3} \\
21\end{array}$ & 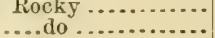 & …. & ( $\ldots \ldots$ & $\mid \cdots \cdots$ & D. \\
\hline 572 & & 2 & 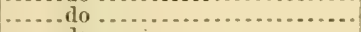 & 21 & .... do .............. & 72.5 & 62.5 & 50 & $\mathrm{D}$ \\
\hline 573 & & 2 & ...... do . . & $18 \frac{1}{2}$ & ... do .............. & $\ldots \ldots$ & $\ldots \ldots$ & $\ldots . .$. & D. \\
\hline 574 & & 2 & .... do . . . . & 19 & .... do ............. & ...... & ...... & ...... & $\mathrm{D}$. \\
\hline 575 & & 2 & 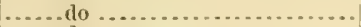 & 18 & ....do .............. & ..... & ....... & ...... & D. \\
\hline 576 & & 2 & 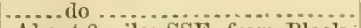 & 17 & .... do .............. & $\ldots \ldots$ & $\ldots \ldots$ & $\ldots \ldots$ & D. \\
\hline 577 & & 3 & $\begin{array}{l}\text { About } 8 \text { miles SSE. from Block } \\
\text { Island. }\end{array}$ & 19 & 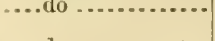 & $\ldots \ldots$ & $\ldots-\infty$ & $=\ldots-\infty$ & D. \\
\hline 578 & & 3 & $\begin{array}{l}\text { Crab Ledge, about } 7 \text { miles SE. of } \\
\text { Block Island. }\end{array}$ & $10 \frac{1}{2}$ & $\ldots$.... do $\ldots . . . . . . . . . .$. & ....... & $\ldots . .$. & $\ldots \ldots$ & D. \\
\hline 579 & & 3 & $\begin{array}{l}\text { Crab Ledge, about } 8 \text { miles SE. of } \\
\text { Block Island. }\end{array}$ & $10 \frac{1}{4}$ & ... do ............... & $\ldots \ldots$ & & & D. \\
\hline 580 & . & 3 & $\begin{array}{l}\text { A bout } 7 \text { miles off New Shoro- } \\
\text { ham, Btock Island. }\end{array}$ & $8-10$ & . . do $\ldots . . . . . . . . . .$. & $\ldots \ldots$ & $\ldots \ldots$ & $\ldots \ldots$ & D. \\
\hline
\end{tabular}

(There are no numbers 581-600.)

STATIONS FOR 1875.

\begin{tabular}{|c|c|c|}
\hline 601 & $\begin{array}{l}1875 \\
\text { July } 12\end{array}$ & $\begin{array}{l}\text { Vineyard Sound, Tarpaulin } \\
\text { Cove Liglit W. by S., Job's } \\
\text { Neck NE. by E. } \frac{1}{2} \text { E. }\end{array}$ \\
\hline 602 & 12 & $\begin{array}{l}\text { Vineyard Sound, Tarpaulin } \\
\text { Cove Light WSW. } 1 \text { mile. }\end{array}$ \\
\hline 603 & 12 & $\begin{array}{l}\text { Vineyard Sound, Tarpaulin } \\
\text { Cove Light NW. by W, } \frac{3}{6} \text { mile. }\end{array}$ \\
\hline 604 & 12 & $\begin{array}{l}\text { Vineyard Sound, Menemsha } \\
\text { Bight. }\end{array}$ \\
\hline 605 & 12 & 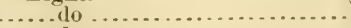 \\
\hline 606 & 12 & 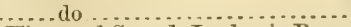 \\
\hline 607 & 14 & Vineyard Sound, Lackey's Bay. \\
\hline 608 & 14 & $\begin{array}{l}\text { Between Martha's Vineyardand } \\
\text { No Man's Land, Gay Head } \\
\text { Light N. } \frac{1}{2} \text { E. } 2 \frac{1}{2} \text { miles. }\end{array}$ \\
\hline 609 & 14 & $\begin{array}{l}\text { North of No Man's Land, Lone } \\
\text { Rock S. } \frac{1}{8} \text { mile. }\end{array}$ \\
\hline 610 & 14 & Nortl of No Man's Land ........ \\
\hline 611 & 14 & 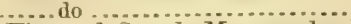 \\
\hline 012 & 14 & $\begin{array}{l}\text { Vineyard Sound, Menemsha } \\
\text { Biglit. }\end{array}$ \\
\hline 613 & 14 & $\begin{array}{l}\text { Vineyard Sound, Tarpaulin } \\
\text { Cove. }\end{array}$ \\
\hline 614 & 15 & $\begin{array}{l}\text { Vineyard Sound, S. and E. of } \\
\text { Davis Neck Shoal. }\end{array}$ \\
\hline 615 & 15 & $\begin{array}{l}\text { Vineyard Sound, S. of Monant } \\
\text { Point. }\end{array}$ \\
\hline 616 & 15 & $\begin{array}{l}\text { Vineyard Sound, off Davis Neck } \\
\text { Shoal. }\end{array}$ \\
\hline 617 & 15 & $\begin{array}{l}\text { Vineyard Sound, off East Chop } \\
\text { of Holmes' Hole. }\end{array}$ \\
\hline 618 & 15 & Vineyard Sound, off Falmouth:- \\
\hline $\begin{array}{l}619 \\
620\end{array}$ & $\begin{array}{l}15 \\
20\end{array}$ & Vinerard Sound Cuttrhunk \\
\hline & & $\begin{array}{l}\text { Light NW. by N.2 }{ }^{\frac{1}{2}} \text { miles, } \\
\text { Sow and Pigs Light-Ship W. } \\
\text { by } \mathrm{N} \text {. }\end{array}$ \\
\hline
\end{tabular}

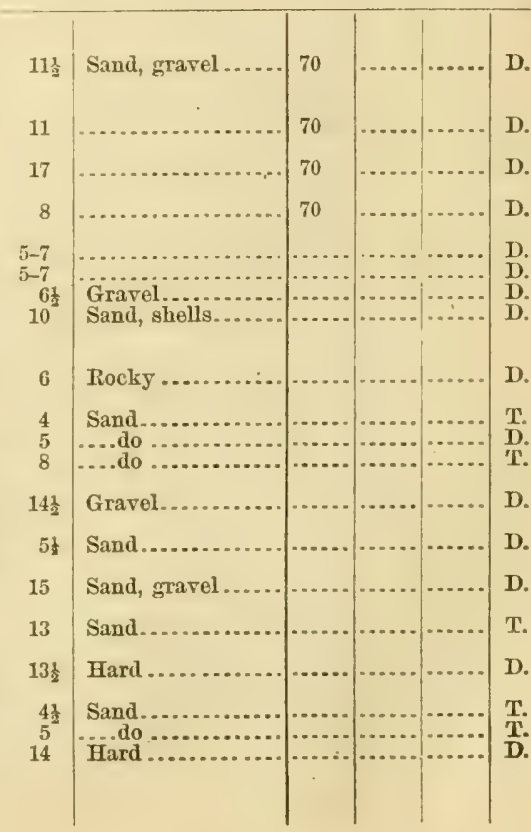


STATIONS FOR 1875-Continued.

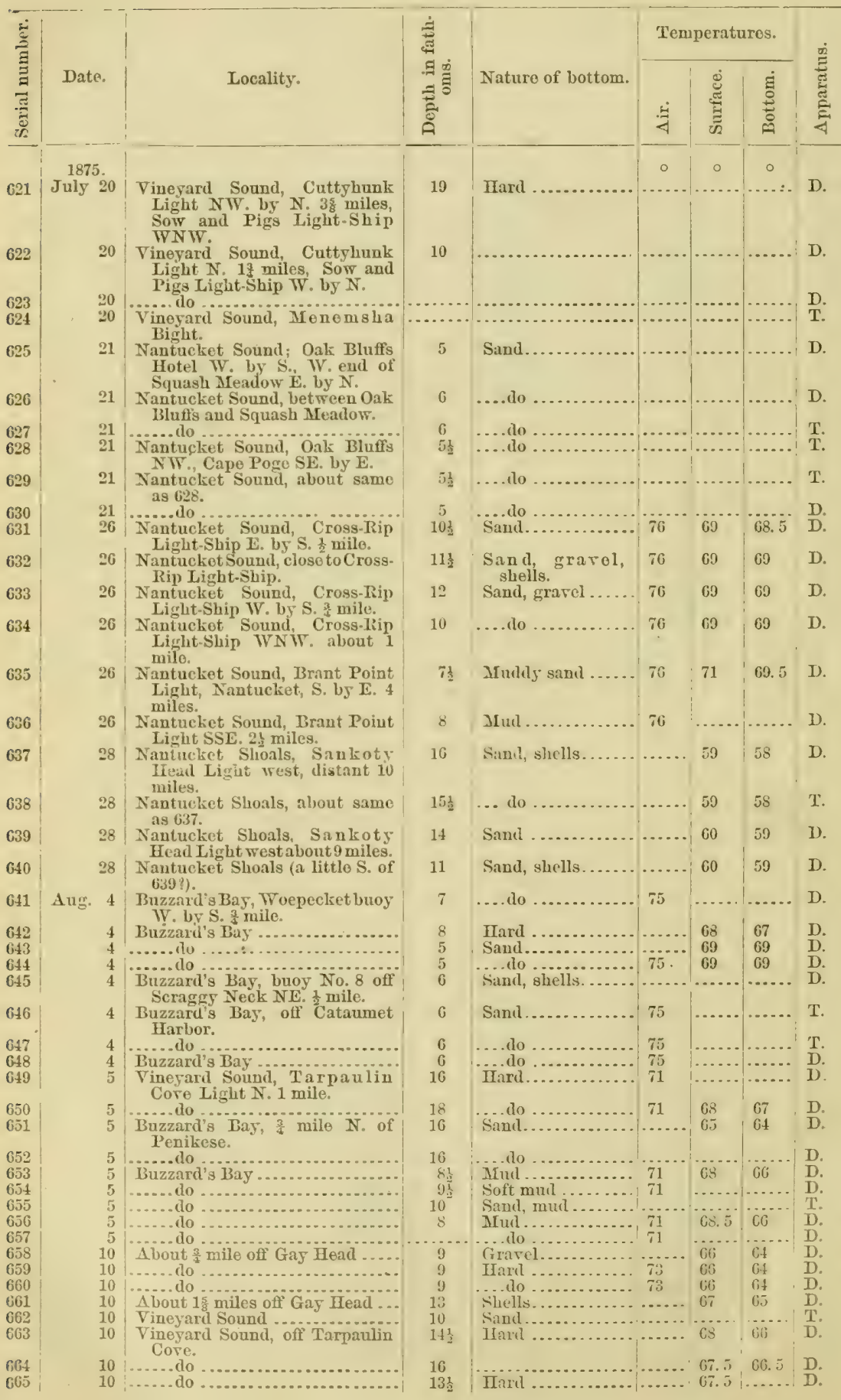


STATIONS HOR 1875-Continued.

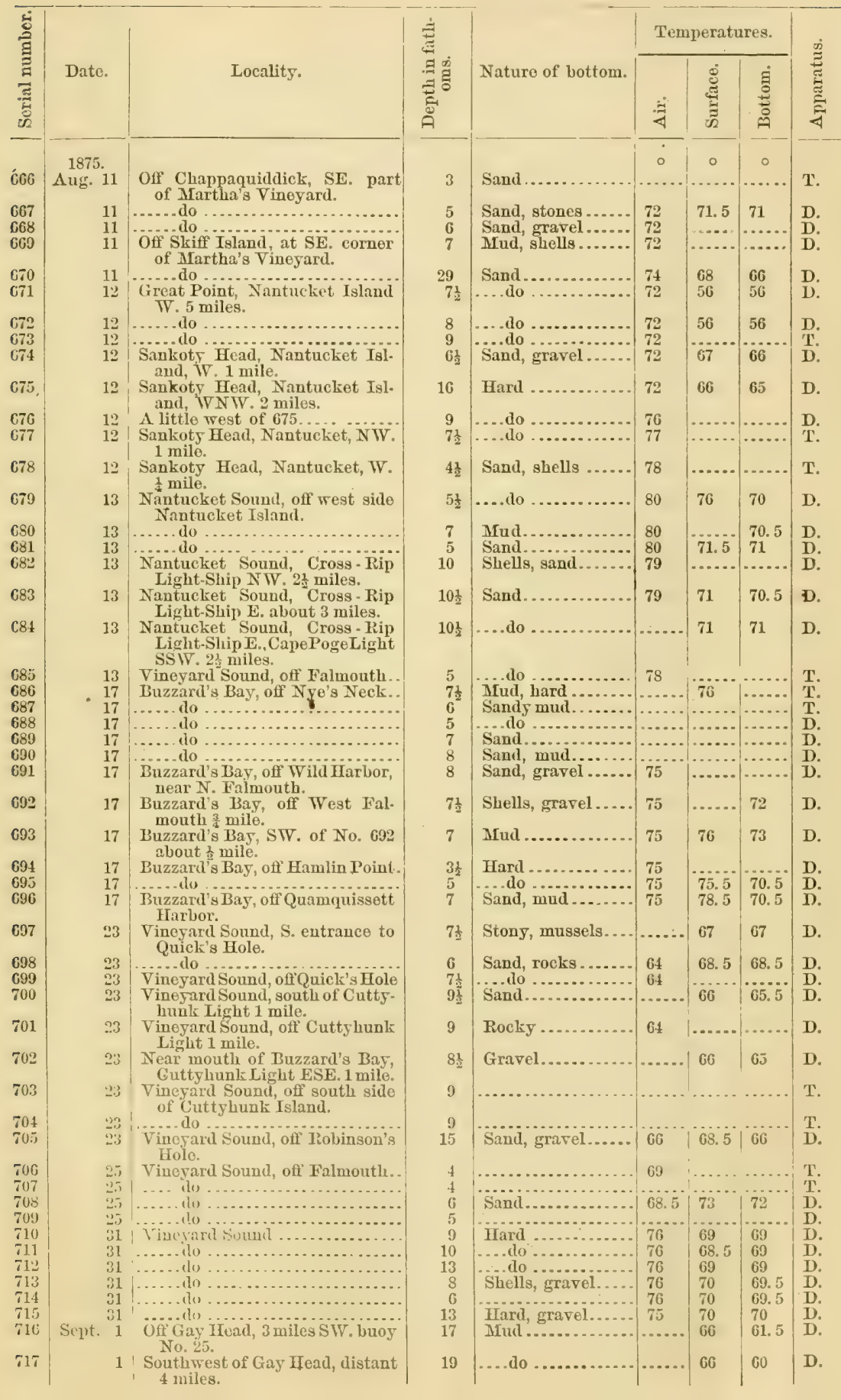


STATIONS FOR 1875-Continued.

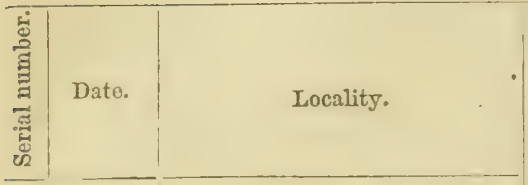

ลี่

1875. 1 Southwegt of Gay Hond, distant 6t miles.

719

1 Southrest of Gay Head, distant 8 miles.

1 Southwest of Gas Head, distant 8 miles.

721

1 Sonthwest of Gay Head, distant 10 miles.

722

72:3
1. Southwest of Gay Iead, clistant 11 miles.

Off NIV. end of Doril's Bridgo licef, Gay Huad.

South of Capo Poge, Martha's Vineyard, in north part of Muskegret Channel.

\section{Alout the same as 725} Äbout 3 miles SE. of Capo Poge. About 4 miles SSE. of Capo Poge. About milo S. of No. $730 \ldots .$. (No record.)

6 Oif Martha's Vinoyarl .......... (No record.)

(i) Off Martha's Vinoyard, 1 miles SE. of Sfuinnocket l'oint.

\section{(No record.)}

\section{......do do}

7 Off Nantucket, milo S. of T'uekernuck Island.

7 Off Nantucket, ott' s. side of Tuckernuek Island.

7

7 ....... dio

(No record.)

8 miles S. of Groat Roumel Shoal buoy, 6.2 miles a little $\mathrm{NE}$. of Great Point, Nantucket.

83 miles SE. of Great Round Shoal buoy, 9 miles from Great Point.

84 miles SSE. of Great Round Shoal bno, 9 miles from Great Point.

8 Off Nantucket, X. of McBlair's Shoal.

8 ...... (10

8 ........ (l)

158 miles east of Great Point, Nantucket.

1.5 9 miles east of Great Point, Nantucket.

158 miles east of Great Point, Nan. tucket.

159 miles east of Great Point, Nantricket.

1511 miles east of Great Point Nantucket.

1512 miles east of Great Point, Nantucket.

1515 miles east of Great Point, Nantucket.

1515 miles east of Great Point, Nantucket.

$1516 \frac{1}{2}$ miles east of Great Point, Nantucket.

$157 \frac{1}{2}$ miles east of Great Point, Nantucket.

157 miles east of Great Point, Nan. tucket.

$157 \frac{1}{2}$ miles east of Great Point, Nantucket.

20 Off Southwest Ledge, Gay Head NE. $11 \frac{1}{2}$ miles.
8 ......do do - of

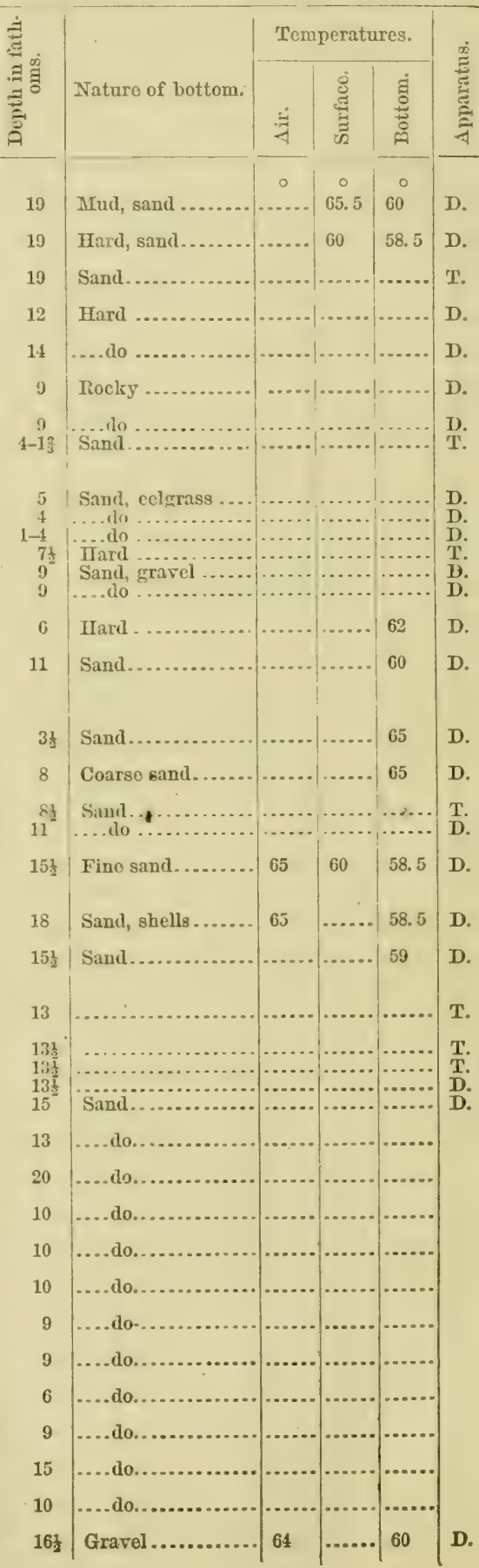


STATIONS FOR 1875-Concluded.

\begin{tabular}{|c|c|c|c|c|c|c|c|c|}
\hline \multirow{2}{*}{ 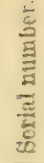 } & \multirow[b]{2}{*}{ Date. } & \multirow{2}{*}{ Locality. } & \multirow{2}{*}{ 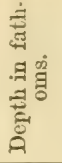 } & \multirow[b]{2}{*}{ Nature of bottom. } & \multicolumn{3}{|c|}{ Temperatures. } & \multirow[b]{2}{*}{ 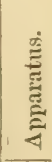 } \\
\hline & & & & & $\frac{\dot{3}}{4}$ & 总 & 节 & \\
\hline$a n$ & $\begin{array}{l}1875 . \\
\text { Sicpt. } 20\end{array}$ & $\begin{array}{l}\text { Off Southwest Ledge, } \frac{1}{2} \text { mile } \mathrm{W} \text {. } \\
\text { of } 762 \text {. }\end{array}$ & 17 & Gravel, sant . & $64^{\circ}$ & o & $60^{\circ}$ & D. \\
\hline 761 & 20 & $\begin{array}{l}\text { Off Southwest Ledge, } \frac{x}{3} \text { mile S. } \\
\text { of } 76 \% \text {. }\end{array}$ & & & & & & \\
\hline 705 & 20 & $\begin{array}{l}\text { Off Southwest Ledge, } \frac{3}{2} \text { mile Wr. } \\
\text { of } 763 \text {. }\end{array}$ & 17 & Sand, grarel. & & & 60 & D. \\
\hline 766 & 20 & $\begin{array}{l}\text { On Southrwest Ledge, } \frac{1}{4} \text { mile NW. } \\
\text { of } 765 \text {. }\end{array}$ & 17 & .....do . & …s. & & 60 & D. \\
\hline 707 & 20 & Off Southrest Ledge, $1 \frac{1}{4}$ miles & 18 & Sand.... & 64 & & 61 & D. \\
\hline $\begin{array}{l}765 \\
762\end{array}$ & $\begin{array}{l}20 \\
20\end{array}$ & $\begin{array}{l}9 \text { miles SW. of Gay Head } \\
6 \text { miles SW. } \frac{3}{3} \text { W. of Gay Head. }\end{array}$ & $\begin{array}{l}20 \\
20\end{array}$ & 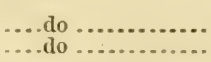 & 64 & ... & 61 & D. \\
\hline
\end{tabular}

STATIONS FOR 18\%7, 1878, AND 1879, WITH HEADQUARTERS AT SALEM, MASS., HALIFAX, N. S., GLOUCESTER AND PROVINCETOWN, MASS.

During these three years the dredgings were carried on from the U. S. Str. Speedwell, commanded in 1877 by Lient. Commander A. G. KelIogg, in 1878 by Lient. Commander L. A. Beardslee, and in 1879 by Lieut. Z. L. Tamner. In 1877, headquarter's were first established at Salem, and the stations male from there covered the northern part of Massachusetts Bay, and portions of the Gulf of Maine, off Cape Ann. During the session of the commission of arbitration on the fishery claims, however, the hearlquirters were removed to Halifax, N. S., and dredgings were made in the water's off that coast, from the last of August to the first of October. The Speedwell also made a line of stations on her trip across the Gulf of Maine, from Cape Anu to Cape Sable, N. S. In 1878, with headquarter's at Gloneester, Mrass., the area dredged orer included the northern and central parts of Massachusetts Bay, and the Gulf of Maine, off Cape Ann. In 1s79, the dredging grounds were the sonthern part of Massachusetts Bay, and the Gulf of Maine, off' Cape Corl. The bottom temperatures in 1877 were mostly taken with Ailler-Casella self-registering, deep-sea thermometers, but in 1878 and 1879 NegrettiZizmbia thermometers were used for that purpose. All the temperatures for 1869 were taken with more than usual care, the thermometers emploxed being frequently compared with a reliable standard. 


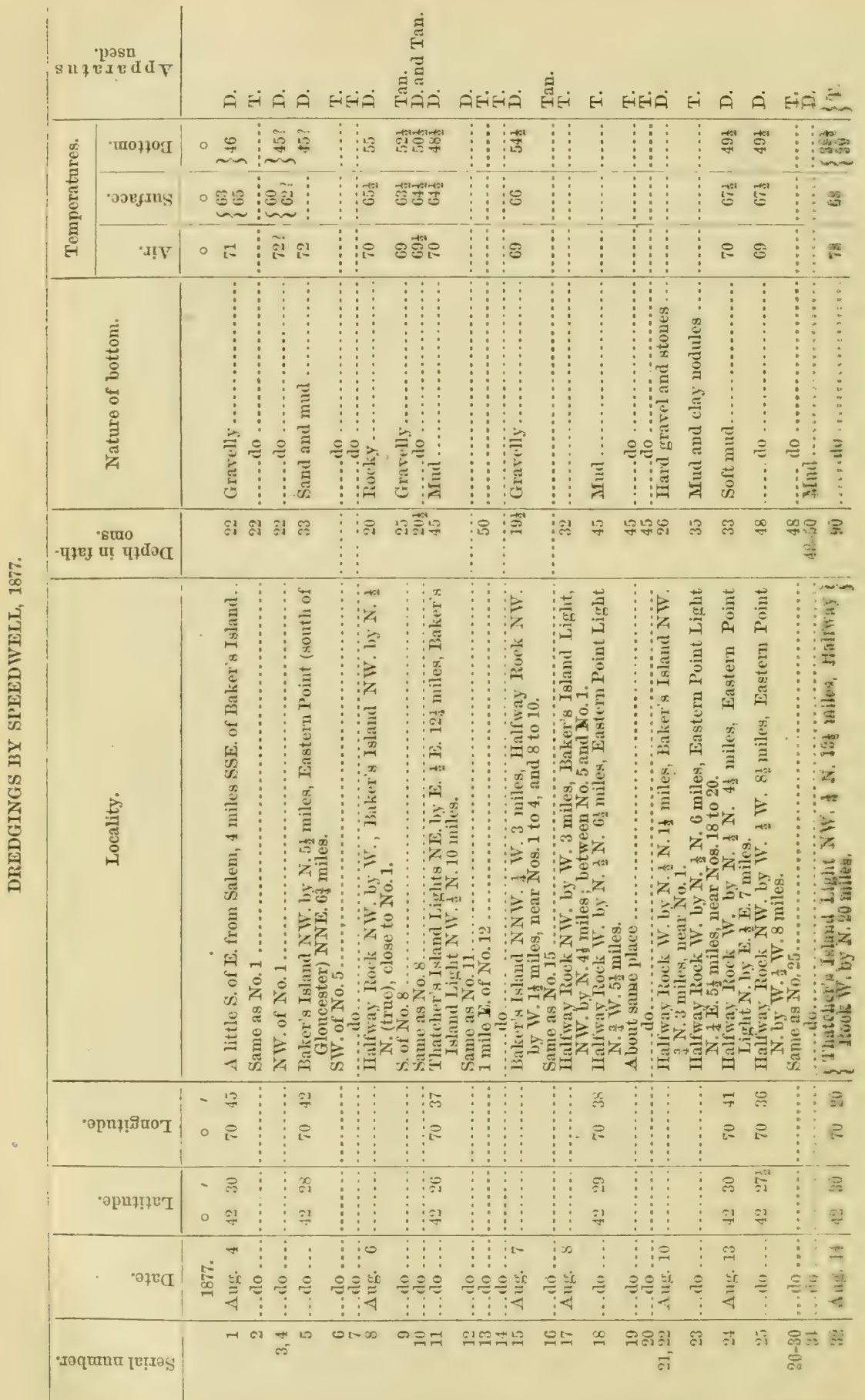




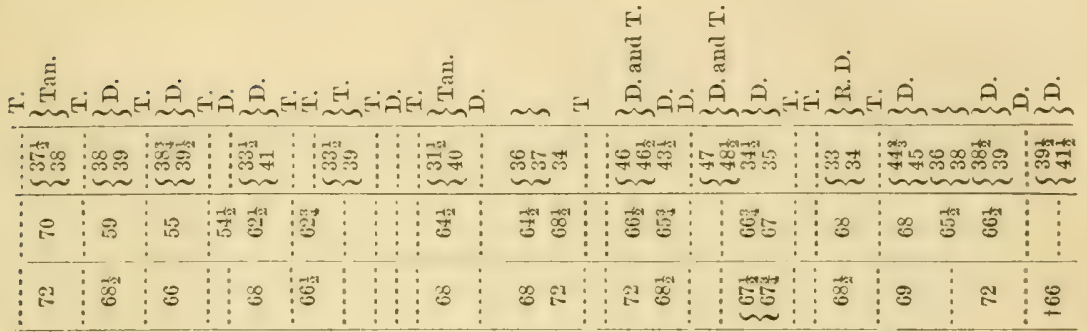

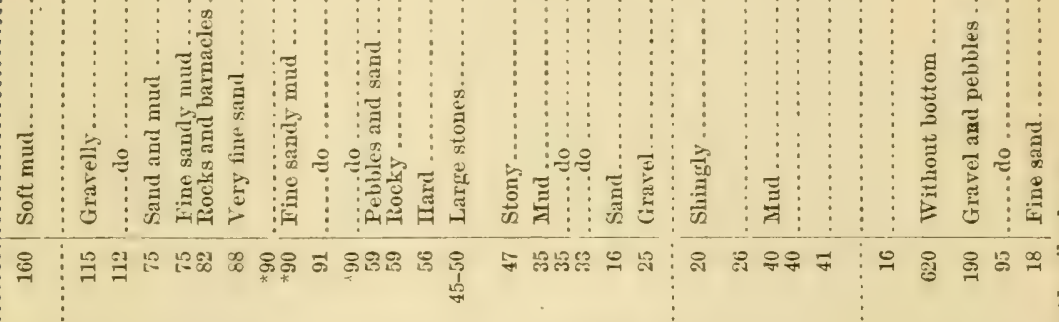

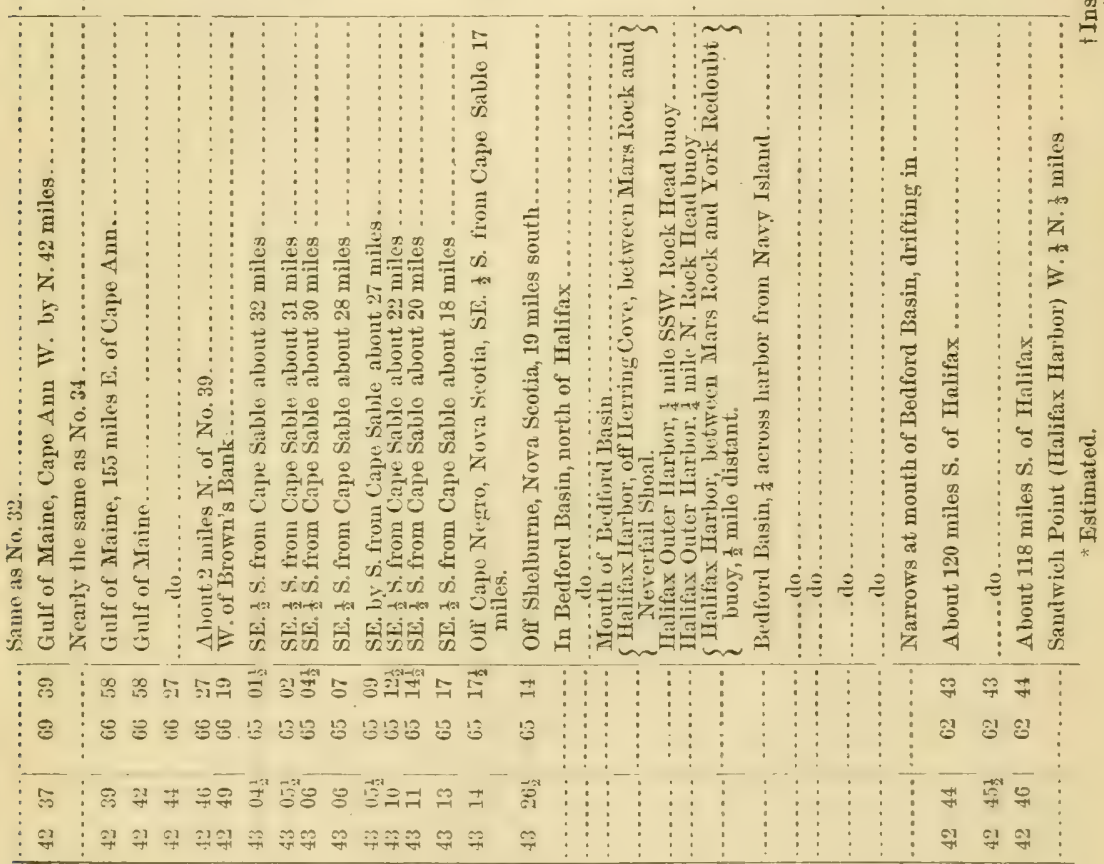

i $=$

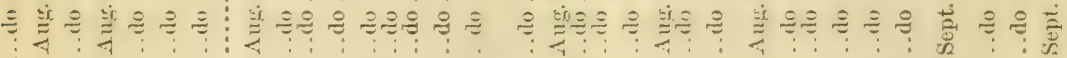




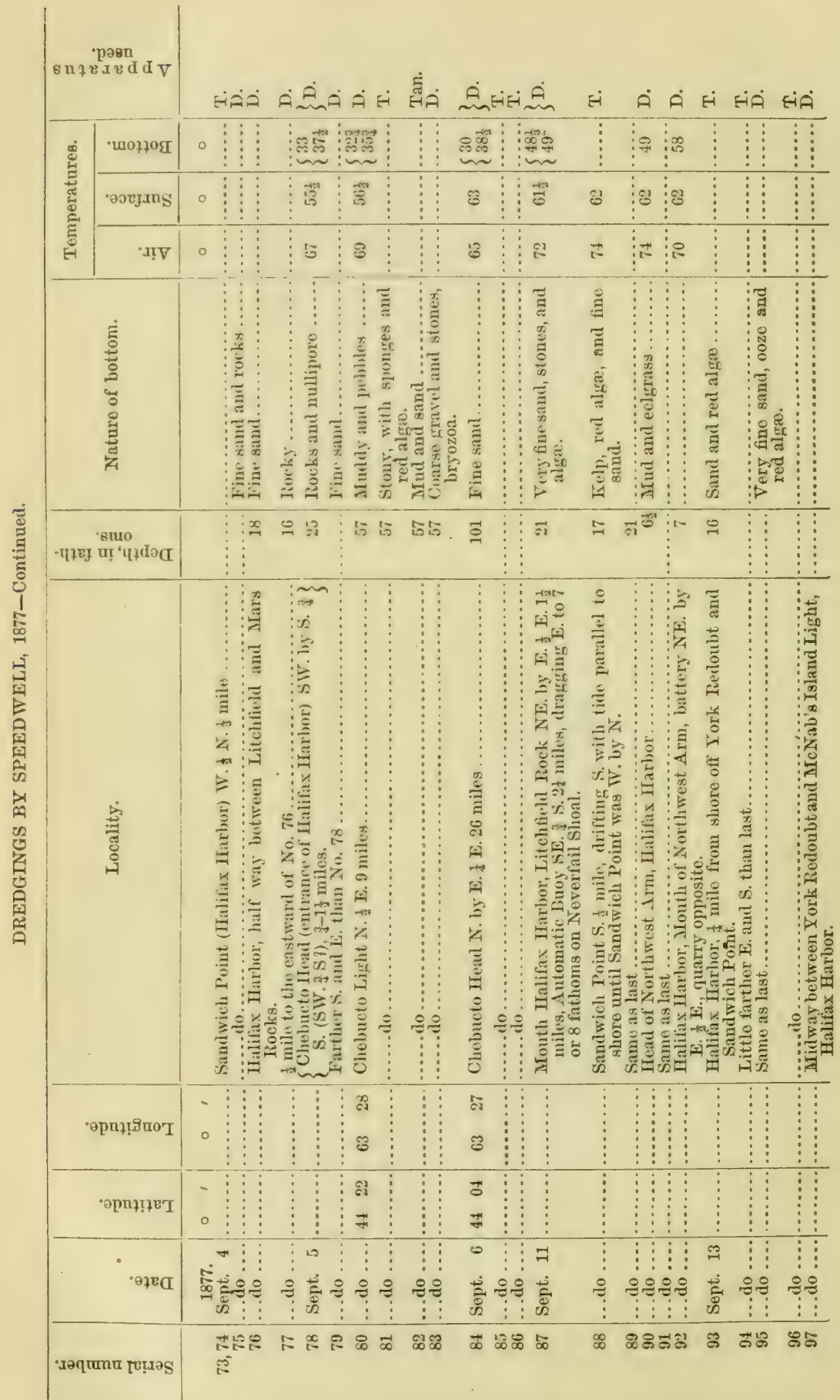




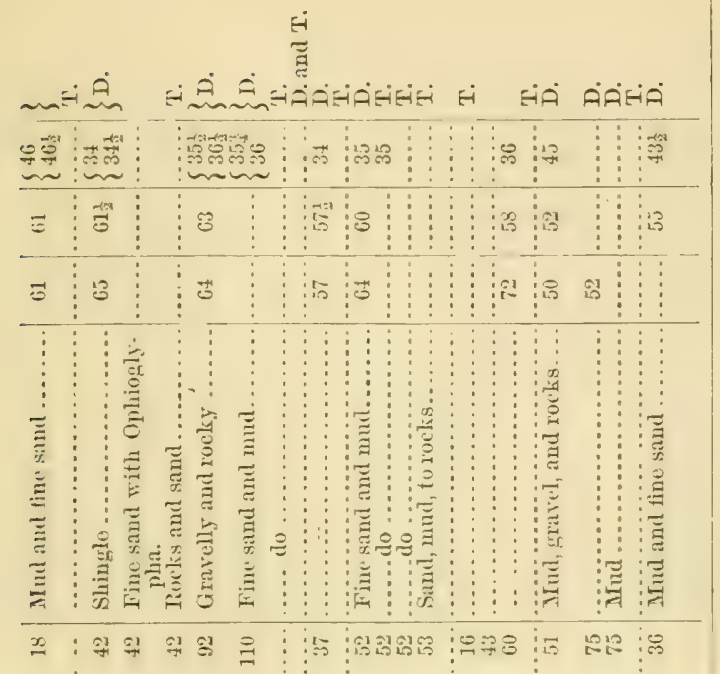

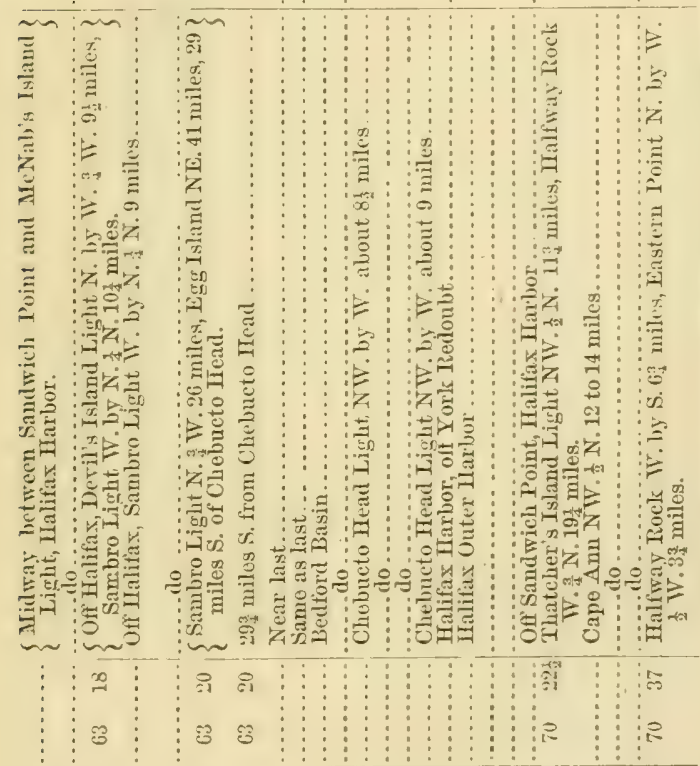

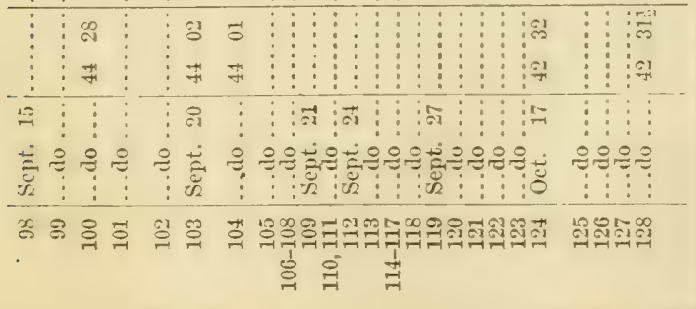

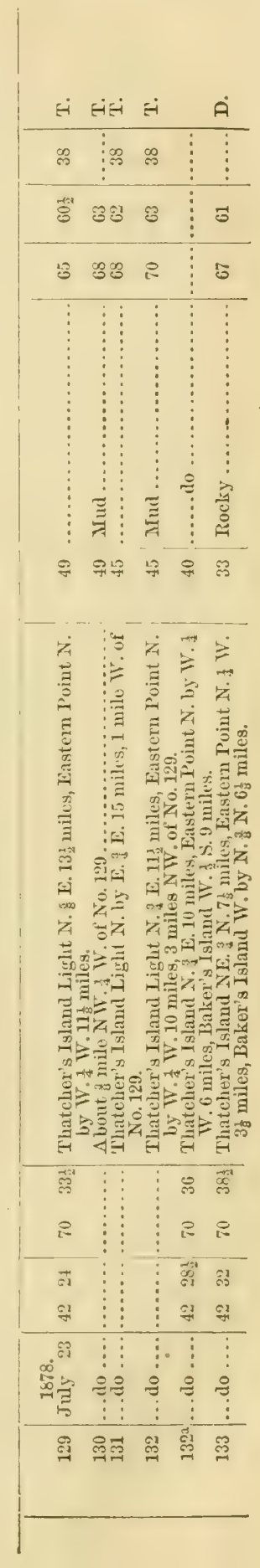


30 REPOR' OF COMMISSIONTR OF FISH AND FISHERIES.

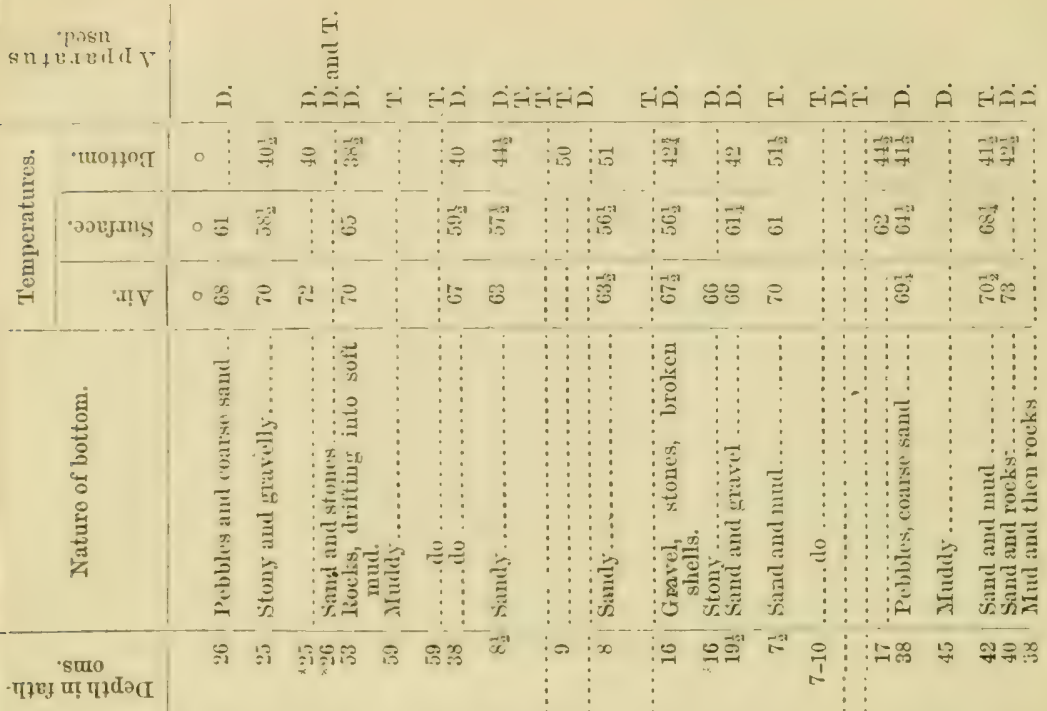

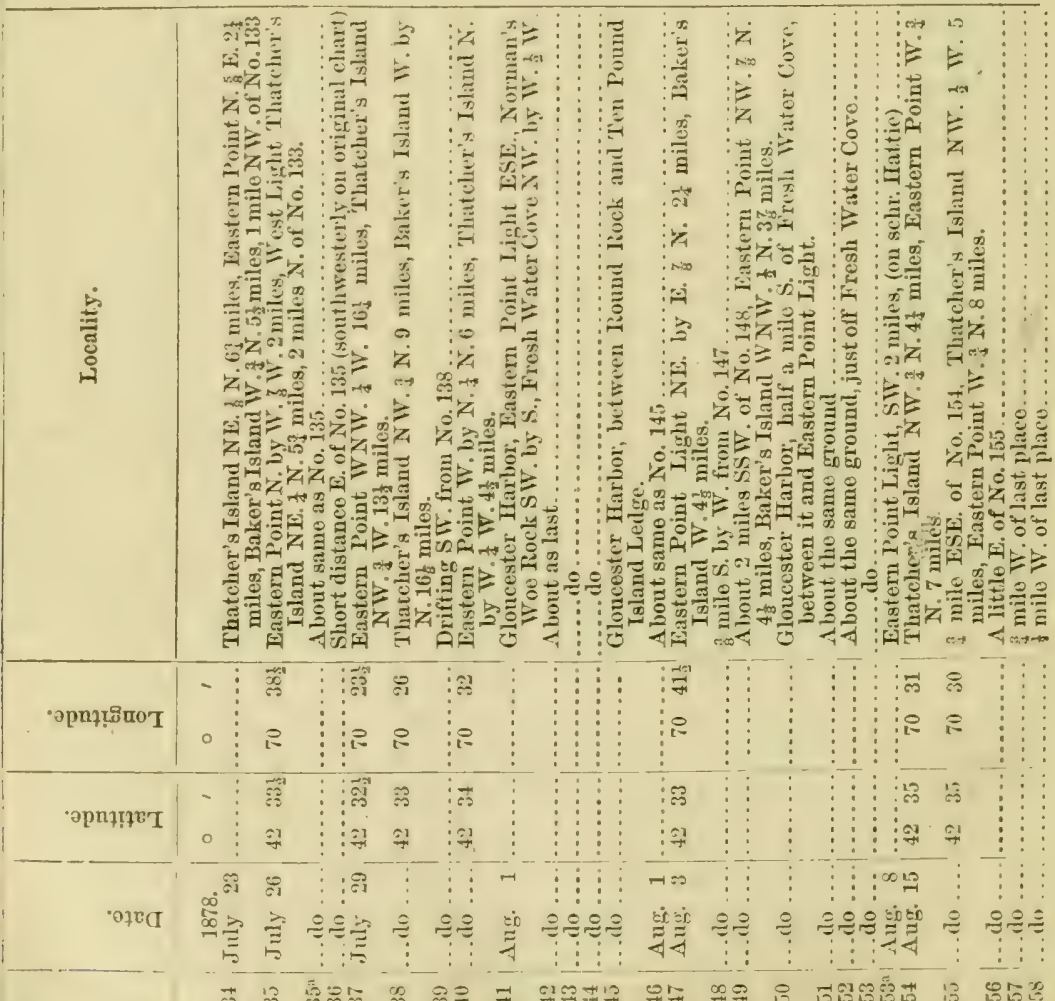

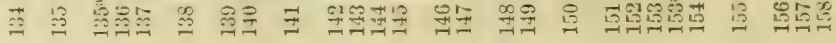




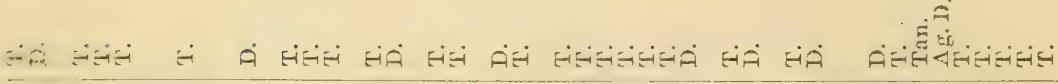

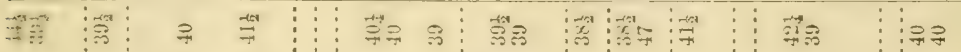

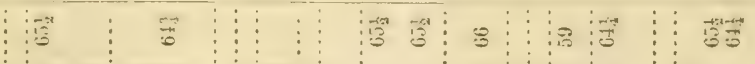

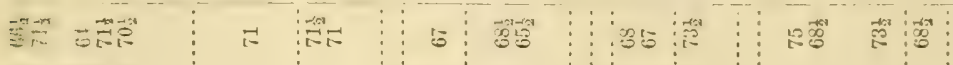

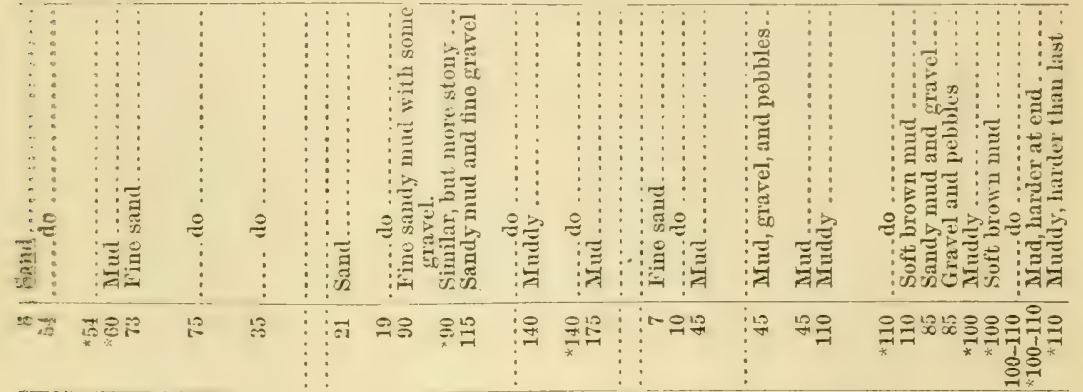

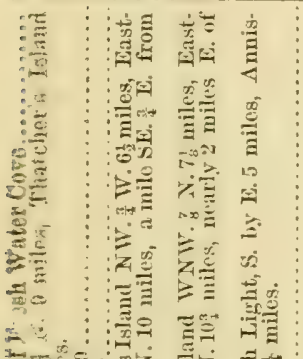

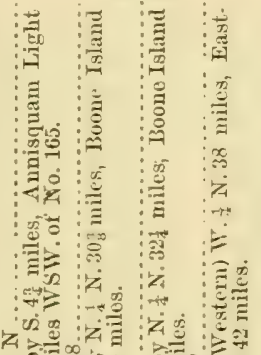

क्य

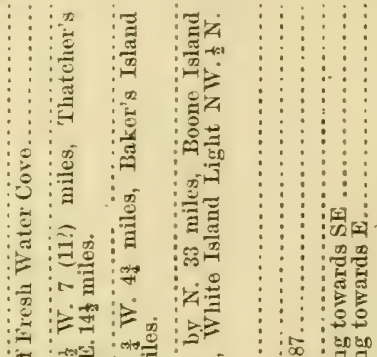

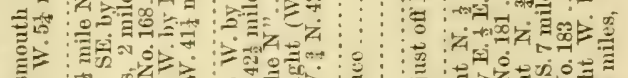

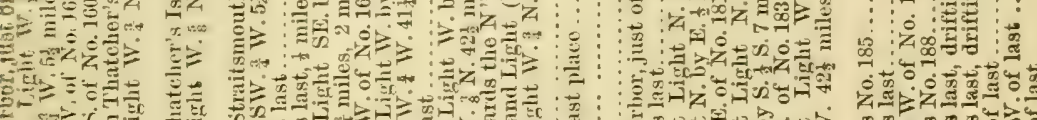
过

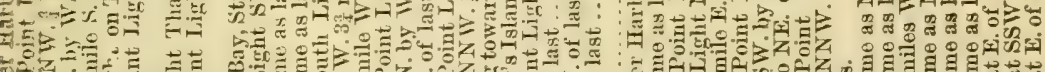

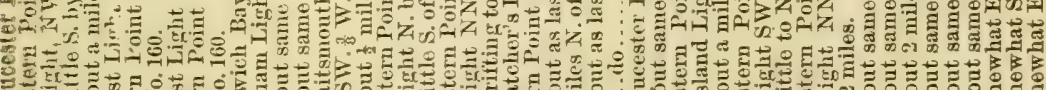

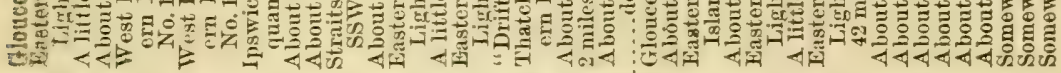

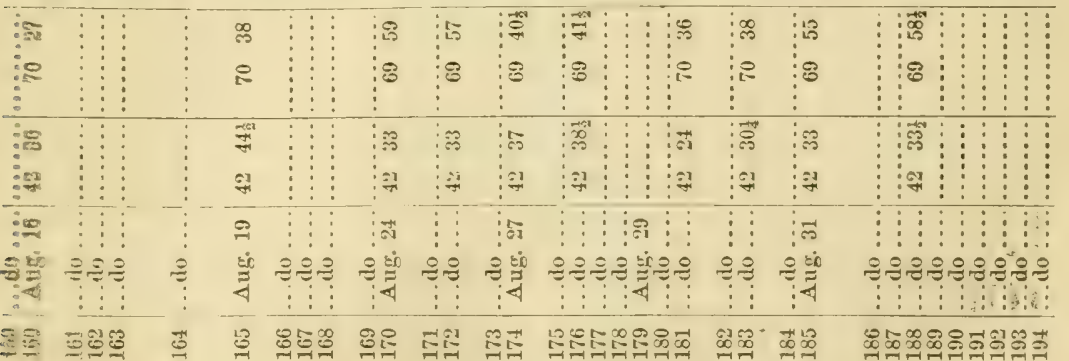




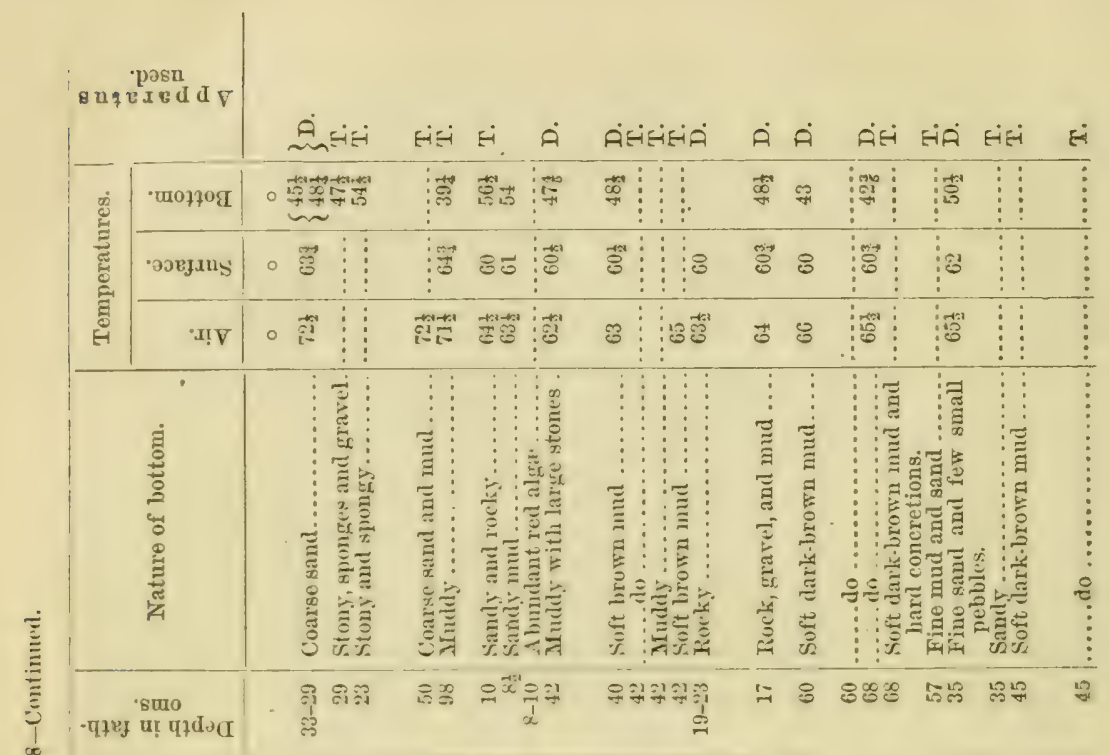

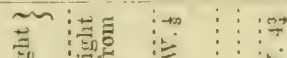

ำ

ह $\vdots$ 㝵

总

क

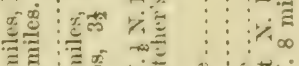

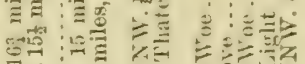

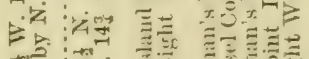

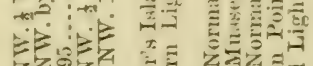

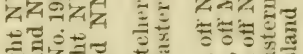

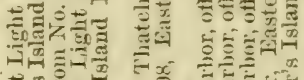

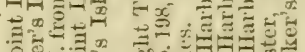

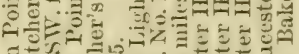

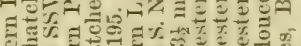

कृ

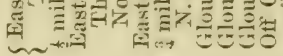

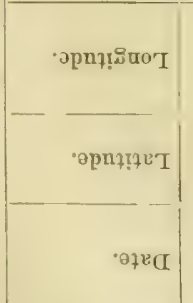

๑)

\& $\vdots$

8

$\vdots:=$

$\vdots 0$

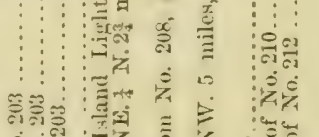

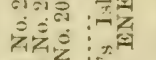

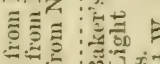

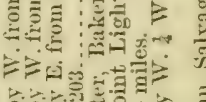

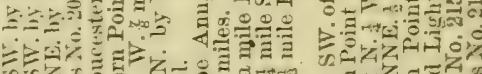

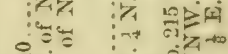

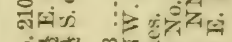

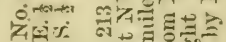

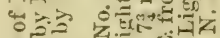

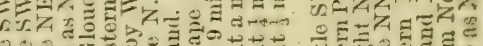

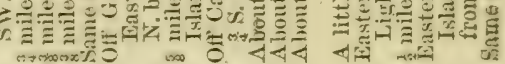
$\vdots \vdots \vdots \vdots$

$\vdots \vec{x}$

8

औ $\vdots \vdots \vdots \vdots$

$\rightarrow$ is

$8 \quad \vdots=$

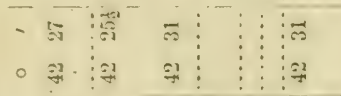

$\vdots \vdots \vdots \vdots 0$

$$
6
$$

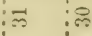

원

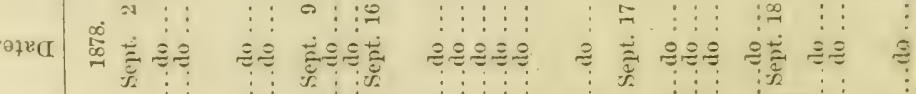

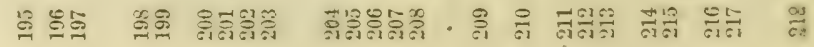




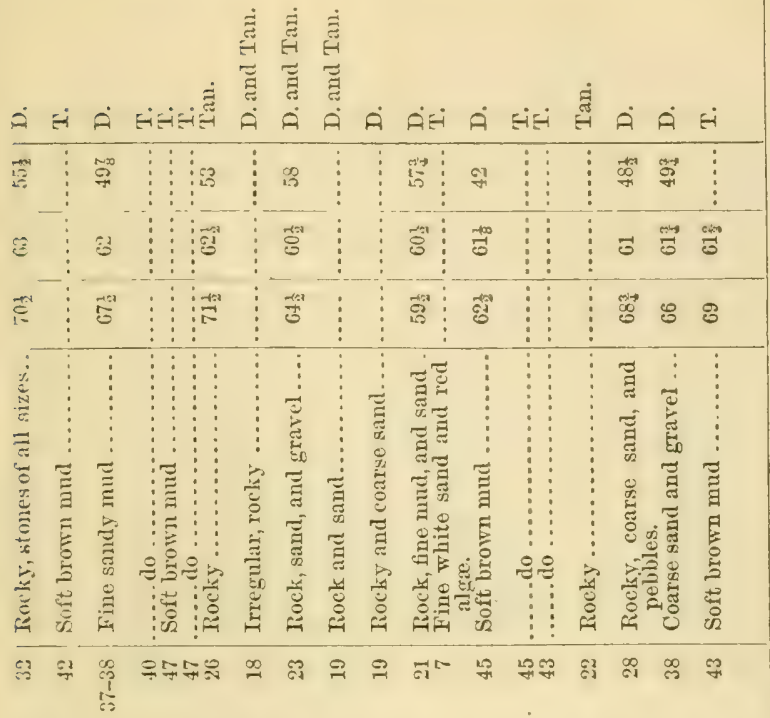

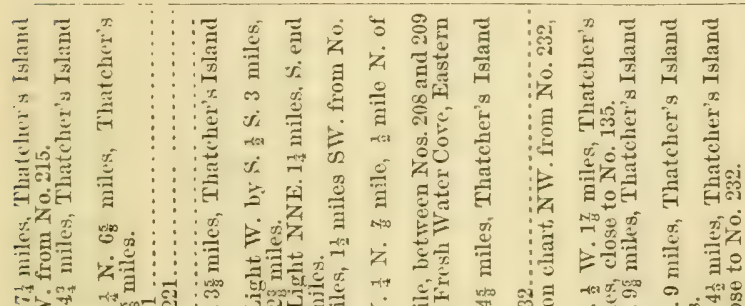
ron mon 0

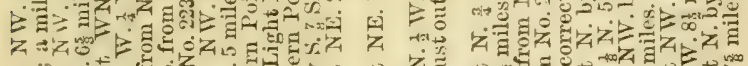

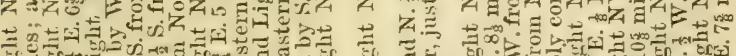
Ex Ex-

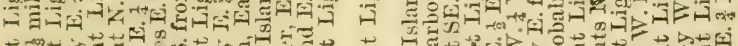

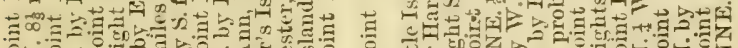

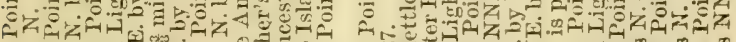

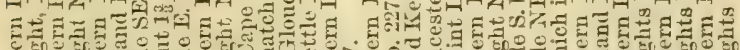

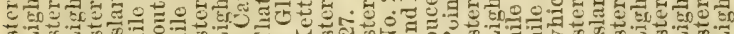

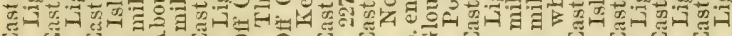

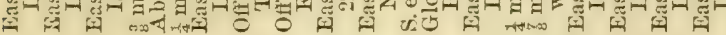

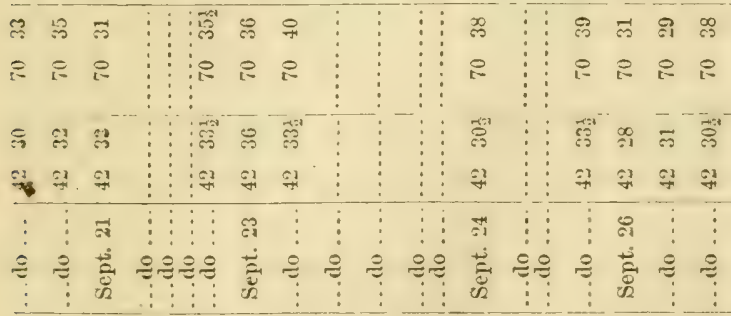

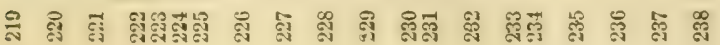

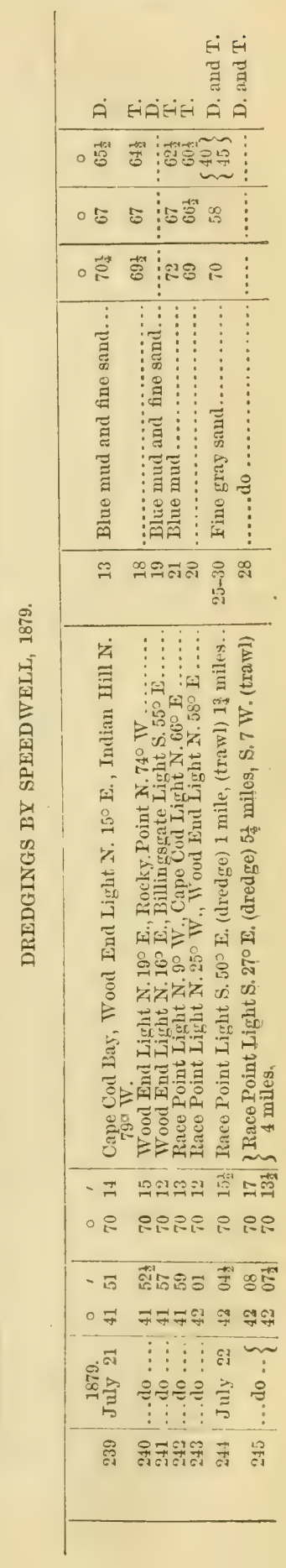


34 REPORT OF COMMISSIONER OF FISH AND FISHERIES.

'posu

$8 n 78 x ข d d \bar{T}$

\begin{tabular}{|c|c|c|c|c|c|}
\hline \multirow{3}{*}{ 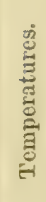 } & \multirow{2}{*}{ 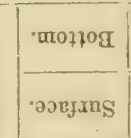 } & \multicolumn{4}{|c|}{ 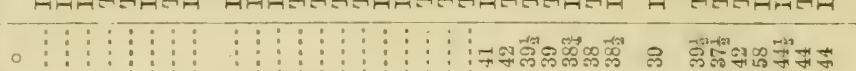 } \\
\hline & & 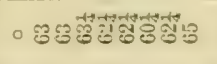 & タ8タむむい: & 舫 & 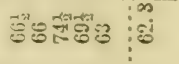 \\
\hline & 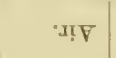 & 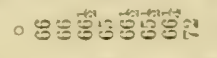 & 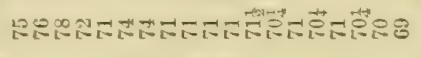 & 窝 & 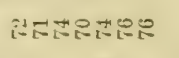 \\
\hline & 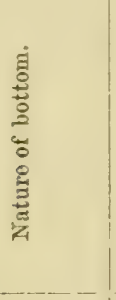 & 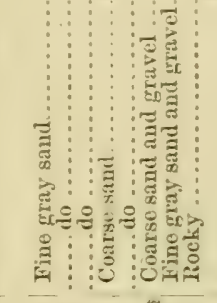 & 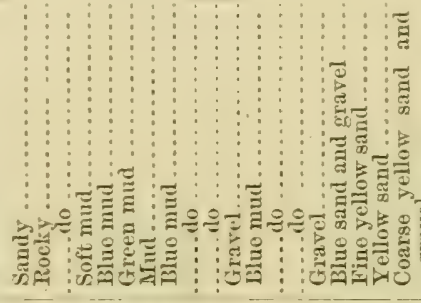 & 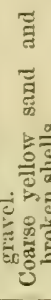 & 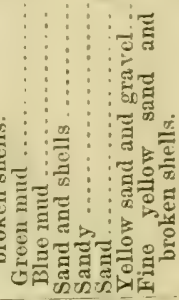 \\
\hline -тұ्म & 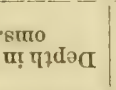 & 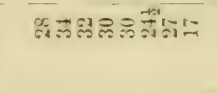 & 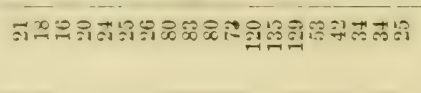 & $\circ$ & 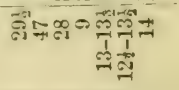 \\
\hline \multicolumn{2}{|r|}{ 宅 } & 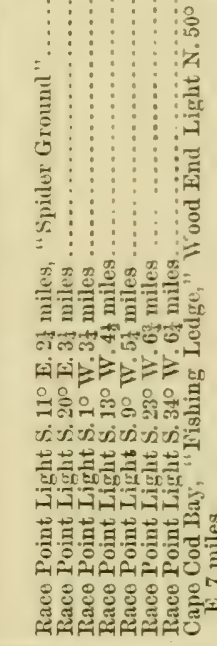 & 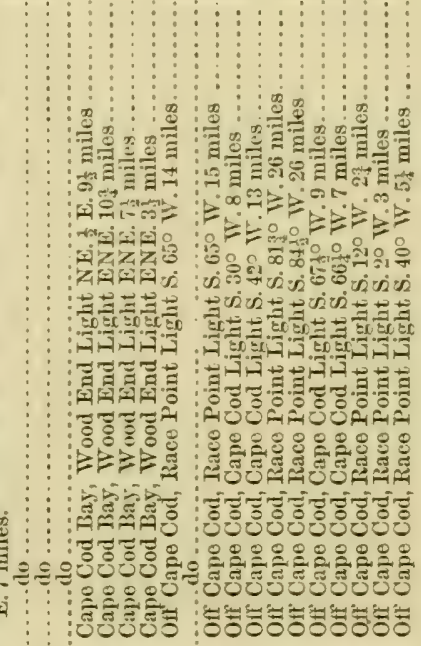 & 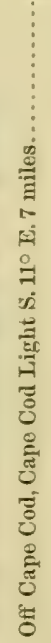 & 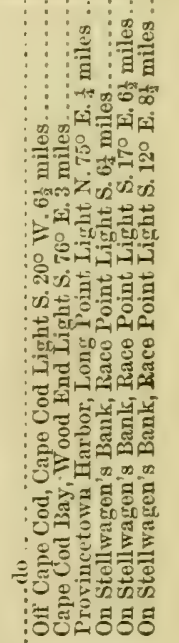 \\
\hline \multicolumn{2}{|c|}{ 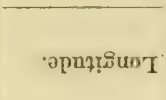 } & 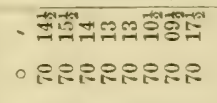 & : & s & :8: \\
\hline \multicolumn{2}{|r|}{ •өри!юет } & 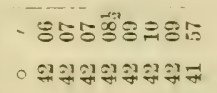 & 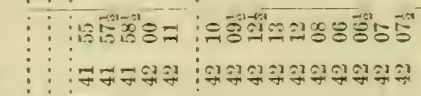 & 8 & 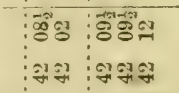 \\
\hline & $\cdot$ วาв & 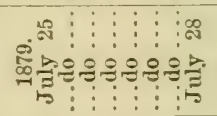 & 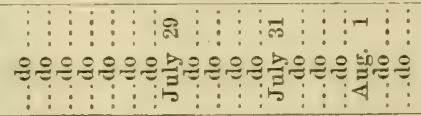 & $\stackrel{\vdots}{\vdots}$ & 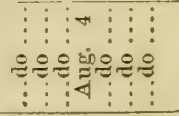 \\
\hline \multicolumn{2}{|c|}{ 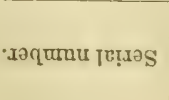 } & 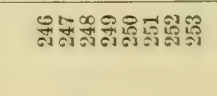 & 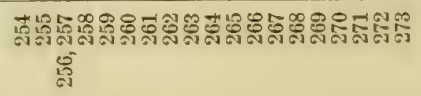 & $\underset{5}{\pi}$ & 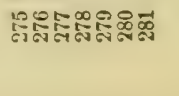 \\
\hline
\end{tabular}


Dीं

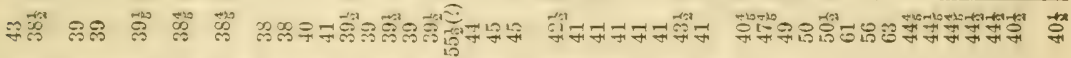
ปี่

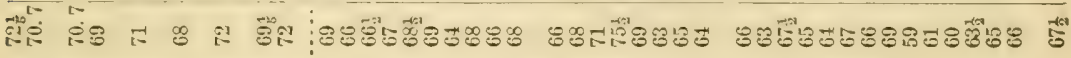

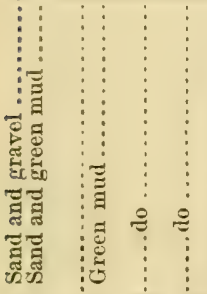

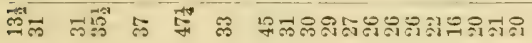

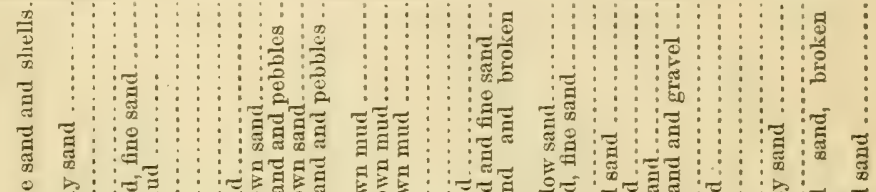

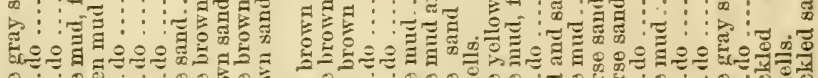
焉

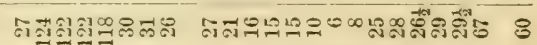

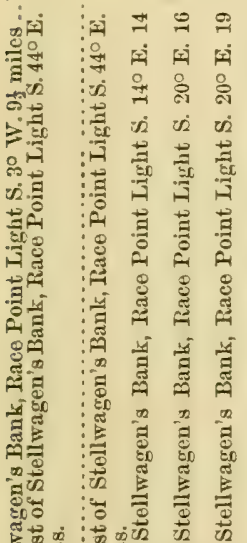

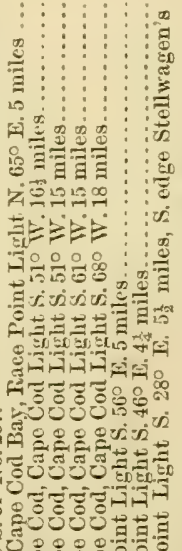

20.9 co

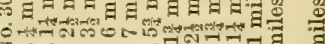

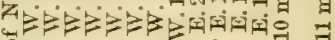

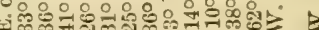

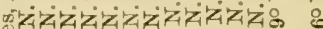

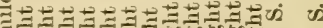

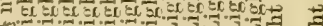

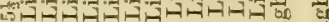
งอง

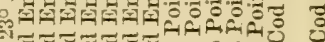

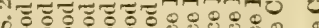
20

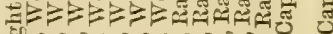
उ.

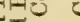

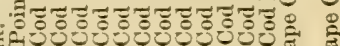

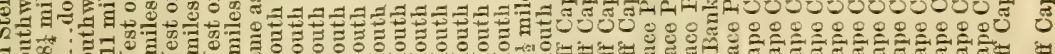

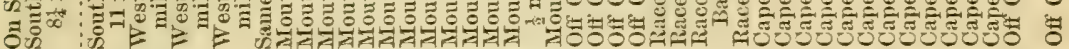

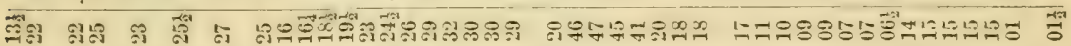

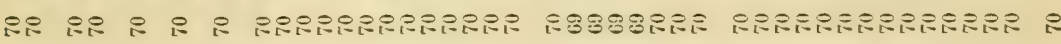

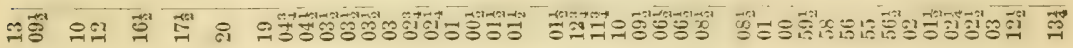

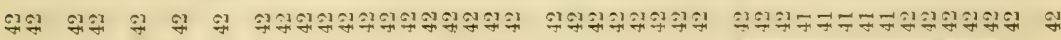

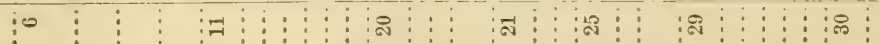

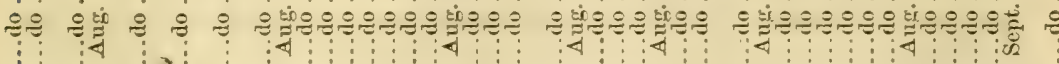
\$ 


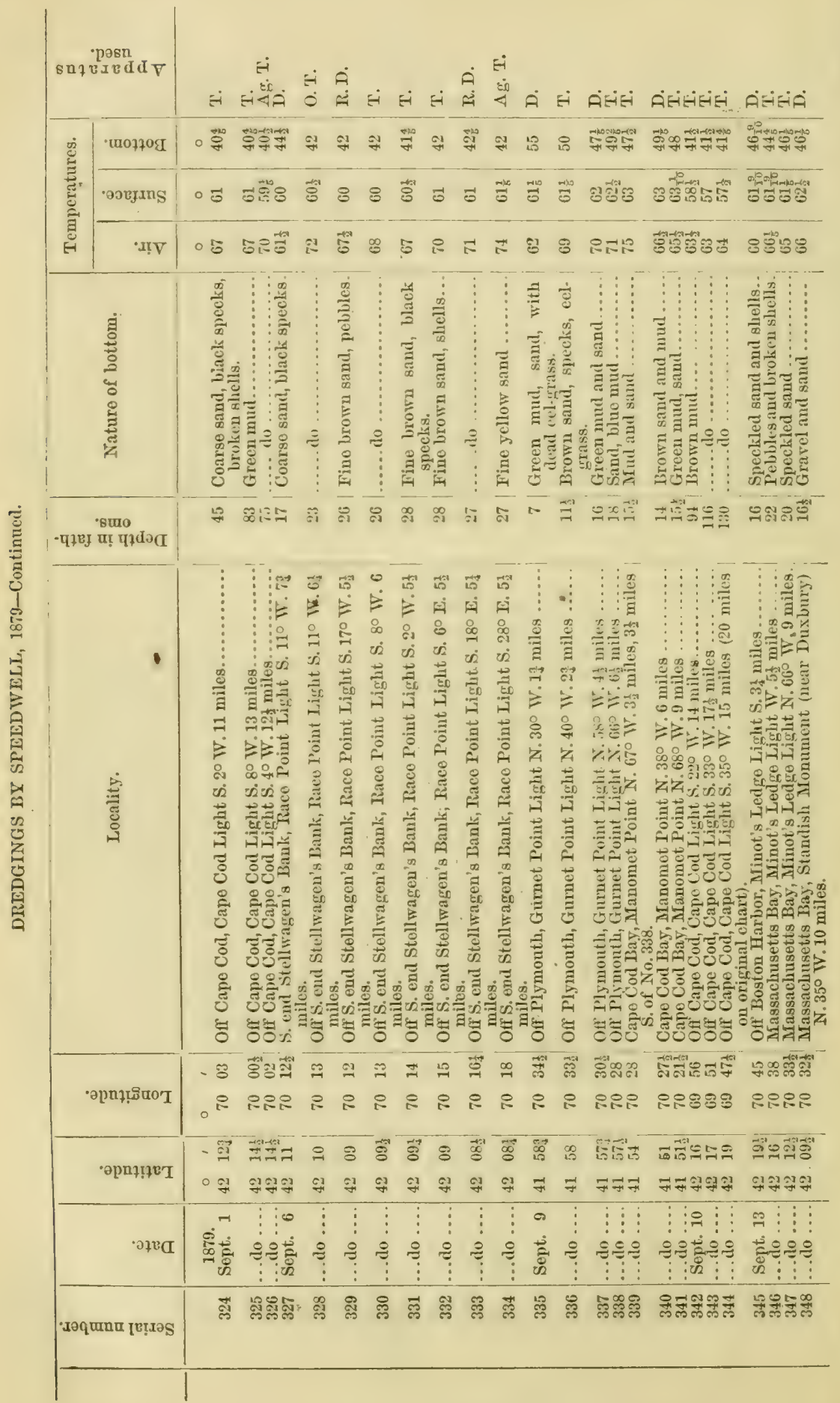


Bं

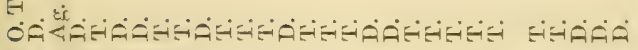

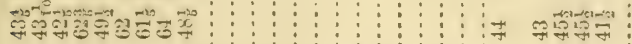

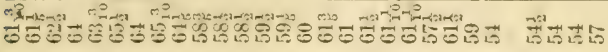

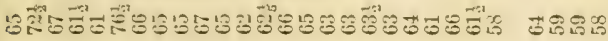

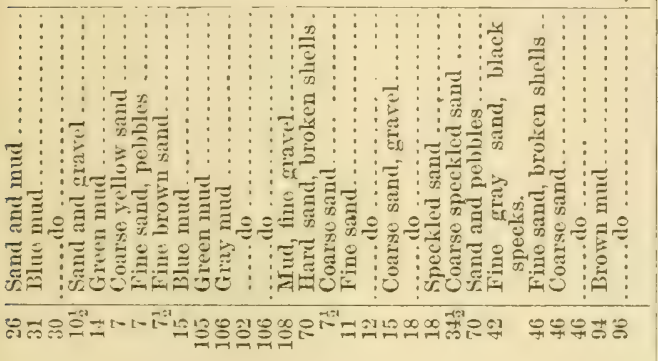

\begin{tabular}{c}
$\vdots$ \\
$\vdots$ \\
$\vdots$ \\
$\vdots$ \\
$\vdots$ \\
$\vdots$ \\
\hdashline
\end{tabular}

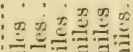

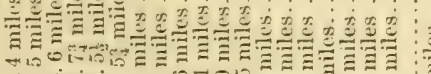

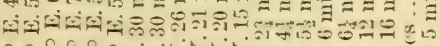

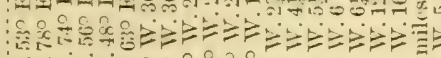

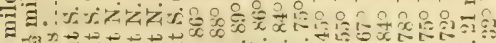

э第

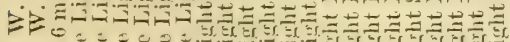

$800-000$

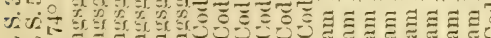

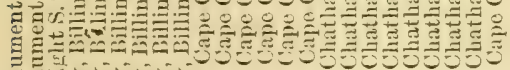

苛

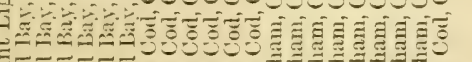

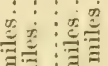

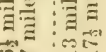

Ora

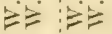

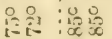

sis

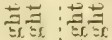

ラこ

כัซ

-

气.

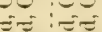

वें

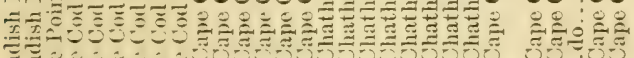

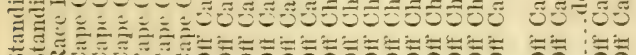

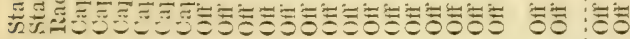

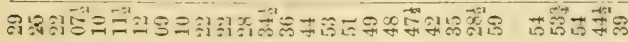

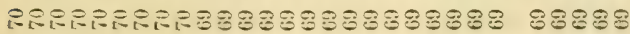

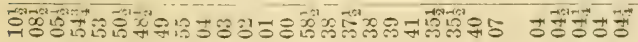

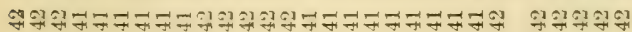

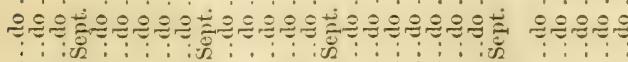

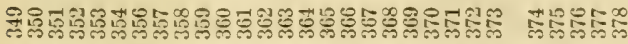
跑 


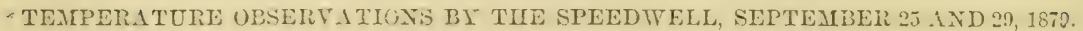

\begin{tabular}{|c|c|c|c|c|c|c|}
\hline \multirow{2}{*}{ 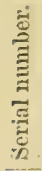 } & & \multirow[b]{2}{*}{ Locality. } & \multirow{2}{*}{ 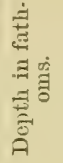 } & \multicolumn{3}{|c|}{ Temperatures. } \\
\hline & 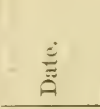 & & & $\overrightarrow{\bar{z}}$ & 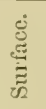 & $\frac{\tilde{E}}{\frac{E}{0}}$ \\
\hline 1 & $\begin{array}{l}1879 . \\
\text { Sevt. } 25\end{array}$ & Long Point WVNT 3 mile. & & 0 & ${ }^{0}$ & 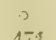 \\
\hline$\frac{1}{2}$ & & Wood Emi Light ITI $^{\frac{1}{4}}$ nile. & 21 & $\ldots$ & 5.94 & $4 \pi^{8}$ \\
\hline 3 & 29 & IVood End Light XE. $\frac{1}{4}$ mile. . . . . . . & 22 & $\ldots$ & 5 & 4 \\
\hline 4 & 29 & Wood End Light N. $80^{\circ}$ E. 2 miles . & $24 \frac{1}{2}$ & $\ldots$ & 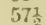 & $44^{7}$ \\
\hline 5 & 29 & 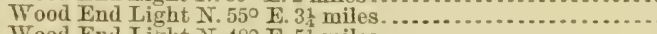 & $21^{2}$ & ....... & $57 \frac{1}{5}$ & $45^{5}$ \\
\hline 6 & 29 & 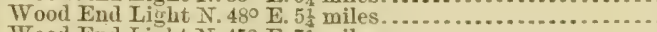 & 21 & $\ldots . .$. & $57 \frac{1}{2}$ & $45 \frac{1}{5}$ \\
\hline 7 & 29 & 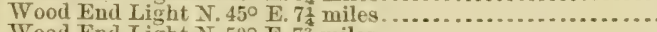 & $19 \frac{1}{2}$ & ....... & $58 \frac{1}{2}$ & $46_{15}^{5}$ \\
\hline 8 & 29 & 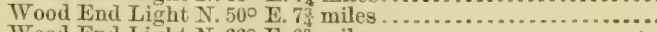 & $19 \frac{1}{2}$ & ....... & $58 \frac{1}{2}$ & 46 \\
\hline 9 & 29 & 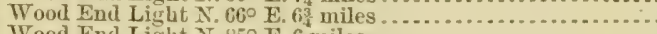 & 24 & & $59 \frac{1}{5}$ & $44 \frac{1}{5}$ \\
\hline 10 & 29 & 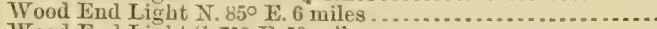 & 26 & 691 & $58^{\circ}$ & $44^{\circ}$ \\
\hline 11 & 29 & 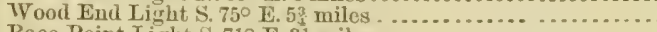 & 30 & $69 \frac{1}{2}$ & $58 \frac{1}{2}$ & 44 \\
\hline 12 & 29 & Race Point Light S. $71^{\circ}$ E. $3^{1}$ miles ..... & 33 & $66^{2}$ & & 44 \\
\hline 13 & \pm 9 & 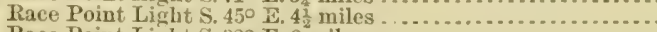 & 34 & 66 & 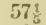 & $44 \frac{1}{5}-100$ \\
\hline 14 & 29 & 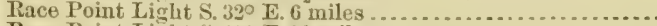 & 11 & 67 & $58^{\circ}$ & $513^{3}$ \\
\hline 15 & 29 & 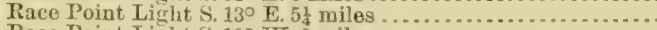 & 23 & $63 \frac{1}{2}$ & $57 \frac{1}{6}$ & $52 \frac{2}{2}$ \\
\hline 16 & 29 & 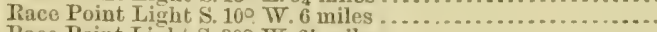 & 19 & $62 \frac{1}{2}$ & 58 & 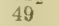 \\
\hline 17 & 29 & 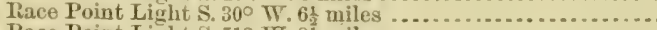 & 26 & 63 & 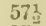 & $46 \frac{1}{2}$ \\
\hline 18 & 29 & Race Point Liglut S. $51^{\circ}$ W. $8 \frac{1}{2}$ miles .... & 28 & $62 \frac{1}{2}$ & 57 & $45^{\circ}$ \\
\hline 19 & 29 & Race Point Light S. $58^{\circ}$ W. 93 miles .... & 49 & $62 \frac{1}{5}$ & $57 \frac{4}{5}$ & $43 \frac{7}{6}$ \\
\hline 20 & 29 & Race Point Light S. $70^{\circ} \mathrm{W}$. $9 \frac{1}{3}$ miles .... & 32 & 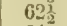 & 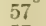 & 44 \\
\hline 21 & 29 & Race Point Light S. $66^{\circ}$ TV. 5 miles .... & 21 & $64^{2}$ & 57 & 45 \\
\hline 22 & 29 & 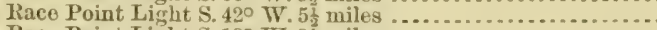 & 28 & $62 \frac{1}{6}$ & 564 & 46 \\
\hline 23 & 29 & Race Point Light S. $18^{\circ}$ W. $2 \frac{1}{2}$ miles ... & 33 & ..... & $57 \frac{1}{2}$ & 44 \\
\hline & 29 & 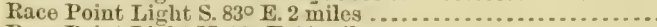 & 30 & 61 & 58 & 44 \\
\hline & 29 & 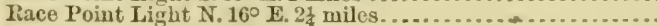 & 27 & 61 & 58 & 44 I.: \\
\hline
\end{tabular}

* See pages 37 and 43. 
DREDGING STATIONS OF U. S. FISH COMMISSION.

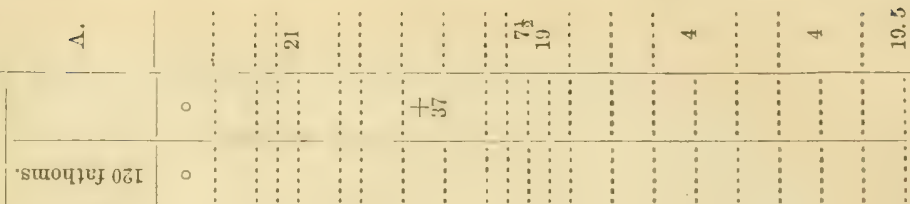

-stuot+ig o[r

"sนтоप⿴囗\zh25 005

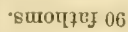

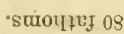

"surorzer 02

'suroqtuy 09

suoपtry 99

'suotruy 09

-

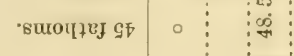

$\approx$

suroquej

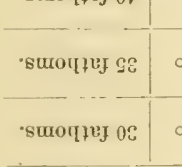

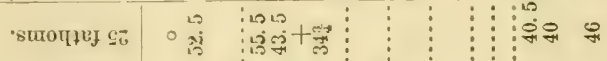

\begin{tabular}{|c|c|c|c|c|c|}
\hline surवारु 06 & 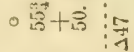 & 突 & テデすું & +9 & $+\infty$ \\
\hline
\end{tabular}

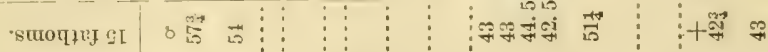

恶

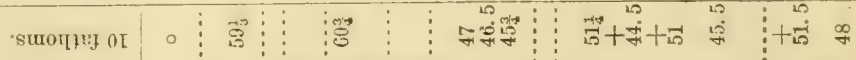

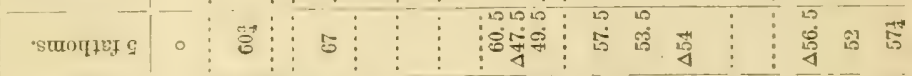

\begin{tabular}{|c|c|c|c|c|c|c|c|c|c|c|c|c|c|}
\hline 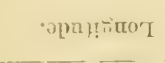 & \begin{tabular}{l|l}
- & $\vdots$ \\
- & $\vdots$
\end{tabular} & $\begin{array}{l}5 \\
\vdots 0 \\
8\end{array}$ & & & $\because$ & 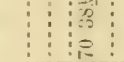 & 㱐 & & & 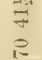 & & & $\vdots$ \\
\hline 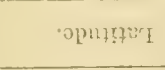 & - & \begin{tabular}{l}
$\vdots$ \\
$\vdots$ \\
$\vdots$ \\
\hdashline
\end{tabular} & & & $\begin{array}{l}0 \\
21\end{array}$ & & $\begin{array}{l}\text { के } \\
\text { के } \\
\text { sy }\end{array}$ & & & 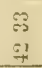 & & & \\
\hline 'xoquma [m!xins' & 0 & 유교몸 & 83 & $\ddot{3}$ & 8 & 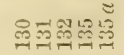 & 武 & 烏 & $\stackrel{20}{7}$ & 吾 & 웍 & 28 & 疍 \\
\hline$\cdot n \times \alpha$ & 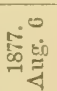 & $\infty 0_{0}^{\infty}$ & ค9? & $?$ & & 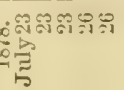 & $\%$ & $\stackrel{-1}{\dot{21}}$ & - & $\curvearrowleft$ & m & $\infty$ & $\infty$ \\
\hline
\end{tabular}


40 REPORT ON COMMSSIONER OF FISII ANI HISIEMIES.

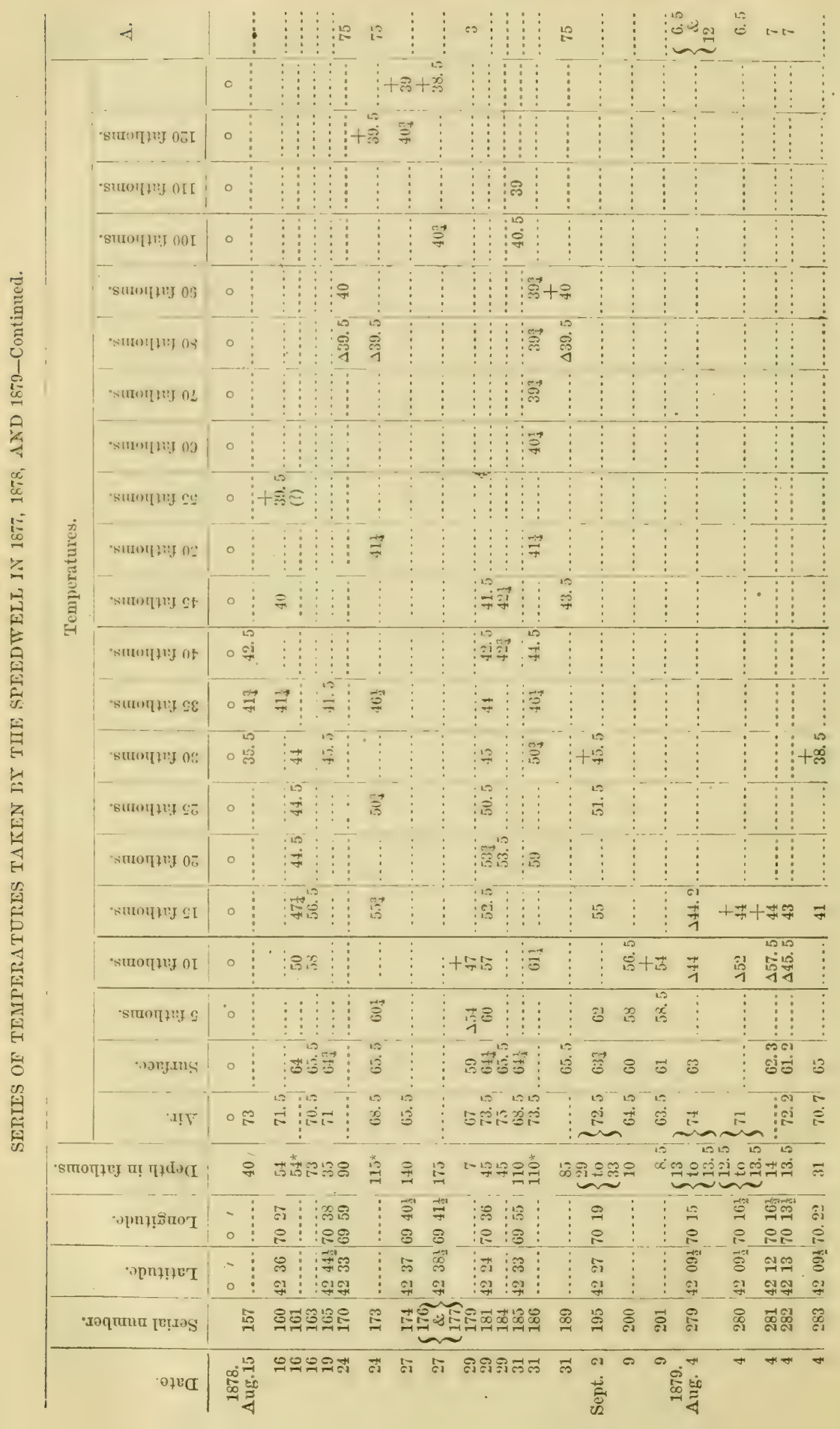




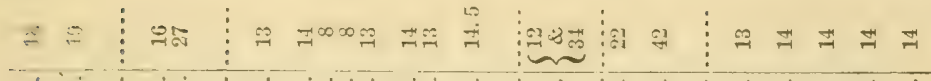

$\vdots+\infty$

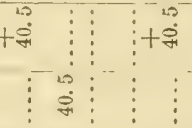

$+\infty$

F

$\infty$

궁

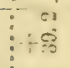

$+\infty \quad+\infty$

F

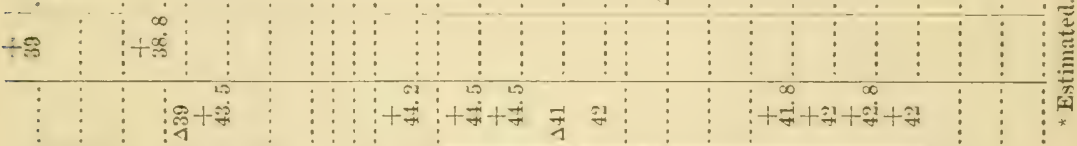

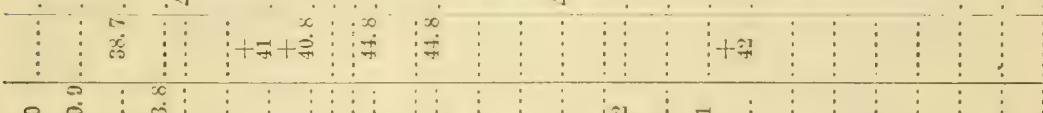

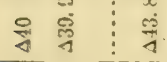

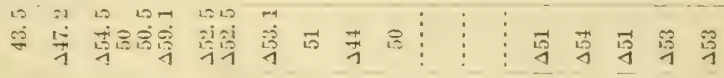

影最

$+10+8$ is

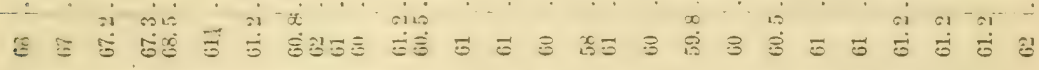
중 औด

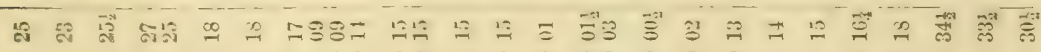

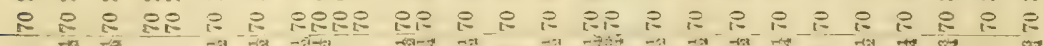

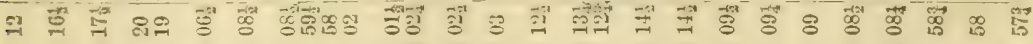

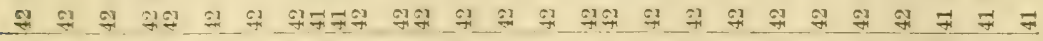
总 话 


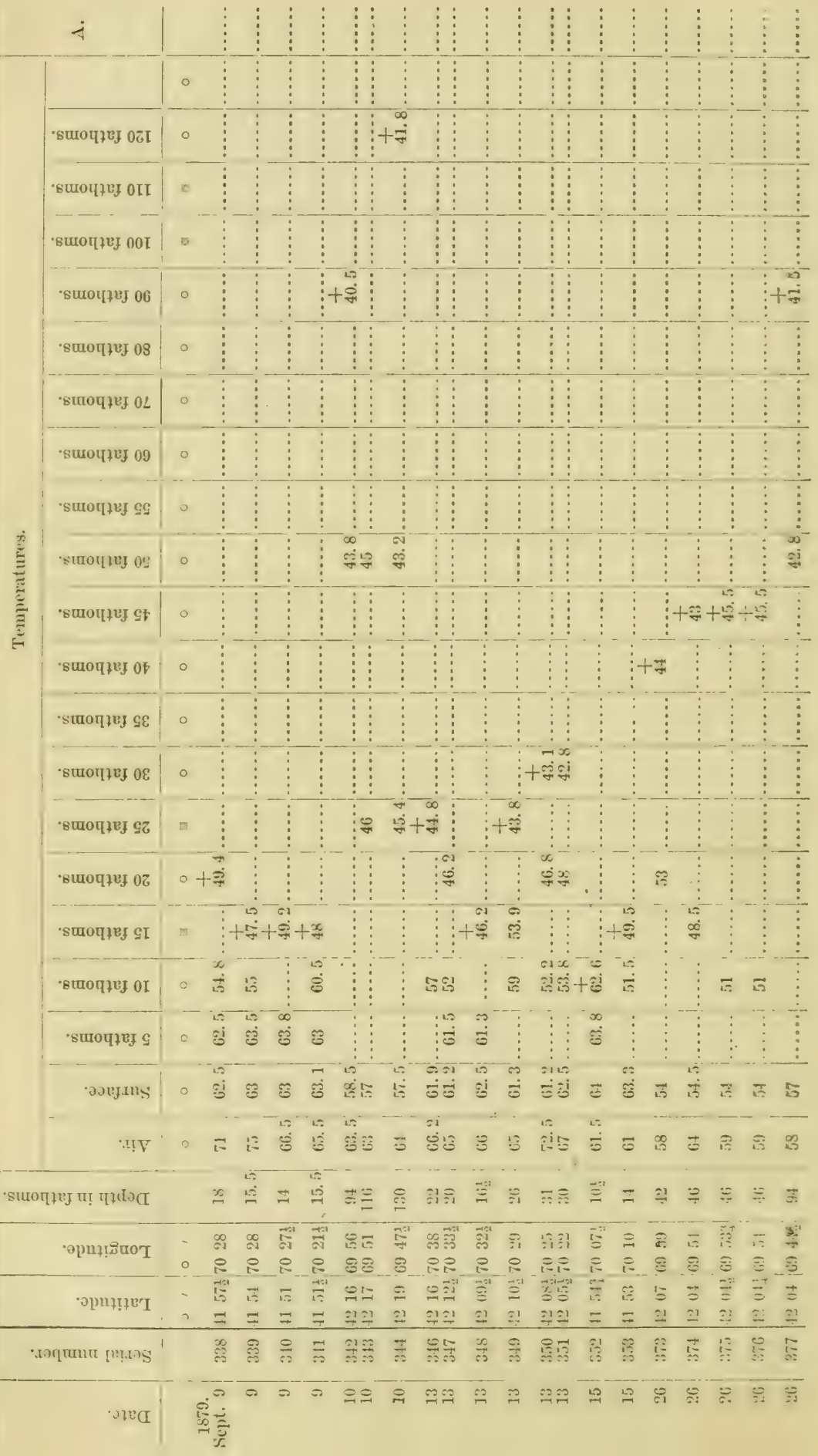




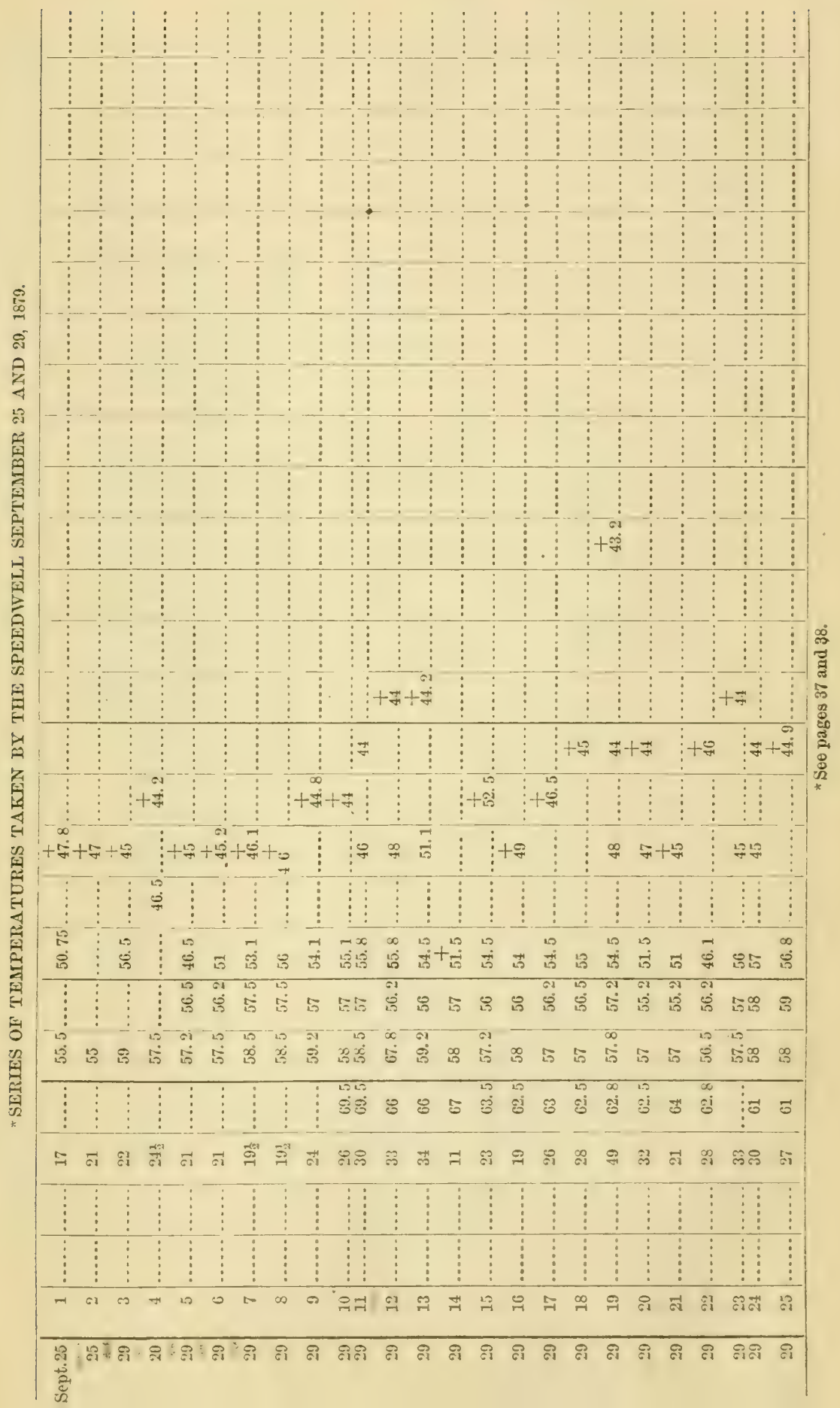













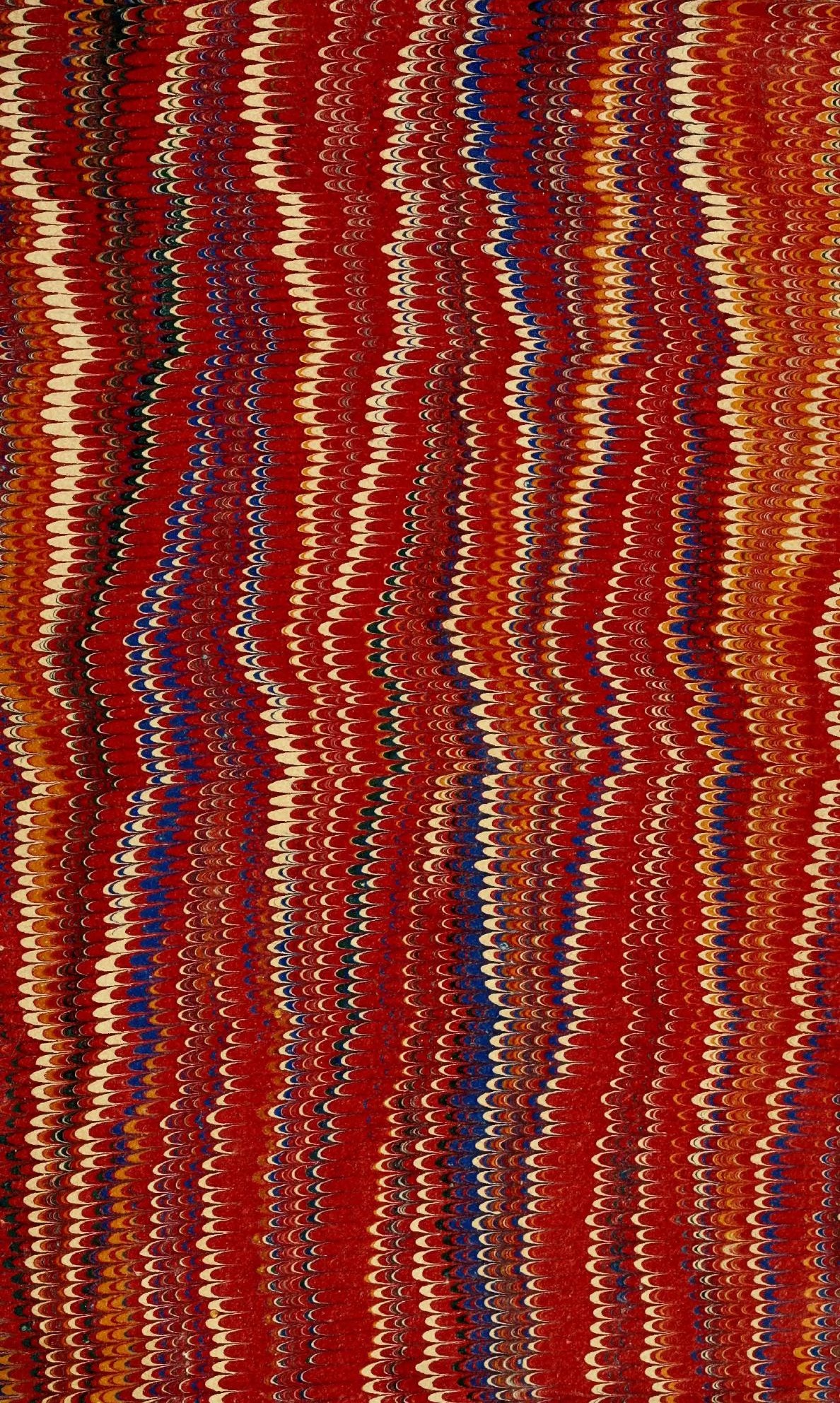


SMITHSONIAN INSTITUTION LIBRARIES

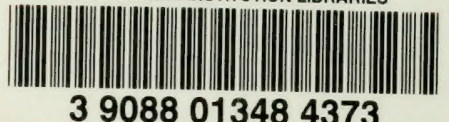

39088013484373 